\title{
SEX-BASED DIFFERENCES IN LIFTING TECHNIQUE UNDER INCREASING LOAD CONDITIONS: A PRINCIPAL COMPONENT ANALYSIS
}

\author{
By \\ PHILLIP SAMPSON SHEPPARD
}

\begin{abstract}
A thesis submitted to the Department of Kinesiology and Health Studies
In conformity with the requirements for

the degree of Master of Science
\end{abstract}

\author{
Queen's University \\ Kingston, Ontario, Canada
}

(September, 2012) 


\begin{abstract}
The objectives of the present study were: 1) to determine if there is a sex-based difference in lifting technique across increasing load conditions; and, 2) to examine the use of body size-adjusted tasks and back strength-adjusted loads in the analysis of lifting technique. Eleven male and 14 female participants $(n=25)$ with no previous history of low back pain participated in the study. Participants completed freestyle, symmetric lifts of a box with handles from the floor to table height for five trials under three load conditions $(10 \%, 20 \%$, and $30 \%$ of their individual maximum isometric back strength). Joint kinematic data for the ankle, knee, hip, and lumbar and thoracic spine were collected using a two-camera Optotrak 3020 system (NDI, Waterloo, ON). Joint angles were calculated using a three-dimensional Euler rotation sequence and PCA was applied to assess differences in lifting technique across the entire waveform. A repeated measures ANOVA with a mixed design revealed no significant effect of sex for any of the PCs. This was contrary to previous research that used discrete points on the lifting curve to analyze sex-based differences but agreed with more recent research using more complex analysis techniques. There was a significant effect of load on lifting technique for six PCs of the lower limb $(\mathrm{p}<0.005)$. However, there was no significant difference in lifting technique for the thoracic and lumbar spine. It was concluded that, when load is standardized to individual back strength characteristics, males and females adopted a similar lifting technique. In addition, as load increases participants used more of a semisquat or squat lifting technique.
\end{abstract}




\section{Acknowledgements}

There are many people I would like to acknowledge who have had a profound impact on this project and my learning over the past two years.

First, I would like to thank my supervisor Dr. Joan Stevenson for all of her guidance and support. I could not have hoped for a better role model. Joan, your work ethic and passion for your students and research are truly inspiring. Your mentorship has allowed me to grow academically, professionally, and personally. I will strive to be as positive and passionate as you in all of my future endeavors.

Second, I would like to thank Dr. Ryan Graham for all of his help and great times over the past two years. Ryan, I wish you the best of luck and happiness as you start your new career and family. Your students are lucky to have such a dedicated and personable professor.

To Dr. Pat Costigan and the rest of the biomechanics lab, thank you for your contributions to this project. Pat was always available and willing to help with MatLab and data processing. I would also like to acknowledge Brett Whitfield for all of his help during long data collection sessions. I am lucky to be surrounded by such kind and intelligent individuals.

Next, I would like to acknowledge my family. To my amazing parents who have provided me with an enormous amount of support in all of my academic and personal endeavors. From an early age you have taught me the dedication to surmount any challenge. Through example you have taught me to always be the best that I can, and to always finish what you start. Most importantly, you have taught me to always be generous and kind. To Steve and Haley, thanks for being there.

Additionally, I would like to thank my friends for always supporting me with all of the goals I have set for myself. I don't know what I would do without you. 
Finally, I would like to thank all of my teachers and coaches for igniting my interest in the human body and learning. Of considerable mention is Dr. Angie Thompson. Her kindness and enthusiasm for teaching and research is what sparked my interest in academics. I hope to be as inspiring as you one day! 


\section{Table of Contents}

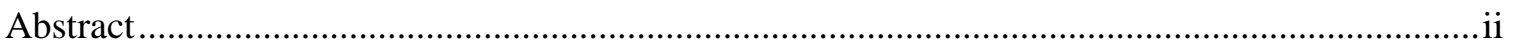

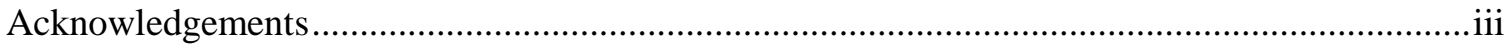

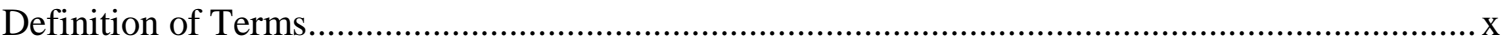

Chapter 1 General Introduction …...................................................................................... 1

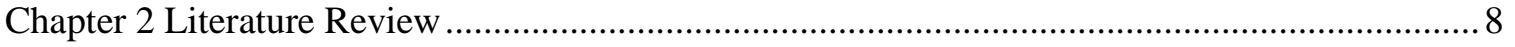

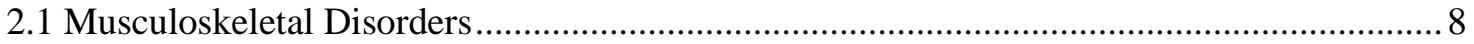

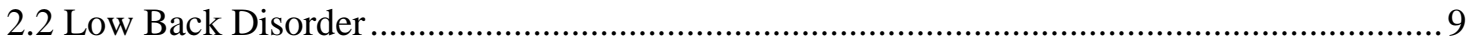

2.3 Anatomical Differences between Males and Females ..................................................... 11

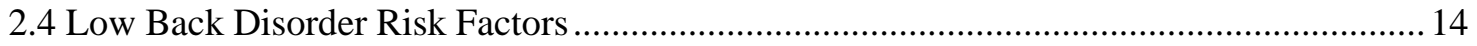

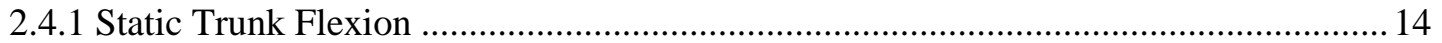

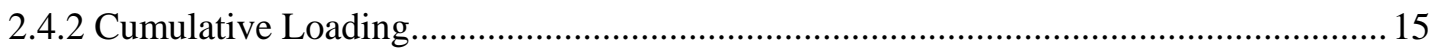

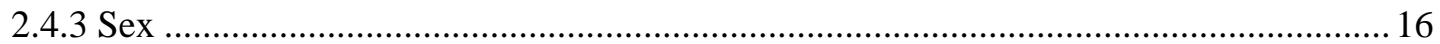

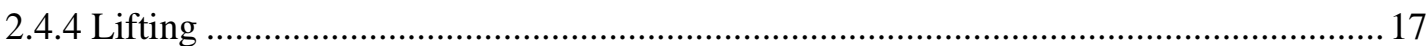

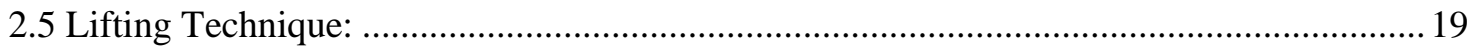

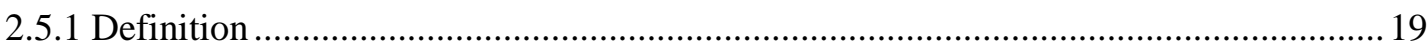

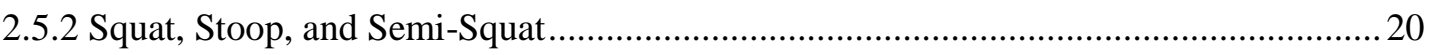

2.6 Sex-Based Differences in Lifting Technique..................................................................26

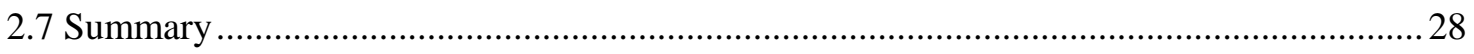

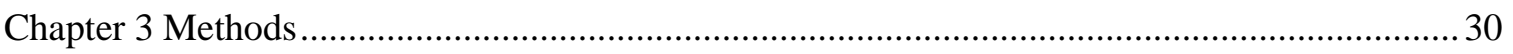

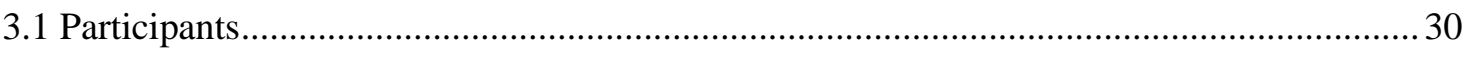

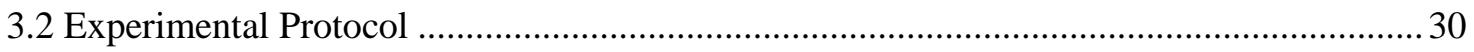

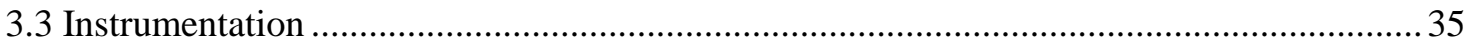

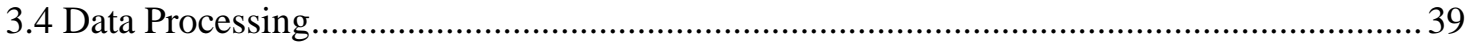

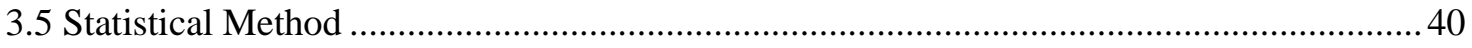

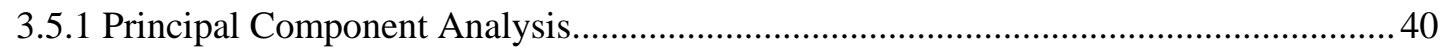

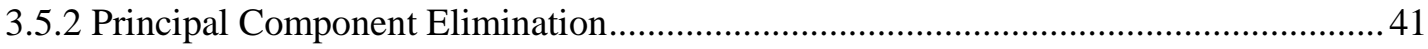

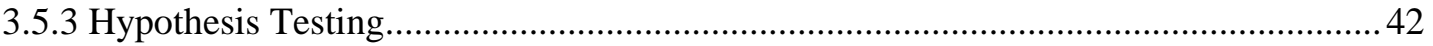

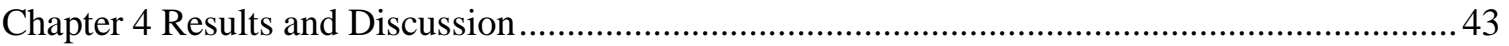

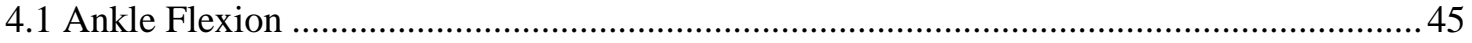

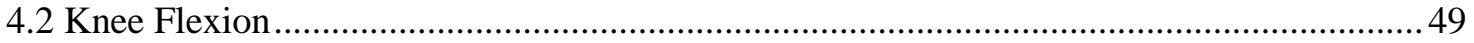




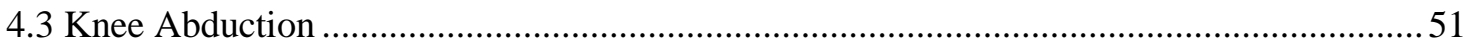

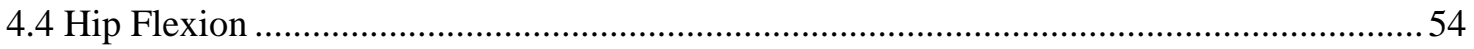

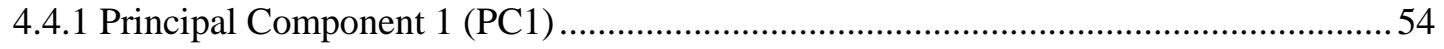

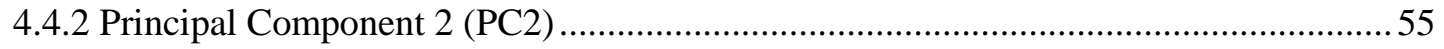

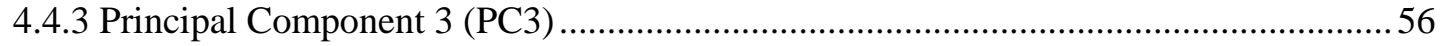

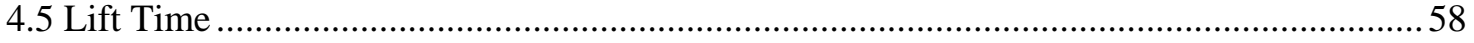

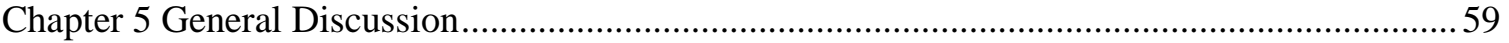

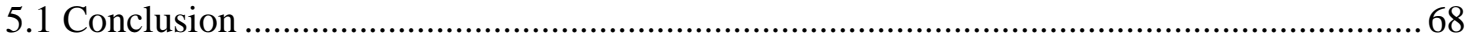

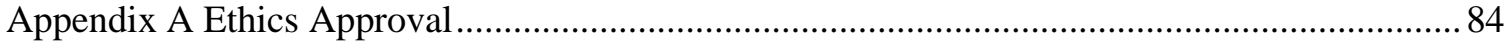

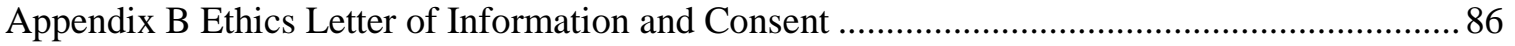

Appendix C Vertebral Column Anatomy and Function ........................................................... 91

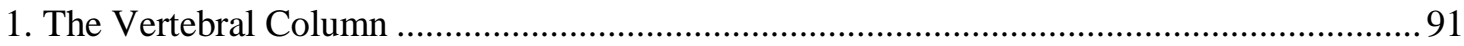

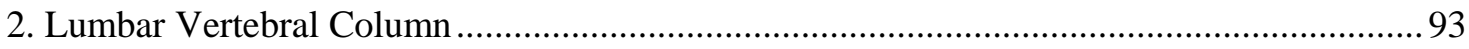

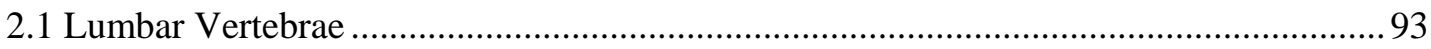

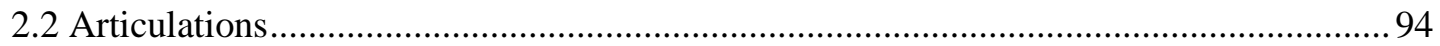

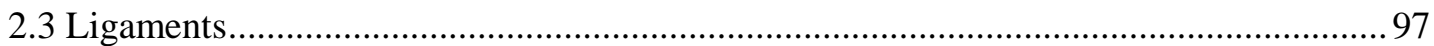

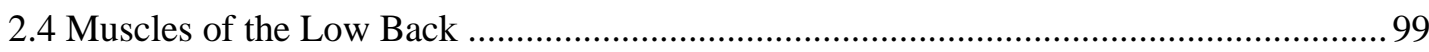

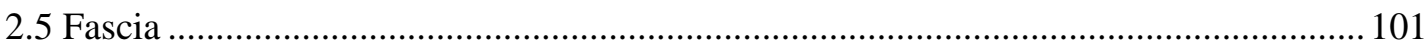

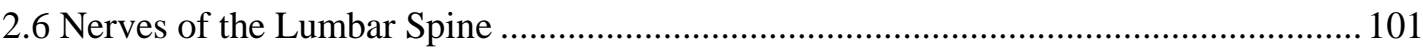

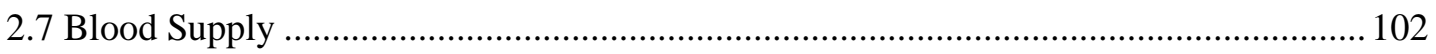

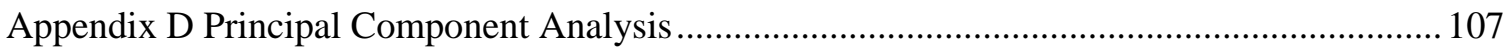

Appendix E Principal Component Elimination ……............................................................ 112

Appendix F Principal Components Retained According to the 90\% Trace Criterion ................. 118

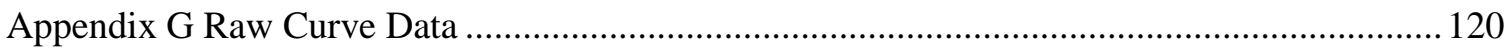




\section{List of Figures}

Figure 1. Participant completing a maximum back strength test against a horizontally-oriented load cell on the modified functional capacity evaluation system.

Figure 2. Experimental set up with a wooden handled box $(36.7 \mathrm{~cm} \times 29.2 \mathrm{~cm}$ x $25.5 \mathrm{~cm})$ loaded to $10 \%, 20 \%$, and $30 \%$ of participant's maximum back strength, and table adjusted to $50 \%$ of participant's height

Figure 3. Participant completing one lift cycle. Participants were required to lift a handled box loaded with $10 \%, 20 \%$, and $30 \%$ of their maximum isometric back strength from a target on the floor to a target on a table (positioned at $50 \%$ of their height) in a freestyle manner.

Figure 4. Participant instrumented with Infrared Emitting Diode (IRED) triads (lateral side of the right foot, shank and thigh, as well as on custom-made fins attached at the levels of S1, T12, and C7), and single IRED markers (lateral malleolus and epicondyle)

Figure 5. Experimental set up with one Optotrak 3020 (NDI, Waterloo, ON) camera oriented perpendicular to the sagittal plane lifting, and one camera oriented slightly behind and on an angle. The orientation of the cameras was chosen to have all makers visible throughout the entire lift.

Figure 6. Box instrumented with two analog switches (handle and bottom of box) to indicate the start and end of the lift.

Figure 7. A) Mean ankle flexion angle during lift for 10\%, 20\%, and 30\% load conditions. Dorsiflexion is in the negative direction whereas plantar flexion is positive. B) Loading vector for ankle flexion principal component 1 (PC1). C) Ankle flexion waveforms for participants who scored high (95th percentile) and low (5th percentile) on PC1 of ankle flexion. Dorsiflexion is negative and plantar flexion is positive.

Figure 8. A) Mean knee flexion angle during lift for 10\%, 20\%, and 30\% load conditions. Knee flexion is positive and knee extension is negative. B) Loading vector for knee flexion principal 
component 1 (PC1). C) Knee flexion waveforms for participants who scored high (95th percentile) and low (5th percentile) on PC1. Knee flexion is positive, whereas extension is negative

Figure 9. A) Mean knee abduction angle during lift for 10\%, 20\%, and 30\% load conditions. Knee abduction (knees away from midline) is positive, and knee adduction (knees towards midline) is negative B) Loading vector for knee abduction principal component 1 (PC1). C) Knee abduction waveforms for participants who scored high (95th percentile) and low (5th percentile) on PC1. Knee abduction is positive, whereas knee adduction is negative. .53

Figure 10. A) Mean hip flexion angle during the lift for 10\%, 20\%, and 30\% load conditions. Hip flexion is positive, and hip extension is negative B) Loading vector for hip flexion principal component 1 (PC1). C) Hip flexion waveforms for participants who scored high (95th percentile) and low (5th percentile) on PC1. Hip flexion is positive, whereas hip extension is negative D) Loading vector for hip flexion PC2. E) Hip flexion waveforms for participants who scored high and low on PC2 using principal component elimination (PCE). Hip flexion is positive and extension is negative F) Loading vector for hip flexion PC3. G) Hip flexion waveforms for participants who scored high and low on PC3 using PCE. Flexion is positive and extension is negative

Figure 11. The orientation of facet joints in the cervical, thoracic, and lumbar vertebrae (Drake, 2010)

Figure 12. Flow chart describing principal component elimination (PCE). 113 


\section{List of Tables}

Table 1. Descriptive statistics for male and female participants

Table 2. Mean maximum isometric back strength and loads lifted by males and females

Table 3. Means, standard deviations, and p-values for principal component scores retained in accordance with a $90 \%$ trace criterion that demonstrated significance. A $p$ value of $p=0.005$ was used to determine significance and are bolded in this table 44

Table 4. Mean maximum (degrees), minimum (degrees), range (degrees), and start position (degrees) for flexion/extension angles of the ankle, knee, hip, and lumbar, and thoracic spine. None of the discrete variables were significant across load conditions based on a repeated measures analysis of variance with a significance level of $\mathrm{p}<0.005)$.

Table 5. Mean lift time (seconds) between males and females for 10\%, 20\%, and 30\% load conditions. No significant differences in time were found between males and females based on an ideendant samples t-test $(\mathrm{p}<0.05)$

Table 6. Mean lift time (seconds) for all participants for 10\%, 20\%, and 30\% load conditions. There was a significant difference between load conditions as determined by an independent samples t-test $(\mathrm{p}<0.05)$

Table 7. Means, standard deviations, and $\mathrm{p}$ values for males and females for all principal components (PCs) retained according to the $90 \%$ trace criterion. A p value of $p=0.005$ represented a significant difference. 


\section{Definition of Terms}

3D

ASIS

Quadriceps Angle

(Q Angle)

ANOVA

$\mathrm{C}_{7}$

EMG

$\mathrm{Hz}$

IRED

LBD

$\mathbf{L}_{4} / \mathbf{L}_{5}$

$\mathbf{L}_{5} / \mathbf{S}_{1}$

MSD

N

PC

PCA

PCE

SD

$\mathrm{S}_{1}$
Three-Dimensional

Anterior Superior Iliac Spine

The angle formed by a line drawn from the anterior superior iliac

spine (ASIS) to the center of the patella, and a line drawn through the center of the patella and the tibial tuberosity.

Analysis of Variance

Cervical Vertebra 7

Electromyography

Hertz

Infrared Emitting Diode

Low Back Disability

Lumbar Vertebrae 4/5

Lumbosacral Sulcus (Lumbar Vertebra 5 and Sacral Vertebra 1)

Musculoskeletal Disorder

Newton

Principal Component

Principal Component Analysis

Principal Component Elimination

Standard Deviation

Sacral Vertebra 1 


\section{Chapter 1}

\section{General Introduction}

Biomechanics and ergonomics research focuses extensively on decreasing the risks and prevalence of musculoskeletal disorders (MSDs). MSDs describe conditions that involve damage to various structures such as muscles, tendons, ligaments, joint, nerves, and supporting blood vessels (Lemasters et al., 1998; Punnett \& Wegman, 2004; Punnett et al., 2005). MSDs are the most common cause of long-term disability and pain (Brooks, 2006; Kumar, 2001; LeBlanc \& LeBlanc, 2010) and result in major financial burden on a national and individual level (Coyte, Asche, Croxford, \& Chan, 1998). Of all the MSDs, low back disorder (LBD) is consistently the most prevalent and economically taxing (Brooks, 2006; Kumar, 2001; LeBlanc \& LeBlanc, 2010; Punnett \& Wegman, 2004).

Low back disorder (LBD) is pain localized below the line of the twelfth rib and above the gluteal folds (Anderson, 1986; Anderson \& Chaffin, 1986). LBD encompasses a number of conditions and injuries including: strains, sprains, fractures, disc injuries, degenerative disorders and rheumatic diseases (Marras, 2008). The majority of individuals affected by LBD are between the ages of 25 and 64 years (Nelson et al., 2005). This age group corresponds with the working population and agrees with the fact that $37 \%$ of all LBD is attributed to occupational factors (Nelson et al., 2005; Punnett et al., 2005). Of all the MSDs, LBD is the most common reason patients seek medical or alternative health care (Feuerstein, Marcus, \& Huang, 2004). The cost of medical care contributes to the large financial burden of LBD. However, indirect costs, such as: days off work, decreased productivity, and insurance claims are also major contributors. The annual cost of LBD in Canada is estimated to be $\$ 16.4$ billion (Badley, Rasooly, \& Webster, 
1994; Coyte et al., 1998). In the United States, the estimated annual cost of LBD is between $\$ 100$ and $\$ 200$ billion (Katz, 2006).

There are a number of occupational risk factors associated with the development of LBD. The most studied risk factors include: lifting, forward bending, static postures, rapid work pace, cumulative loading, insufficient recovery time, asymmetric body postures, vibration, sex, low temperatures, and psychosocial factors (Anderson \& Chaffin, 1986; Kumar, 1990; Kumar, 2001; Norman et al., 1998; Punnett et al., 2005). Of all the factors mentioned, lifting has been regarded as the greatest risk to developing LBD (Hsiang, Brogmus, \& Courtney, 1997). As a result, engineering controls (Graham, Sadler, \& Stevenson, 2011; Lotz, Agnew, Godwin, \& Stevenson, 2009) and administrative controls (Hsiang et al., 1997) have been implemented to reduce the amount of stress on the low back during lifting. Education on proper lifting technique is the most economically feasible and, therefore, is the most frequently used. However, a recent systematic review found that there is no evidence to support the use of standardized training in working techniques for preventing back pain (Martimo et al., 2008). When training is tailored to the individual employee, education on proper lifting and body mechanics has proven to be effective (Albaladejo et al., 2010; Andrade, Araujo, \& Vilar, 2008; Cheng \& Chan, 2009). Therefore, it is important to understand individual preferences in lifting in order to develop effective workplace training programs.

In terms of sex, earlier studies found that males have a higher prevalence of LBD than females (Punnett et al., 2005). Recently, however, studies are reporting that females have a higher prevalence of LBD than males (Freburger et al., 2009). The rising prevalence of LBD in females may be partially attributed to the fact that more females are obtaining employment in physically demanding occupations than in the past. However, there are still more males than females in jobs 
that are physically demanding (Statistics Canada, 2011). Therefore, other factors likely contribute to the findings.

Anatomical differences might partially explain the differences in workplace trends. Males and females differ in terms of body height, weight, size, and shape. After 20 years of age, males are, on average, $14 \mathrm{~cm}$ taller and $12 \mathrm{~kg}$ heavier than females (taken from Clinical Growth Charts, 2006 as cited by Sizer \& James, 2008). However, there are more than simply differences in the size of various structures. Males and females differ in tissue composition, and skeletal alignment. The many anatomical differences can greatly influence movement patterns and risk of injury.

Sex-related differences are apparent throughout the spine and extremities. Beginning with the cervical spine, females have cervical vertebrae with smaller lamina height, width, thickness, and angulation (Xu, Burgar, Ebraheim, \& Yeasting, 1999). It has also been shown that females have smaller pedicular widths, lengths, and transverse angle from $\mathrm{C} 3$ to $\mathrm{C} 7$ compared to males (Rezcallah, Xu, \& Ebraheim, 2001). Females have less articular cartilage in the cervical facet joints than males (Yoganandan \& Knowles, 2003). This causes females to have more translation at those joints than males, which may increase their risk of injury (Siegmund, Sanderson, \& Myers, 2003). It has also been found that females have lower endplate fracture loads in the cervical vertebrae than males due to a lower tissue tolerance (Truumees, Demetropoulos, \& Yang, 2003). In the thoracic spine, the main differences between males and females are seen in postural alignment. Mainly, females have a greater kyphosis than males (Ryan \& Fried, 1997).

The lumbar vertebrae present a number of differences between males and females as well. In terms of posture, females tend to have a greater lumbar spine lordosis than males (Norton, Sahrmann, \& Van Dillen, 2004). Males on the other hand, have greater bone density and facet size in the lumbar vertebrae compared to females (Ebbesen, Thomsen, \& Beck-Nielsen, 
1999). As a result of the reduced bone density, and bone size, females have a decreased compressive load tolerance in comparison to males (Cheng, Yang, \& Huey-Jen, 2001). The muscles of the lumbar spine differ between sexes. Females have decreased Type II fiber diameter in comparison to men (Ng, Richardson, \& Kippers, 1998). This causes females to have increased muscular endurance of the lumbar muscle groups, whereas males have increased strength $(\mathrm{Ng}$ et al., 1998). In addition to structural differences, males appear to have greater stiffness and decreased segmental motion in the lumbar spine compared to females (Brown, Holmes, \& Heiner, 2002). Therefore, females are more susceptible to developing clinical lumbar instability (Brown et al., 2002).

The structure and alignment of the lower extremities have been shown to differ between males and females. Females have a larger pelvis than males to accommodate for childbirth. This includes a larger pelvic inlet and outlet and more widely set hips (Smith \& Smith, 2002). Females appear to have a reduced acetabular depth and femoral neck strength than males, which places them at a greater risk for hip fractures. All of the differences at the pelvis and hip result in femoral anteroversion, increased quadriceps angle ( $\mathrm{Q}$ angle), genu valgum, external tibial torsion, and over-pronation at the ankle joints in females (Smith \& Smith, 2002). Females also have increased knee joint laxity (Trimble, Bishop, \& Buckley, 2002). The differences described in the lower extremity may influence movement patterns when lifting.

Further differences between sexes exist in tissue composition. Males have greater bone mineral density, peak bone mass, cortical thickness, bone turnover, and bone strength than females (Henry \& Eastell, 2000; Nordin \& Frankel, 2001). Males also have greater articular cartilage thickness and surface area compared to females. Given the different anthropometric and strength characteristics between males and females, it is possible that the workstations and lifting 
strategies taught to workers, which are traditionally designed for males, are not optimal for female workers.

The majority of studies examining sex-based differences in spinal loads during lifting have observed differences between males and females. Bejjani, Gross and Pugh (1984) used a two-dimensional static model and found that back and knee shear forces were greater in females, and back compression at $\mathrm{L}_{4} / \mathrm{L}_{5}$ was greater in males during a symmetrical lift. Stevenson, Greenhorn, Bryant, Deakin, and Smith (1996) found significant differences between males and females in terms of timing, displacement, velocity, acceleration, force, and power using an incremental lifting machine (ILM) test. When participants were asked to lift boxes onto a high shelf, men and women frequently chose different lifting styles to lift the same load mass (Stevenson et al., 1990). Marras, Davis, and Jorgensen (2002) used an electromyography-assisted model to examine spine loading and musculoskeletal control strategies. From their study it was discovered that absolute compression was higher for males and relative compression and anteriorposterior shear forces on the low back were greater for females. The differences were attributed to muscle co-activation patterns and compensatory kinematic differences between males and females. In 2003, Marras, Davis, and Jorgensen further investigated differences in lifting technique between sexes by having males and females exposed to similar workplace demands. This time, the findings showed that males experienced greater compression and anterior-posterior shear forces in the low back compared to females. However, sex only accounted for a small percentage of the variability; task asymmetry and load weight had a greater impact on spinal compression than sex.

Lindbeck and Kjellberg (2001) directly examined sex-based differences in lifting technique. The lifting protocol involved participants lifting a handled box loaded with $12.8 \mathrm{~kg}$ for 
males and $8.7 \mathrm{~kg}$ for females, from the floor to a table at waist height. Kinematic differences were found between sexes. However, the loads used were standardized for males and females and did not take into account individual strength characteristics. In addition, the biomechanical model used represented the trunk as one segment and may not have accurately represented the spinal curvature of the participants.

A recent study examined differences in lifting technique between males and females by having participants continuously lift a handled box from the floor to a table positioned at $50 \%$ of the participants' height (Sadler, Graham, \& Stevenson, 2011). Lifting technique was examined for $0 \%$ and $10 \%$ of each individual's maximum isometric back strength using principal component analysis (PCA). No significant differences were observed in terms of sex, but there was a significant effect of load. Based on these findings, Sadler et al. (2011) suggested that, when comparing men's and women's lifting technique, load should be standardized to individual back strength and analysis should involve techniques that allow examination of the entire lifting waveform rather than simply discrete points. In conclusion, the researchers found that under light load conditions, when loads were standardized to individual back strength, males and females adopt a similar lifting strategy (Sadler et al., 2011). However, they also recommended that more research was required to examine whether the same findings appeared with heavier loads.

The goal of this study was to fill in the gap between commonly assumed sex differences based on anatomical differences and lifting technique differences. Thus, the objectives of this study were: 1) to determine if there is a sex-based difference in lifting technique across increasing load conditions; and, 2) to examine the use of body size-adjusted tasks and back strength-adjusted loads in the analysis of lifting technique. Based on the study by Sadler et al. (2011) it was hypothesized that: 1) males and females would use a similar lifting technique under light load 
conditions, and 2) differences in lifting technique between sexes would become apparent with increasing load. This body of work will contribute to the field of biomechanics and ergonomics in terms of findings and methodology. First, greater knowledge will be gained in terms of how males and females lift heavier loads. These results will aid in the development of safe lifting guidelines in occupational settings with the goal of reducing the LBD risk factors associated with lifting. Second, the methodology will involve the use of body size-adjusted tasks and back strength-adjusted loads which create a better methodology to examine sex differences in lifting technique. If the results reveal no sex differences, then it is either the task constraints, or load lifted that are responsible for creating the sex difference in lifting technique found in previous research. Third, the use of principal components analysis, along with a principal component elimination strategy, will serve to examine the entire lifting waveform rather than peaks and timing of peaks. 


\section{Chapter 2}

\section{Literature Review}

\subsection{Musculoskeletal Disorders}

Musculoskeletal disorders (MSDs) incorporate a number of acute and chronic conditions that are linked by their association with pain and impaired physical function, and often result in a decreased quality of life for the individuals affected (Coyte et al., 1998). MSDs involve damage to the muscles, tendons, ligaments, joints, peripheral nerves, and supporting blood vessels (Lemasters et al., 1998; Punnett \& Wegman, 2004; Punnett et al., 2005). The conditions include the inflammation of various structures (tenosynovitis, epicondylitis, bursitis, etc.), nerve compression disorders (carpal tunnel syndrome, sciatica, etc.), osteoarthritis, rheumatoid arthritis, osteoporosis, joint pain and stiffness, as well as neck and low back pain (Brooks, 2006; Coyte et al., 1998; Dagenais, Caro, \& Haldeman, 2008; LeBlanc \& LeBlanc, 2010; Lemasters et al., 1998; Punnett et al., 2005; Woolf \& Pfleger, 2003).

Globally, MSDs are the most common cause of long-term pain and disability (Bernard, 1997; Brooks, 2006; Kumar, 2001; LeBlanc \& LeBlanc, 2010; Woolf \& Pfleger, 2003) and the single most common cause of work-related illness (Bernard, 1997; Punnett \& Wegman, 2004; Punnett et al., 2005). MSDs affect up to $20 \%$ of adults worldwide (Woolf \& Pfleger, 2003). At any one point in time, $30 \%$ of adults from the United States are affected by MSDs (Woolf \& Pfleger, 2003). In Europe and Australia, MSDs have been reported to affect $20 \%$ and $22 \%$ of adults, respectively (Brooks, 2006). The Ontario Health Survey reported that MSDs caused 40\% of all chronic conditions, $54 \%$ of all long-term disability, and $24 \%$ of all restricted activity days 
(Badley et al., 1994). The extremely high prevalence of MSDs results in a huge economic burden on a global and local scale. In 1994, the total cost of musculoskeletal disorders in Canada was $\$ 25.6$ billion, or $3.4 \%$ of the gross domestic product (GDP) (Coyte et al., 1998). In the United States the economic cost of MSDs in 1992 amounted to $\$ 149.4$ billion or $2.5 \%$ of the GDP (Coyte et al., 1998). Of all the MSDs, low back disorder is consistently the most prevalent and costly (Brooks, 2006; Kumar, 2001; LeBlanc \& LeBlanc, 2010; Punnett \& Wegman, 2004).

\subsection{Low Back Disorder}

Low back disorder (LBD) is defined as pain localized below the line of the twelfth rib and above the inferior gluteal folds (Anderson, 1986). It encompasses a number of conditions from injuries such as strains, sprains, fractures, and disc problems, to degenerative and rheumatic disorders (Marras, 2008). LBD is the most prevalent MSD in the world (Badley et al., 1994; Brooks, 2006; Coyte et al., 1998; Dagenais et al., 2008; Freburger et al., 2009; Hoy et al., 2010; Kumar, 2001; LeBlanc \& LeBlanc, 2010; Marras, 2008; Punnett \& Wegman, 2004; Punnett et al., 2005; Woolf \& Pfleger, 2003) with the majority of cases (90\%) being idiopathic (Woolf \& Pfleger, 2003). LBD is such a widespread problem that it has been identified by the Pan American Health Organization as one of the top three occupational health problems to be targeted for surveillance by the World Health Organization (WHO) (Choi, Tennassee, \& Eijkemans, 2001). LBD is estimated to affect $4-33 \%$ of the population at any given time (Papageorgiou et al., 1996; van Tulder, Koes, \& Bombardier, 2002; Woolf \& Pfleger, 2003), and up to 85\% of individuals over their lifetime (Freburger et al., 2009; Papageorgiou et al., 1996; van Tulder et al., 2002; Walker, 2000; Woolf \& Pfleger, 2003) with the majority of people being affected between 
the ages of 25 and 64 years (Nelson et al., 2005). The specific age group affected can be explained by the fact that $37 \%$ of LBD has been attributed to occupational factors (Nelson et al., 2005; Punnett et al., 2005). In Canada LBD accounts for the greatest (27\%) of all occupational MSDs (Kumar, 2001), this trend is also apparent in Europe, where LBD constitutes $24 \%$ of all MSDs (Brooks, 2006). Of all the MSDs, LBD is currently the most common reason patients seek medical, chiropractic, and alternative medical care, and a major reason patients obtain care from physical therapists (Feuerstein et al., 2004; Kessler et al., 2001; Martin, Deyo, \& Mirza, 2008). Some studies have also shown that the prevalence of LBD is increasing and, as a result, there has been a significant increase in the number of people seeking care from medical professionals for treatment (Freburger et al., 2009; Kessler et al., 2001).

Low back disorder results in major financial burden due to the cost of medical care, days off work, productivity loss, and insurance claims (Hoy et al., 2010; Woolf \& Pfleger, 2003). The annual cost of LBD in Canada is estimated to be $\$ 16.4$ billion (Badley et al., 1994; Coyte et al., 1998 ) and in the United States, the annual cost of LBD is suggested to be between $\$ 100$ and $\$ 200$ billion (Katz, 2006). The cost is exorbitant and research suggests that it is increasing (Dagenais et al., 2008; Freburger et al., 2009). For example, in the United States, the mean medical cost for individuals with LBD in 1997 was \$4,695. Eight years later, in 2005 the mean cost for individuals with LBD jumped to $\$ 6,096$ (Martin et al., 2008). These values represent a $65 \%$ increase (adjusted for inflations) in total estimated expenditure for people with LBD, a number that far exceeds the increase in total medical expenditure for individuals without LBD (Martin et al., 2008).

In terms of sex, earlier studies found that males have higher prevalence of LBD than females (Punnett et al., 2005). Recently, however, studies are reporting that females have a higher 
prevalence of LBD than males (Freburger et al., 2009). The rising prevalence of LBD in females may be partially attributed to the fact that more females are obtaining employment in physically demanding occupations than in the past. However, there remains to be more males than females in jobs that are physically demanding (Statistics Canada, 2011). Therefore, other factors such as anatomical differences between sexes likely contribute to the rising prevalence of LBD in females.

\subsection{Anatomical Differences between Males and Females}

A number of anatomical and physiological differences exist between males and females. The differences in musculoskeletal anatomy are explained by sexual dimorphism, which refers to the physical differences in secondary sexual characteristics between males and females as a result of sexual maturation (Sizer \& James, 2008). Differences exist between males and females in terms of body height, weight, shape and size. For instance after 20 years of age, males are, on average, $14 \mathrm{~cm}$ taller and $12 \mathrm{~kg}$ heavier than females (taken from Clinical Growth Charts, 2006 as cited by Sizer \& James, 2008). However, differences also exist in the size of various structures and the alignment of the extremities. The combination of the many small differences can have a profound impact on the movement patterns of males and females and their predisposition to injury.

In order to understand the spinal movement patterns of males and females, it is important to take into account their specific structural differences. Sex-related differences in the cervical spine are evident in vertebral structure. Females have been found to contain cervical vertebrae with smaller lamina height, width, thickness, and angulation from $\mathrm{C} 2$ to $\mathrm{C} 5 \mathrm{Xu}$, Burgar, 
Ebraheim \& Yeasting, 1999). In addition, females have been shown to have smaller pedicular widths, lengths, and transverse angles at from C3 to C7 compared to males (Rezcallah et al., 2001). Males appear to have a smaller vertebral canal in proportion to their overall axial skeleton compared to females (Hukuda \& Kojima, 2002). The cervical facet joints are also different between males and females. Siegmund, Sanderson, \& Myers (2003) found that the cervical facet joints in females have more translation than males. The reasoning behind the greater translation may be due to the fact that the facet articular surfaces in females have less cartilage than males (Yoganandan \& Knowles, 2003). This may cause females to be at a greater risk of injury than males, as excessive segmental translation has been shown to be a potential cause of injury (Siegmund et al., 2003) . It has also been found that female cervical vertebrae have a lower tissue tolerance than males. Truumess, Demetropoulos, and Yang (2003) examined loading response of cartilaginous endplates in cadaveric cervical discs and found that females had significantly lower endplate fracture loads than males when exposed to compression. The primary sex-based differences observed in the thoracic spine are centered on postural alignment. Mainly, females have been shown to have greater kyphosis than males (Ryan \& Fried, 1997). Females are also more likely to develop scoliosis than males.

The lumbar spine region presents a number of anatomical differences between males and females. Males have been shown to have a greater bone density in the lumbar vertebrae compared to females (Ebbesen et al., 1999). This results in a decreased compressive load tolerance for females, a problem that becomes worse as women age, especially after menopause (Cheng et al., 2001). Masharawi and colleagues (2004) examined vertebrae from 240 adult human spines and found that facet size was greater in males than females, regardless of the vertebrae measured. 
Sex-based differences have also been found in terms of posture. More specifically, females tend to have greater lumbar spine lordosis than males (Norton et al., 2004).

Apart from bone structure and vertebral alignment, males and females differ in lumbar muscle cross-sectional area and muscle composition. Ng, Richardson, and Kippers (1998) found that females have decreased Type II fiber diameter compared to men. As a result, females have increased muscular endurance of the lumbar muscle groups, whereas males have increased strength (Ng et al., 1998). In addition to muscle fiber composition, males appear to have greater stiffness and decreased segmental motion in the lumbar spine compared to females (Brown et al., 2002). Therefore, females are more likely to develop clinical lumbar instability (Brown et al., 2002).

The pelvis and lower extremities present some sex-based differences that can drastically influence movement patterns. The pelvis is larger in females than males. This includes a larger inlet and outlet to allow childbirth, and more widely set hips (Smith \& Smith, 2002). The larger pelvis results in femoral anteroversion, increased quadriceps angle ( $\mathrm{Q}$ angle), genus valgum, and external tibial torsion (Smith \& Smith, 2002). The Q angle is the angle formed by a line drawn from the anterior superior iliac spine to the center of the patella, and a line drawn through the center of the patella and the tibial tuberosity (Sizer \& James, 2008; Smith \& Smith, 2002). The larger Q angle in females often causes genu valgum (knock knee) in which the individual, standing in neutral position has the knees together and a wide separation at the ankles (Smith \& Smith, 2002). This often results in over-pronation of the ankle joints (Smith \& Smith, 2002). At the hips, females appear to have a reduced acetabular depth and femoral head width (Wang, Brede, \& Lange, 2004). Females also appear to have decreased femoral neck strength than males, 
which places them at a greater risk for hip fracture (Beck, Ruff, \& Scott, 1992). Moving distally, females have increased knee joint laxity as well (Trimble et al., 2002).

In addition to differences in anatomical geometry, there are a number of musculoskeletal differences between males and females. Males have been reported to have greater bone mineral density (Henry \& Eastell, 2000), peak bone mass (Nordin \& Frankel, 2001), cortical thickness, bone turnover (Henry \& Eastell, 2000), and bone strength (Nordin \& Frankel, 2001) than females. Males also have greater articular cartilage thickness and surface area compared to females (Nordin \& Frankel, 2001). As a result of the anatomical differences, females have a higher prevalence of injury and likely have different movement patterns and lifting techniques.

\subsection{Low Back Disorder Risk Factors}

A number of risk factors have been identified as precursors to LBD. The occupational risk factors most cited for LBD include forward bending and static holding (Punnett et al., 2005), rapid work pace and cumulative loading (Anderson, 1986; Kumar, 1990; Kumar, 2001; Norman et al., 1998), insufficient recovery time, non-neutral body postures, vibration, and low temperatures (Punnett et al., 2005). Psychosocial factors also appear to play a role in LBD (Davis \& Heaney, 2000; Linton, 2000).

\subsubsection{Static Trunk Flexion}

Previous research has found that static working postures are related to LBD. In 1991, Punnet et al. reviewed back injury cases from an automotive manufacturing plant and found that 
both mild (21-45 from standing) and severe ( $>45^{\circ}$ from standing) amounts of trunk flexion led to an increased chance of developing LBD. They also suggested that the risk of developing LBD increased with exposure to static postures and the duration of exposure (Punnett, Fine, Keyserling, Herrin, \& Chaffin, 1991). This result is supported in studies by Boussenna, Corlett, and Pheasant (1982) and Keyserling, Sudarsan, Martin, and Haig (2005) who found greater amounts of low back discomfort and pain with greater degrees of forward flexion. Static working postures with the trunk in flexion increases the likelihood of developing LBD because the lever arm, due to body weight and load, is increased which, produces an increased moment about the lumbar vertebral column (Giat \& Pike, 1992).

Repetitive exposure to static postures has been shown to have a number of negative consequences such as joint discomfort, as well as pain in the tendons and muscles. Static postures have also been shown to increase the likelihood of developing arthritis, inflammation of tendons, chronic joint degeneration, muscle spasms, and damage to the intervertebral disc of the lumbar vertebrae (Genaidy \& Karwowski, 1993). Static postures can lead to muscle imbalances between the abdominals and back muscles, which can further lead to LBD (Marras, 2008).

\subsubsection{Cumulative Loading}

Cumulative loading has gained much attention in recent years, as it has been found to be a prevalent risk factor for LBD (Kumar, 1990; Kumar, 2001; Norman et al., 1998). Marras and colleagues (2008) state that, given the conditions of modern work (high repetitions and low force), cumulative loading is more likely to be the mechanism of LBD rather than acute events. Sub-maximal lifting over a prolonged period of time causes muscle fatigue which reduces the 
spine's ability to resist compression and shear forces. The spine's tolerance limit decreases with each cycle (Brinkmann, Biggemann, \& Hilweg, 1988) and has been shown to diminish with age (Jager, Luttman, \& Laurig, 1991; Kumar, 1990). For instance, it has been found that within a given sex, a 60-year old was able to withstand two-thirds of the load tolerated by a 20 -year-old (Jager et al., 1991; McGill, 2002). The tissue tolerance has also been shown to be higher for males; females can tolerate compressive forces that are approximately two thirds what males can withstand (Jager et al., 1991). When the tissue tolerance lowers considerably and approaches the tolerance limit, a low back injury can occur. Therefore, it is important that individuals implement proper lifting technique and rest strategies to prevent LBD, even when lifting sub-maximal loads.

\subsubsection{Sex}

Sex has been found to be a risk factor for LBD. The majority of studies have found that males have a higher prevalence of LBD than females (Punnett et al., 2005). In support of this fact, Punnett et al., (2005) found that $41 \%$ of males reported LBD, compared to $32 \%$ of females. They attributed the higher prevalence of LBD in males to higher participation rate in the labor force and in occupations with heavy physical workload, materials handling, and whole body-vibration. Punnett et al. (2005) also noted that the sex difference was more pronounced in the Eastern Mediterranean region, where female's participation in the labor force is quite low. Recently however, an increasing number of studies are reporting that females have a higher prevalence of LBD. A telephone interview designed to assess the influence of occupational factors on LBD was completed on 8664 Danish workers and found that females were at higher risk of developing LBD than males (Xu, Bach, \& Ørhede, 1997). Freburger et al. (2009) conducted a telephone 
survey in 1992 and 2006 and found that the prevalence of LBD in females has increased at a much greater rate than males, to the point where more females are affected than males. Most notably, in the 21-34 year age group, the prevalence of LBD increased $115 \%$ and $320 \%$ from 1992 to 2006 for males and females, respectively (Freburger et al., 2009).

The rising prevalence of LBD in females may be partially attributed to the fact that more females are working in physically demanding occupations than in the past. However, there remains to be more males than females in jobs that are physically demanding (Statistics Canada, 2011). Therefore, other factors likely contribute to the findings. It is possible that the workstations or lifting techniques taught to workers, which were traditionally designed for males, are not suitable for female workers and place them at greater risk of developing LBD. More research is needed to understand the differences in natural lifting strategies of males and females in order to develop safe lifting strategies and workstations.

\subsubsection{Lifting}

Lifting has been regarded as the greatest risk factor for developing LBD (Hsiang et al., 1997). This is due to the fact that during lifting, as with forward flexed postures, the center of gravity of the trunk is anterior to the lumbar region of the spine. This increases the lever arm and creates a large moment about the lower back (Potvin, McGill, \& Norman, 1991). In order to maintain balance, an extension moment is required to counteract the moment produced by the trunk and load being lifted. The extension moment is accomplished by the recruitment of various trunk muscles and hip extensors (Hsiang \& Mcgorry, 1997; White \& Panjabi, 1990), as well as inter-abdominal pressure (Hsiang \& Mcgorry, 1997), and the use of passive structures such as 
thoracolumbar fascia, intervertebral discs, and ligaments (Adams \& Hutton, 1981; Hsiang \& Mcgorry, 1997; McGill \& Norman, 1986; McGill, 2002). It is evident that lifting creates great strain on the active and passive structures of the back, especially since, the muscles of the lumbar spine are at a mechanical disadvantage compared to the trunk and external load due to the short lever arm relative to the spine (Marras, 2008). Therefore, the muscles of the back must produce a force far greater than the force of the load being lifted. In fact, Marras and colleagues (2008) stated that it is not unusual for an internal force to be ten times greater than the external load. In addition, the contraction of various muscles, along with passive tissue activation generates high shear and compressive forces on the spine (Kumar, 1994; Potvin et al., 1991; Potvin, Norman, \& McGill, 1991). Therefore, as a result of the strain on active and passive tissues, as well as the shear and compressive forces on the spine, lifting can be quite hazardous and is a known cause of LBD.

As a result, many approaches have been made to limit workers' exposure to lifting. These include engineering controls (Graham et al., 2011; Lotz et al., 2009) to reduce the load in the hands and the time spent in non-neutral postures, as well as administrative controls (Hsiang et al., 1997). The most frequently used administrative control involves training workers in correct lifting techniques. However, much debate remains as to which technique, if any, produces the least amount of compressive and stress forces on the lumbar vertebrae. 


\subsection{Lifting Technique:}

\subsubsection{Definition}

Lifting is a very complex process involving a number of different mechanisms and decision-making systems (Hsiang et al., 1997). Lifting involves the combination of functional anatomy, as well as involuntary and voluntary control mechanisms (Hsiang et al., 1997; Winters \& Woo, 1990). Lifting technique is defined as an individual method of performing a lift under given task and environmental conditions (Hsiang et al., 1997). Various lifting techniques are distinguished by non-random, repeatable selection of voluntary and involuntary control mechanisms (Hsiang et al., 1997). However, the manner in which lifting technique is described varies among researchers.

Most authors distinguish between lifting techniques by the amount of knee and hip flexion. For instance, the squat lifting technique is referred to a technique where the knees are flexed and the trunk remains straight, whereas during the stoop lifting technique the knees are extended and the trunk is flexed (Burgess-Limerick \& Abernethy, 1997; Lindbeck \& Kjellberg, 2001; van Dieen, Hoozemans, \& Toussaint, 1999). Lifting using a semi-squat technique, therefore, involves a moderate range of flexion at both the knees and trunk (Burgess-Limerick, 2003; Straker, 2003).

Quantitative definitions have also been developed. Straker (2003) described the squat technique as knee flexion approximately $45^{\circ}$ and trunk flexion less than $30^{\circ}$. The stoop technique is suggested to involve knee flexion greater than $135^{\circ}$ with trunk flexion around $90^{\circ}$ when lifting from floor level (Straker, 2003). Toussaint, van Barr, van Langen, de Looze, and van Dieen (1992) found that when using the stoop lift, the knees remain extended (approximately $10^{\circ}$ of 
flexion) and the trunk goes through a large range of flexion (approximately $50^{\circ}$ ). They also stated that the squat lifting technique involves a large flexion of the knees (approximately $105^{\circ}$ ) and a small amount of trunk flexion (approximately $20^{\circ}$ ) (Toussaint et al., 1992). Burgess-Limerick and Abernethy (1997) developed a postural index based on the ratio between knee flexion and the sum of ankle, hip and lumbar flexion. The squat lifting technique corresponds to postural indices close to 1 , the stoop technique corresponds to postural indices close to 0 , and the semi-squat lifting technique corresponds to postural indices close to 0.5 (Burgess-Limerick \& Abernethy, 1997).

\subsubsection{Squat, Stoop, and Semi-Squat}

There is a constant pursuit in biomechanics and ergonomics research to determine, without error, the safest lifting technique in hopes of decreasing the prevalence of LBD. In analyzing lifting technique, it is important to understand why people prefer using a certain method over another and more importantly, which technique (if any) produces the least amount of stress on the lower back. The majority of research has focused on analyzing the difference between the squat (i.e., knees bent and back straight) and stoop (i.e., knees straight and back bent) lifting techniques. There is an increasing number of articles that have examined the use of the semi-squat lifting technique as well.

Most experienced lifters prefer using the stoop lifting technique (Giat \& Pike, 1992; Hagen \& Harms-Ringdahl, 1994; van Dieen et al., 1999). The reasoning behind this preference is that the stoop lifting technique is less physiologically demanding than the squat lifting technique (Giat \& Pike, 1992; Straker, 2003). Hagen and Harms-Ringdahl (1994) examined maximum 
oxygen consumption, heart rate, and ventilation maximum capacities for squat and stoop lifting for 10 male forestry workers. They reported maximum oxygen consumption for stoop lifting to be $14.3 \%$ less than for squat lifting (Hagen \& Harms-Ringdahl, 1994). They also noted that ventilation and heart rate maximum capacities were $18.7 \%$ and $6.5 \%$ less than for squat lifting, respectively (Hagen \& Harms-Ringdahl, 1994). The difference in physiological demand was attributed to the fact that the squat lifting technique requires the recruitment of more muscle mass to complete the lift (Hagen \& Harms-Ringdahl, 1994). A number of other studies have found that the squat lifting technique results in more energy expenditure (Garg \& Herrin, 1979; Straker, 2003; Welbergen, Kemper, Knibbe, Toussaint, \& Clijssen, 1991), resulting in higher perceived exertion and more rapid fatigue development (Kumar, 1984; Straker, 2003; van Dieen et al., 1999) than the stoop lifting technique. However, the stoop lifting technique is not advocated in industrial and home settings because it is thought to put great stress on the low back.

The squat lifting technique is commonly recommended for lifting tasks because it is thought to drastically reduce the likelihood of back injury and LBD (Straker, 2003). However, the research on this subject has produced conflicting findings. Potvin et al. (1991) evaluated the moment and forces on the low back using kinematics and EMG by having 15 male participants lift various loads $(5.8,13.6,21.5$, and $32.4 \mathrm{~kg})$. The peak lumbar moment for stoop lift was estimated to be $5 \%$ greater than for the squat lifts (Potvin et al., 1991). They also found higher shear forces for stoop lifting compared to squat lifting (Potvin et al., 1991). In terms of compression at L4/L5, Potvin et al. (1991) found that the squat lifting technique produced higher values than stoop lifting. However, they noted that the stress on the low back was more influenced by the degree of trunk flexion than lifting technique, and concluded that the risk of injury may be influenced more by the degree of lumbar flexion (Potvin et al., 1991). 
In 1999, an extraordinary study by Wilke, Neef, Caimi, Hoogland, and Claes, examined intradiscal pressure on one volunteer by surgically inserting a pressure transducer into the nucleus pulposus of the L4/L5 disc. The pressure was recorded during a 24 hour period for various lying and sitting positions, as well as during sneezing, laughing, walking, jogging, stair climbing, and most importantly for our purposes, lifting tasks (Wilke, Neef, Caimi, Hoogland, \& Claes, 1999). While lifting a $20 \mathrm{~kg}$ load, it was found that the interdiscal pressure was $2.30 \mathrm{MPa}$ for the stoop lifting technique, and 1.70 MPa for the squat lifting technique (Wilke et al., 1999). The squat lifting technique reduced the pressure in the disc by approximately $25 \%$ compared to stoop lifting. Therefore, from their study, it was concluded that the squat technique should be advocated for lifting tasks.

Also in support of the squat lifting technique, Anderson and Chaffin (1986) examined five lifting techniques and found that ligament strain, disc compression, and strength requirements were less when individuals used a squat lifting technique. Bzrgari, Shirazi-Adl, and Arjmand (2007) evaluated lumbar and torso flexion, pelvic rotation, and hand position in threedimensions while participants performed sagittally symmetric squat and stoop lifts with and without a $180 \mathrm{~N}$ load in the hands. They found that the maximum net external moment at the L5/S1 disc substantially increased as the $180 \mathrm{~N}$ load was carried in the hands and as the lifting was performed using the stoop technique (Bazrgari, Shirazi-Adl, \& Arjmand, 2007). More specifically, they found that the stoop technique increased the net external moment by $28 \%$ compared to the squat technique.

Further support for using the squat lifting technique is provided by the fact that degenerative disc disease is relatively rare in Eastern populations where people perform a flatback squatting posture on a daily basis as compared to Western countries (Fahrni \& Trueman, 
1965). Although the squat lifting technique has been advocated since 1924 (Straker, 2003), there is conflicting evidence in the literature to support concretely the use of the squat lifting technique as opposed to the stoop lifting technique. If fact, even the studies that have found the squat lifting technique to be less stressful on the low back than the stoop technique have reported lumbar moments between the two techniques that are within $5 \%$ of each other (de Looze, Kingma, Thunnissen, Van Wijk, \& Toussaint, 1994; Kumar, 1994; Toussaint et al., 1992; van Dieen, Creemers, Draisma, Toussaint, \& Kingma, 1994).

A number of studies examining the difference between the squat and stoop lifting technique have found that neither technique is less stressful on the low back. Lindbeck and Arborelius (1991) had 10 male participants lift a $12.8 \mathrm{~kg}$ box using the squat and stoop lifting techniques at two different speeds. From their study, they found no significant difference between the two techniques for peak moment and peak compression at $\mathrm{L}_{4} / \mathrm{L}_{5}$. Therefore, they concluded that, based on their study, there is no biomechanical basis for deciding between the two lifting techniques (Lindbeck \& Arborelius, 1991).

A study by de Looze et al. (1994) evaluated peak lumbar moments and forces using the Static Strength Prediction Model (SSPM, University of Michigan) and a dynamic model after they had five male participants repetitively lower and lift an $11 \mathrm{~kg}$ box from knuckle height in upright standing to $0.10 \mathrm{~m}$ above the ground. They found no significant difference between the squat and stoop lifting techniques using either analysis technique. de Looze and colleagues (1998) further tested the difference between squat and stoop lifting techniques by having 8 males lift a $6.7 \mathrm{~kg}$ and $15.7 \mathrm{~kg}$ box from a shelf positioned $0.02 \mathrm{~m}$ above the ground to knuckle height in erect standing at two speeds (normal and fast). Peak L5/S1 torque and compression was significantly 
affected by speed, and weight (de Looze, Dolan, Kingma, \& Baten, 1998). However, there was no significant effect of lifting technique for any of the variables examined (de Looze et al., 1998).

In 1998, Kjellberg, Lindbeck and Hagberg examined the difference between squat and stoop lifting technique using a number of kinematic variables. They had 12 females instrumented with EMG stand on a force plate and lift an $8.7 \mathrm{~kg}$ box from the floor to a waist height table using the squat and stoop lifting techniques at two different speeds (fast and slow). The results of their study demonstrated that there was no significant difference between the level of perceived exertion for lifting technique or speed (Kjellberg, Lindbeck, \& Hagberg, 1998). They also found no significant difference in peak moment at L5/S1 between the two lifting techniques. However, they did note that the peak moment at L5/S1 significantly increased for the fast lifts.

Although the stoop lifting technique is often deemed as the incorrect way to lift and a sure cause of LBD, it is interesting to note one study where reported pain was examined. In the study, 303 male workers were interviewed to determine various aspects of work history and environment that may lead to LBD (Damkot, Pope, Lord, \& Frymoyer, 1984). Surprisingly, twothirds of the individuals who did not report LBD used the stoop lifting technique. As a result of the conflicting findings with regards to the stoop and squat lifting technique, an alternate technique that combines aspects of both has been analyzed.

The semi-squat lifting technique has received some attention in the literature. Lifting a low lying object using the semi-squat lifting technique typically involves a moderate degree of trunk (approximately $45^{\circ}$ ) and knee flexion, with the feet kept flat on the ground (BurgessLimerick, Abernethy, \& Neal, 1995). The semi-squat lifting technique seems to be the preferred method in studies that have allowed participants to select their own lifting style (BurgessLimerick et al., 1995; Burgess-Limerick, 2003; Gagnon \& Smyth, 1992). This may be due to the 
fact that there is a reduced muscular effort while using the semi-squat technique (BurgessLimerick et al., 1995). In fact, a study by Burgess-Limerick and colleagues (1995) found that during the semi-squat lifting technique, there is an EMG silent period in the erector spinae. Individuals rated the semi-squat lifting technique as less tiring than the stoop lifting technique (Kumar, 1984) which, as mentioned, is less fatiguing than the squat lifting technique (Giat \& Pike, 1992; Straker, 2003). In terms of stress on the low back, Garg and Saxena (1985) deemed that the maximal accepted weight for the semi-squat lifting technique was $25 \%$ and $12 \%$ greater than the squat and stoop lifting techniques, respectively. The semi-squat lifting technique has produced such positive results that many health care professionals in Australia now report teaching the semi-squat technique rather than the squat technique (Straker, 2003). Based on the results of a number of studies, the semi-squat lifting technique may be an effective lifting technique in order to reduce the stress on the lumbar vertebral column while concurrently reducing muscular effort.

A variety of lifting techniques have been analyzed in the literature, each with positive and negative attributes. Although certain techniques are often described as being superior to another, and as a result taught in occupational settings, many researchers argue that individualized postures and patterns would be more beneficial than strict lifting guidelines (Albaladejo et al., 2010; Andrade, Araujo, \& Vilar, 2008; Burgess-Limerick, 2003; Cheng \& Chan, 2009) . Therefore, it is first necessary to understand how individuals lift naturally, without instruction, and whether there are differences between males and females. 


\subsection{Sex-Based Differences in Lifting Technique}

The majority of research with respect to lifting has been completed using solely male participants. Taking into account the anthropometric and strength differences between males and females, it is unlikely that the results from previous studies using only male participants can be generalized to females. Therefore, sex-based differences in lifting technique should be further examined.

Previous research has examined the difference between shear and compression force in males and females. Bejjani, Gross, and Pugh (1984) used a two-dimensional static model and found that back and knee shear forces were greater in females, and back compression at the $\mathrm{L}_{4} / \mathrm{L}_{5}$ joint was significantly greater for males, during a symmetrical lift. Stevenson, Greenhorn, Bryant, Deakin and Smith (1996) also examined differences in lifting technique between sexes using an incremental lifting machine (ILM) test and a maximal box lifting task. They found significant differences between males and females performing the ILM test in terms of timing, displacement, velocity, acceleration, force, and power. Marras, Davis, and Jorgensen (2002) examined differences in spine loading and musculoskeletal control strategies between males and females using an electromyography-assisted model. They found that when lifting a load, absolute spine compression was higher for males. However, under the restricted torso lifting condition, the majority of the differences were attributed solely to body mass discrepancies. Under the wholebody free dynamic condition, relative compression and anterior-posterior shear forces were

greater in females. The differences observed in the whole-body condition were attributed to muscle co-activation patterns and compensatory kinematic differences between males and females. 
Marras, Davis, and Jorgensen (2003), further investigated differences in lifting technique between sexes by having males and females exposed to similar workplace lifting demands. In their study, it was found that males had significantly greater compression forces than females. However, sex accounted for only $15 \%$, and $26 \%$ of the variability in compression and anteriorposterior shear, respectively. Task asymmetry and load weight had a greater impact on spinal loads than sex. Again, the sex differences in spine loading were attributed to kinematic trade-offs and muscle coactivity differences in combination with unequal body masses. In terms of kinematics, females used more hip motion, whereas much of the lifting motion for males originated from the lumbar spine.

Sex differences in lifting technique were examined directly by Lindbeck and Kjellberg (2001). In their study, participants were required to lift a handled box from the floor to a table located at the level of the umbilicus. Each participant performed two constrained lifting methods at two speeds. The load in the box was $12.8 \mathrm{~kg}$ for males, and $8.7 \mathrm{~kg}$ for females. The difference in load was assumed to correspond approximately to differences in physical capacity between males and females. Kinematic differences in lifting technique were found between sexes. More specifically, trunk angles were significantly greater for males for all lift conditions. However, a number of limitations were discussed by Lindbeck and Kjellberg (2001). Primarily, the biomechanical model that was used represented the trunk with one segment and, as a result, it may not have accurately represented the spinal curvature of the participants. A second limitation they outlined was that, although the load was different for males and females, it did not account for individual capabilities.

In 2011, Sadler, Graham, and Stevenson completed a study that addressed the limitations outlined by Lindbeck and Kjellberg (2001). Participants were instructed to lift continuously a 
handled box from the floor to a table positioned at $50 \%$ of the participant's height at a lifting rate of ten times a minute for three minutes using a freestyle (self-selected) lifting technique. They completed this twice; with a load of $0 \%$ and $10 \%$ of each individual's maximum isometric back strength. Using principal component analysis (PCA), no significant differences were found between males and females. The main limitation in the study was that participants were required to lift light loads, which may be why no differences in lifting technique were found. A second limitation is that participants were required to perform 30 continuous lifts, which may have led to a change in technique or fatigue.

\subsection{Summary}

Low back disorder has a very high prevalence in occupational settings (Dagenais et al., 2008; Hoy et al., 2010; LeBlanc \& LeBlanc, 2010). As a result, lifting technique has received much attention in biomechanics and ergonomics research. Earlier studies on lifting used solely male participants (Lindbeck \& Arborelius, 1991; Potvin et al., 1991; Wilke et al., 1999). However, due to the anatomical differences between males and females, and the rising prevalence of LBD in females, researchers began to differentiate lifting technique between sexes. The majority of studies have used discrete points on the lifting curve and set weights for each sex. From these studies, significant differences were found between males and females (Lindbeck \& Kjellberg, 2001; Stevenson et al., 1996). A recent study used principal component analysis (PCA) to analyze sex-based differences in lifting technique and found that males and females adopt similar strategies under light load conditions (Sadler et al., 2011). More research is needed to understand how males and females lift across increasing load conditions using more complex analysis techniques to analyze the curve shapes of lifting. Therefore, the objective of the current 
study is: 1) to determine if there is a sex-based difference in lifting technique across increasing load conditions; and, 2) to examine the use of body size-adjusted tasks and back strength-adjusted loads in the analysis of lifting technique. It is hypothesized that 1) males and females will use a similar lifting technique under light load conditions, and 2) differences in lifting technique between sexes will become apparent with increasing load. 


\section{Chapter 3}

\section{Methods}

\subsection{Participants}

Participants were recruited by use of posters on a university campus. Therefore, participants were university aged. Twenty-five participants (11 males and 14 females) participated in the study. Participants' descriptive statistics (age, height, and weight) are displayed in Table 1. All participants were healthy with no previous occupational experience in lifting or history of low back pain. There was no significant difference between groups for any of the descriptive statistics shown in Table 1 as determined by an independent samples t-test. Prior to testing, each participant read and signed an information and consent form which was approved by the Queen's University Research Ethics Board.

Table 1. Descriptive statistics for male and female participants

\begin{tabular}{lll|ll|c} 
& \multicolumn{2}{c|}{ Male } & \multicolumn{2}{c|}{ Female } & p value \\
\hline Age (years) & 24.13 & \pm 4.5 & 23.27 & \pm 1.9 & 0.579 \\
Weight $(\mathrm{kg})$ & 94.58 & \pm 43.6 & 75.03 & \pm 26.6 & 0.179 \\
Height $(\mathrm{cm})$ & 156.79 & \pm 40.4 & 163.54 & \pm 6.6 & 0.542 \\
\hline
\end{tabular}

\subsection{Experimental Protocol}

Each participant completed two testing sessions within one week of each other. During the first testing session the participants' maximum isometric back strength was obtained by 
having them complete a maximum back strength test using a modified functional capacity evaluation system (Arcon Vernova Inc., Saline, MI, USA) (Lotz et al., 2009; Sadler et al., 2011). Participants were secured to a horizontally oriented load cell and were instructed to maximally extend their back while keeping their lower body as relaxed as possible (Figure 1). Each participant completed 3 maximal exertions with 3 minutes of rest between trials. The maximum values for the three trials were averaged and used as a representation of the participants' reproducible maximum isometric back strength. Using the maximum isometric back strength, the lifting conditions were standardized to $10 \%, 20 \%$, and $30 \%$ of each participant's isometric back strength. Loads were standardized to control for the effects of the weight in the box and to examine whether results from previous research using solely male participants can be used to represent female participants. The three percentage loads were chosen based on a previous study by Sadler et al. (2011) where participants lifted a box loaded with $10 \%$ of their maximum back strength in order to prevent fatigue. The mean loads lifted for males and females are displayed in Table 2. There was a significant difference between males and females for all load conditions, as determined by an independent samples t-test. Following the back strength test, participants completed the experimental protocol to become familiar with the testing procedures. The lifting protocol was identical between the familiarization session and the testing session with the exception that the participants were not instrumented for data collection during the familiarization session. 
Table 2. Mean maximum isometric back strength and loads lifted by males and females

\begin{tabular}{lll|ll|c} 
& \multicolumn{2}{c|}{ Male } & \multicolumn{2}{c|}{ Female } & p value \\
\hline Maximum Back Strength (kg) & 72.00 & \pm 25.9 & 53.00 & \pm 10.4 & $\mathbf{0 . 0 2 0}$ \\
$10 \%$ Load $(\mathrm{kg})$ & 7.18 & \pm 2.7 & 5.21 & \pm 1.1 & $\mathbf{0 . 0 2 0}$ \\
$20 \%$ Load $(\mathrm{kg})$ & 14.36 & \pm 5.1 & 10.71 & \pm 2.1 & $\mathbf{0 . 0 2 4}$ \\
$30 \%$ Load $(\mathrm{kg})$ & 21.64 & \pm 7.8 & 15.86 & \pm 3.1 & $\mathbf{0 . 0 1 8}$ \\
\hline
\end{tabular}

For the second laboratory session, participants were instructed to wear running shoes, shorts, and a t-shirt. The lifting protocol required participants to perform a freestyle symmetric lift of a wooden-handled box $(36.7 \mathrm{~cm}$ x $29.2 \mathrm{~cm}$ x $25.5 \mathrm{~cm})$ with $3.0 \mathrm{~cm}$ diameter handles (Figure 2) loaded to $10 \%, 20 \%$, and $30 \%$ of their individual maximum isometric back strength. The order of load condition was randomized for each participant.

Each lift involved the participants lifting the box from a target on the floor to a target on a table (positioned at $50 \%$ of the participant's height) (Figure 3). Participants completed 5 separate lifts for each load condition $(10 \%, 20 \%$, and $30 \%$ of their individual maximum isometric back strength). One minute of rest was given between trials and a minimum of 3 minutes of rest was given between load conditions to prevent the effects of fatigue. Participants were free to choose their own lifting style with no step. Foot position was also self-selected and remained constant for all trials. Several practice lifts were required prior to data collection to ensure participants were familiar with the protocol and verify that the instrumentation was effective. 


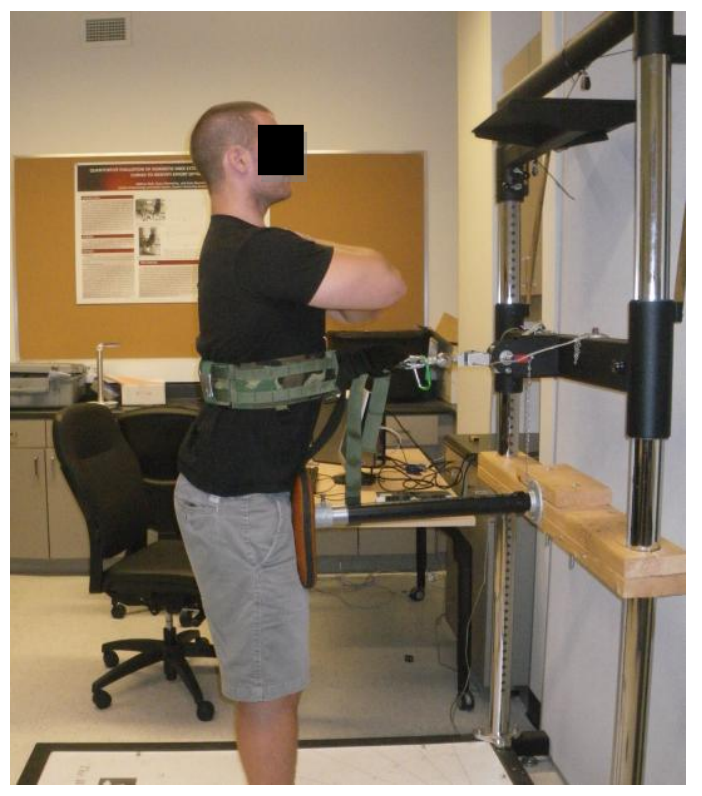

Figure 1. Participant completing a maximum back strength test against a horizontally-oriented load cell on the modified functional capacity evaluation system.

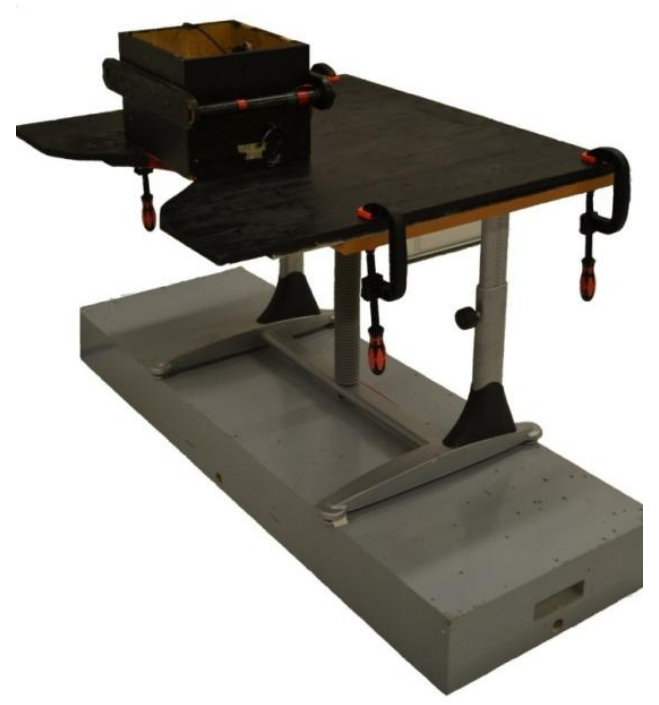

Figure 2. Experimental set up with a wooden handled box $(36.7 \mathrm{~cm}$ x $29.2 \mathrm{~cm}$ x $25.5 \mathrm{~cm})$ loaded to $10 \%, 20 \%$, and $30 \%$ of participant's maximum back strength, and table adjusted to $50 \%$ of participant's height 


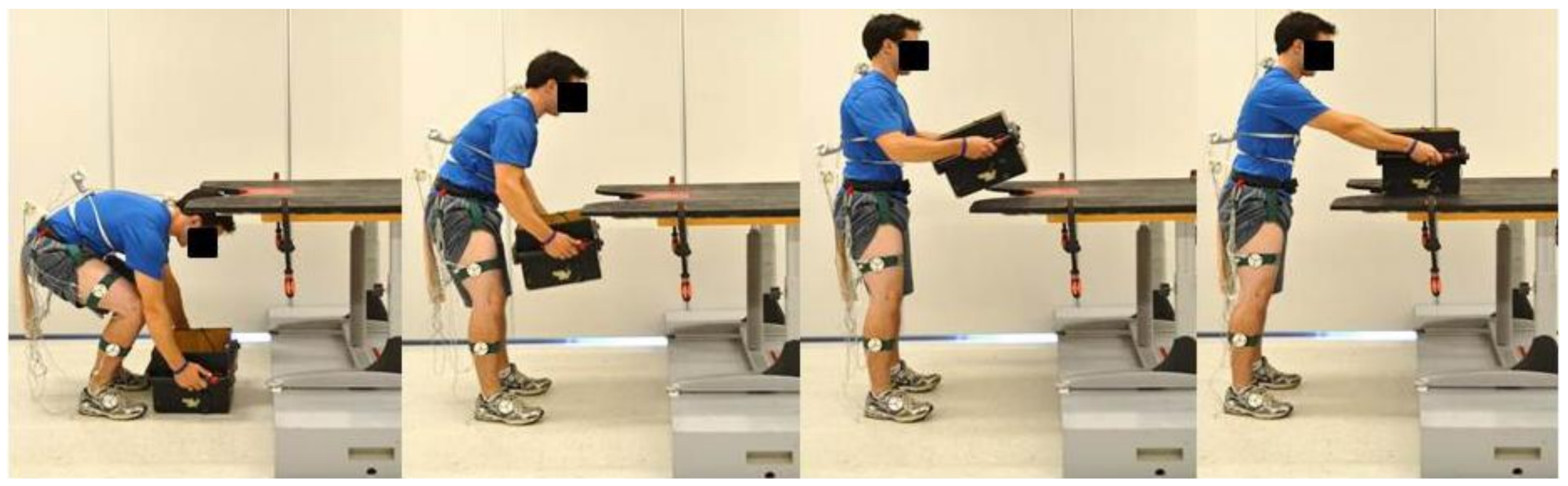

Figure 3. Participant completing one lift cycle. Participants were required to lift a handled box loaded with $10 \%$, 20\%, and 30\% of their maximum isometric back strength from a target on the floor to a target on a table (positioned at 50\% of their height) in a freestyle manner. 


\subsection{Instrumentation}

Instrumentation followed the same protocol as a study by Sadler et al. (2011). In order to decrease variability, the same researcher completed all instrumentation, and specific anatomical landmarks were used to determine marker placement. Participants were instrumented with six Infrared Emitting Diode (IRED) triads. Three of the triads were placed on the lateral side of the right foot, shank and thigh. The remaining 3 triads were placed on custom-made fins attached at the levels of $S_{1}, T_{12}$, and $C_{7}$ (Figure 4). Single IRED markers were placed on the right lateral malleolus and lateral femoral epicondyle (Figure 4). Prior to data collection, virtual markers were obtained using a probe of known dimensions at the $1^{\text {st }}$ and $5^{\text {th }}$ metatarsal of the right foot, right medial malleolus, right medial femoral epicondyle, symphysis pubis, right and left anterior superior iliac spine, and right posterior superior iliac spine. Virtual markers were necessary to calculate the ankle, knee, and hip joint centres using regression equations and were used to compute transformation matrices between marker triads and the corresponding anatomical coordinate systems (Allard, Cappozzo, Lundberg, \& Vaughan, 1997; Kirkwood, Culham, \& Costigan, 1999). The hip joint center was calculated based on a technique developed by Andriacchi and Strickland (1983) which was further improved upon by (Kirkwood et al., 1999). A reference posture was taken prior to data colletion to decrease variability and in order to calculate joint angles based on a neutral posture. 


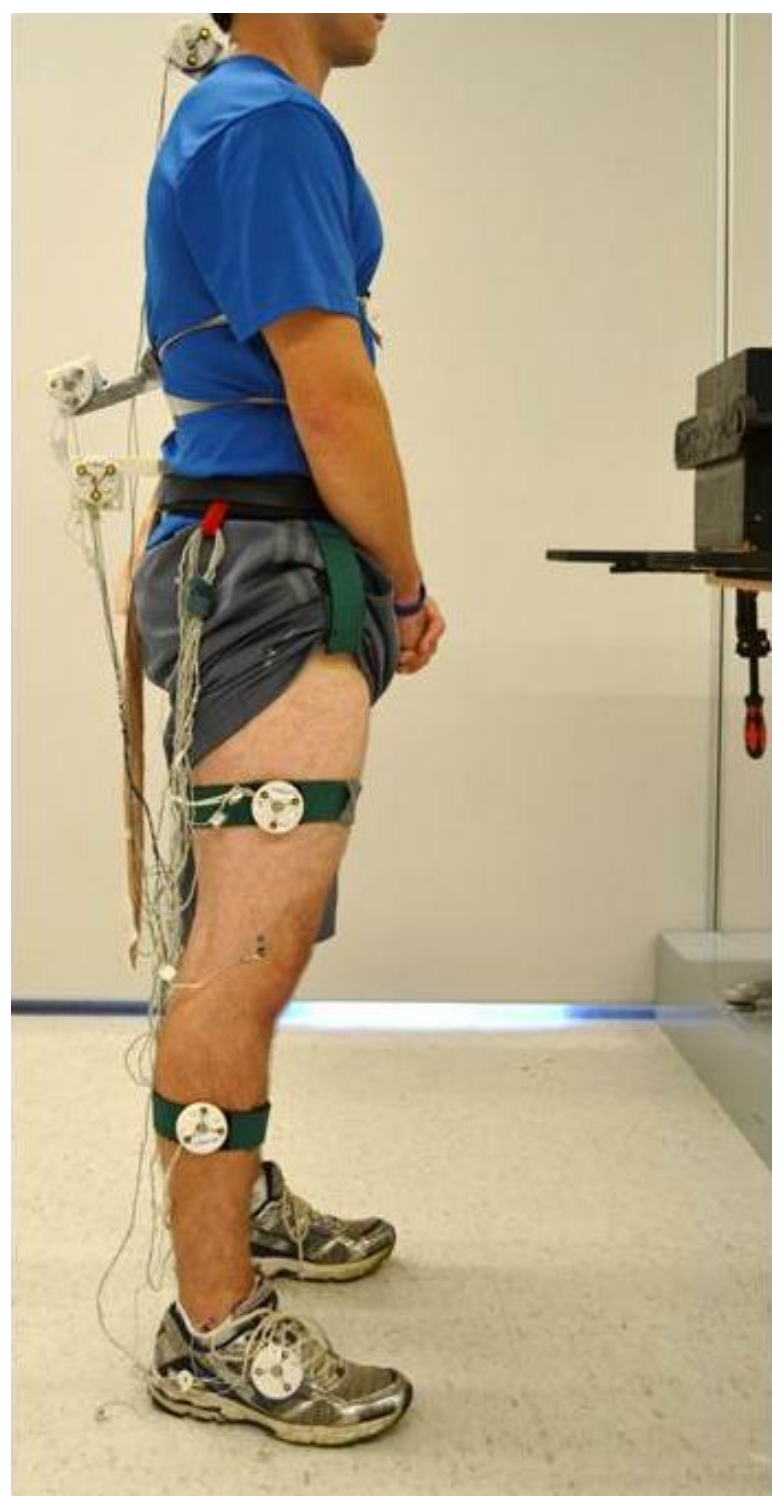

Figure 4. Participant instrumented with Infrared Emitting Diode (IRED) triads (lateral side of the right foot, shank and thigh, as well as on custom-made fins attached at the levels of S1, T12, and C7), and single IRED markers (lateral malleolus and epicondyle). 
A two camera Optotrak 3020 systems (NDI, Waterloo, ON) sampled at a frequency of $100 \mathrm{~Hz}$ was used to collect kinematic data. One camera was oriented perpendicular to the participant in the sagittal plane, while the other was located on an angle slightly behind the participant (Figure 5). The cameras were oriented in this fashion in order to have all IRED markers visible throughout the entire lift. Positioning and calibration of the Optotrak was completed prior to the arrival of the participant. The Optotrak data were used to calculate threedimensional joint angles of the ankle, knee, hip, lumbar spine, and thoracic spine using Euler rotation angles (Z-Y-X) (Winters \& Woo, 1990; Zatsiorsky, 1998; Zatsiorsky, 2002).

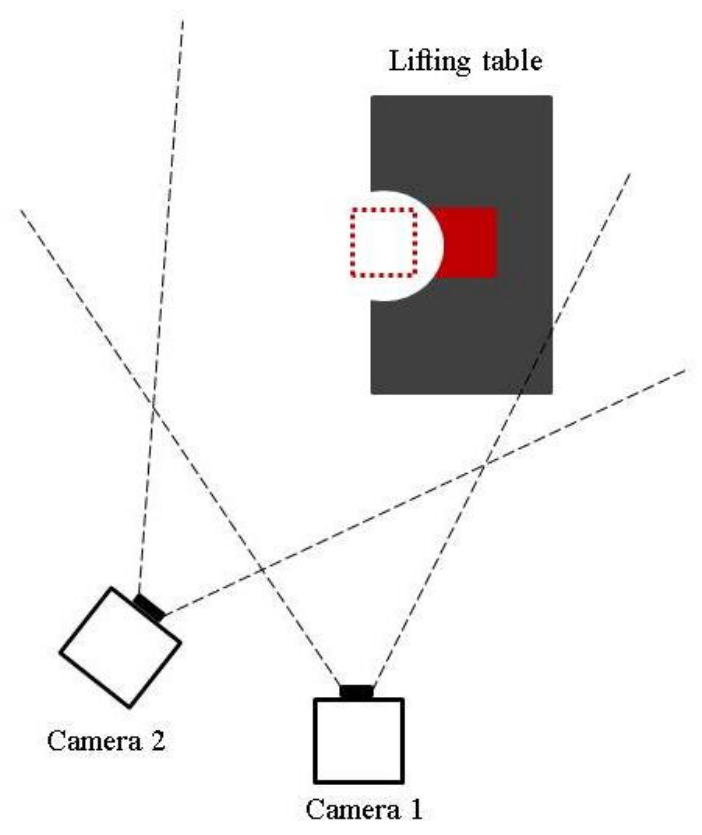

Figure 5. Experimental set up with one Optotrak 3020 (NDI, Waterloo, ON) camera oriented perpendicular to the sagittal plane lifting, and one camera oriented slightly behind and on an angle. The orientation of the cameras was chosen to have all makers visible throughout the entire lift. 
In order to capture the beginning (participant placing hands on the handle) and end of the lift (participant letting go of the box), the bottom of the box and the handles were instrumented with an analog switch (Figure 6). A collection time of 5 seconds was used to ensure the entire lift was captured. The collection for each trial was started with the participant in upright standing with their feet in their preferred starting position. The participant was then instructed to perform the lift. During the lift, one researcher watched the participant to ensure the lift was completed properly (i.e. beginning with their feet in the same position, avoiding hitting the table, etc.), while the other researcher observed the switch and marker data on a computer screen as a visual check of the instrumentation. After each lift, the data were checked by plotting the raw curves. The participant completed enough lifts to obtain 5 trials with proper switch and marker data.

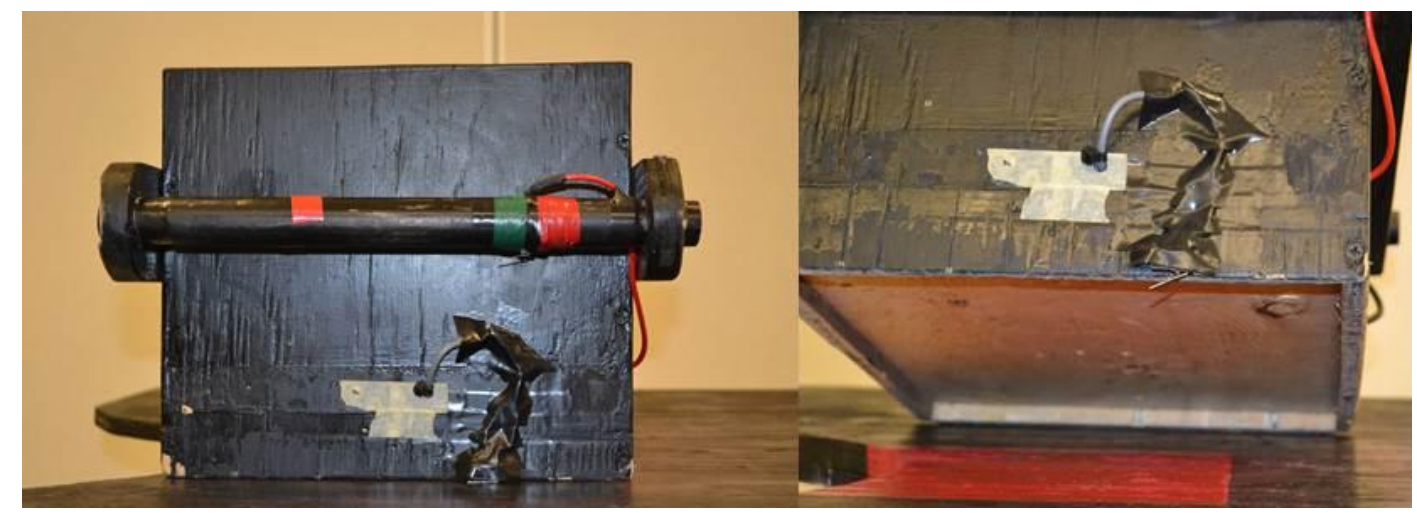

Figure 6. Box instrumented with two analog switches (handle and bottom of box) to indicate the start and end of the lift. 


\subsection{Data Processing}

All data processing calculation and analysis were completed using custom MatLab software (MathWorks, Natick, MA, USA). First, the data were filtered using a second order lowpass butterworth filter with a cutoff frequency of $10 \mathrm{~Hz}$. The cutoff frequency was determined to be optimal based on residual analysis. Using this cutoff frequency, noise was eliminated while retaining the important characteristics in the data. Following this, data were clipped from the point where the participant placed their hands on the handles to when they let go of the box. Therefore, the data consisted of the portion of the lift from the floor to the table. Joint angles of the ankle, knee, hip, lumbar spine, and thoracic spine were then calculated using three dimensional Euler rotations (Z-Y-X) (Zatsiorsky, 1998). The lower limb joint angles followed an anatomically-based coordinate system where the three principal axes were lateral-medial $(\mathrm{Z})$, posterior-anterior (Y), and distal-proximal (X) (Deluzio \& Astephen, 2007; Graham et al., 2011; Sadler et al., 2011). In order to calculate spine kinematics, the triad coordinate systems on the custom made fins $\left(\mathrm{S}_{1}, \mathrm{~T}_{12}\right.$, and $\left.\mathrm{C}_{7}\right)$ were used. The custom made fins were necessary in order to get an accurate three-dimensional representation of spinal movement during the symmetrical lift.

The directions of the coordinate systems are: positive $\mathrm{x}$-axis upward (twisting), positive $y$-axis forward (lateral flexion), and positive z-axis to the left (flexion-extension)(Zatsiorsky, 1998).

The angular positions were then normalized to $0-100 \%$ of the lift cycle. The five lifts were ensemble averaged to give a single representative waveform for each participant, for each load condition. The ensemble curves were placed in a matrix for analysis using PCA. Trials were eliminated if there was insufficient marker data to calculate joint angles or if the switch was not triggered properly. At minimum, 4 of the 5 trials were retained for each condition. 


\subsection{Statistical Method}

\subsubsection{Principal Component Analysis}

The main reason for using principal components analysis (PCA) is to summarize the most important information in the data (Deluzio, Wyss, Zee, Costigan, \& Serbie, 1997; Deluzio, Wyss, Costigan, Sorbie, \& Zee, 1999; Deluzio \& Astephen, 2007; Khalaf, Parnianpour, Sparto, \& Barin, 1999; Nguyen \& Reynolds, 2009; Sadler et al., 2011; Wrigley, Albert, Deluzio, \& Stevenson, 2005; Wrigley, Albert, Deluzio, \& Stevenson, 2006). PCA is a quantitative method for achieving both data reduction and separating useful parameters from redundant ones (Nguyen \& Reynolds, 2009). This is accomplished by representing the observations and the variables simultaneously using a limited number of optimal principal components or features (Deluzio \& Astephen, 2007). The features are optimal in that they explain a maximal amount of variance in the original data set (Deluzio \& Astephen, 2007).

The process involves creating matrices of waveforms, such that every participant's waveform of data were entered as a row vector $\boldsymbol{n} \boldsymbol{X} \boldsymbol{p}$ representing $n$-observations (waveform) and $p$-variables (normalized time points) (Wrigley et al., 2006). For the present analysis, the 11 males and 14 females' average waveforms corresponding to each of the three load conditions $(10 \%, 20 \%$, and $30 \%$ of individual maximum isometric back strength) were entered as row

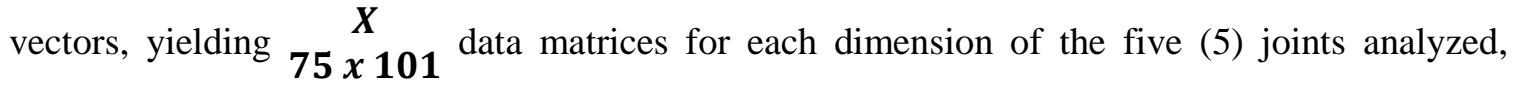
resulting in 15 principal component models displayed as: 


$$
\left[\begin{array}{cccc}
x_{1,1} & x_{1,2} & \ldots & x_{1,101} \\
x_{2,1} & x_{2,2} & \ldots & x_{2,101} \\
\vdots & \vdots & \ddots & \vdots \\
x_{75,1} & x_{75,2} & \cdots & x_{75,101}
\end{array}\right]
$$

The matrices were then analyzed using PCA. The detailed steps involved in PCA are reported in Appendix D. Interpretation of PCA is achieved through visual inspection of the PC loading vector, as well as waveforms of participants that scored low $\left(5^{\text {th }}\right.$ percentile $)$ and high $\left(95^{\text {th }}\right.$ percentile) on each PC (Astephen, Deluzio, Caldwell, Dunbar, \& Hubley-Kozey, 2008; Deluzio \& Astephen, 2007; Jones \& Rice, 1992; Reid, Graham, \& Costigan, 2010; Sadler et al., 2011).

\subsubsection{Principal Component Elimination}

As mentioned previously, the interpretation of PCs is achieved through visual inspection of waveforms of participants who scored high and low (Astephen et al., 2008; Deluzio \& Astephen, 2007; Jones \& Rice, 1992; Reid et al., 2010; Sadler et al., 2011). This method is both difficult and subjective. Since the amount of variance explained decreases with each subsequent $\mathrm{PC}$, the interpretation of PCs becomes increasingly difficult. This is due to the fact that the raw waveforms used for visual inspection contain features associated with the preceding PC (Sadler et al., 2011). In order to enhance visual inspection of PCs, principal component elimination (PCE) was used to enhance visual inspection of the $5^{\text {th }}$ and $95^{\text {th }}$ percentile waveforms. A detailed explanation of principal component elimination can be found in Appendix E. This process uses an equation to remove features associated with preceding PCs from the raw waveforms in order to enhance interpretability. 


\subsubsection{Hypothesis Testing}

Statistical analysis of PC scores was completed using SPSS 18.0 (SPSS Corporation, Chicago, IL, USA). From previous research it has been argued that retaining the PCs that explain $90 \%$ of the variance in the data set is sufficient to capture the primary modes of variation in the waveform (Deluzio \& Astephen, 2007). Therefore, PC scores that met the $90 \%$ trace criteria were analyzed for significance using a repeated measures analysis of variance ANOVA. A mixed design was used, with a between-subjects factor of sex (male, female) and a within-subject factor of load $(10 \%, 20 \%$, and $30 \%$ of individual maximal isometric back strength). The dependent variables were the PC scores and the independent variables were load condition and sex. A p value of $\mathrm{p}=0.005$ was used to represent significance in order to reduce the number of variables and based on previous research by Sadler et al., (2011). 


\section{Chapter 4}

\section{Results and Discussion}

Thirty-two PCs were retained from the five joints and three axes in accordance with the 90\% trace criterion (Appendix F). When tested with a repeated measures ANOVA, there was no significant effect of sex, or interaction effect of sex and load for any of the 32 PCs retained (Appendix F). There was, however, a significant effect of load on a number of PCs. The significant PCs are displayed in Table 3. PC1 was significant for ankle flexion, knee flexion, and knee abduction. For hip flexion, PC1, PC2, and PC3 demonstrated a significant difference between loads and each will be discussed in turn. 
Table 3. Means, standard deviations, and p-values for principal component scores retained in accordance with a $90 \%$ trace criterion that demonstrated significance. A $p$ value of $p=0.005$ was used to determine significance and are bolded in this table.

\begin{tabular}{|c|c|c|c|c|c|c|c|c|c|c|c|}
\hline \multirow[b]{2}{*}{ Joint } & \multirow[b]{2}{*}{ Angle } & \multirow[b]{2}{*}{ PC } & \multirow{2}{*}{$\begin{array}{c}\text { Variance } \\
\text { Explained } \\
(\%)\end{array}$} & \multicolumn{4}{|c|}{ Sex p Value } & \multicolumn{4}{|c|}{ Load p Value } \\
\hline & & & & $10 \mathrm{v} 20$ & $10 v 30$ & $20 v 30$ & All Loads & $10 v 20$ & $10 v 30$ & $20 v 30$ & All Loads \\
\hline Ankle & Flexion & 1 & 62.1 & 0.326 & 0.276 & 0.708 & 0.349 & 0.275 & 0.001 & 0.001 & 0.001 \\
\hline \multirow[t]{2}{*}{ Knee } & Flexion & 1 & 71.7 & 0.929 & 0.314 & 0.273 & 0.499 & 0.038 & 0.004 & 0.257 & 0.005 \\
\hline & Abduction & 1 & 67.4 & 0.029 & 0.236 & 0.275 & 0.078 & 0.059 & 0.001 & 0.048 & 0.001 \\
\hline \multirow[t]{3}{*}{ Hip } & Flexion & 1 & 68.3 & 0.140 & 0.046 & 0.568 & 0.132 & 0.063 & 0.003 & 0.238 & 0.012 \\
\hline & & 2 & 21.3 & 0.648 & 0.040 & 0.011 & 0.028 & 0.001 & 0.001 & 0.008 & 0.001 \\
\hline & & 3 & 7.6 & 0.761 & 0.477 & 0.729 & 0.794 & 0.027 & 0.001 & 0.177 & 0.002 \\
\hline
\end{tabular}




\subsection{Ankle Flexion}

Figure 7A displays the average ankle flexion ensemble lifting profiles for the $10 \%, 20 \%$, and $30 \%$ maximum load conditions. Participants began the lift with $22.0^{\circ} \pm 7.6^{\circ}, 21.1^{\circ} \pm 8.2^{\circ}$, and $20.5^{\circ} \pm 6.8^{\circ}$ of ankle dorsiflexion from neutral standing for the $10 \%, 20 \%$, and $30 \%$ load conditions, respectively. The mean start position, along with the mean maximum, minimum, and ranges for each load condition are presented in Table 4. From the start position, individuals exhibited ankle plantar flexion until midway through the lift, followed shortly by a small amount of dorsiflexion, and plantar flexion to the end of the lift where the box was placed on the table.

The PC1 loading vector for ankle flexion is displayed in Figure 7B. The loading vector began as a negative value and continued to explain more variance in the data until a quarter of the way through the lift, then gradually increased towards zero (less loading value). The most significant value for the PC1 loading vector for ankle flexion occurred at $26 \%$ of the lifting cycle (Figure 7B). Through visual inspection of Figure 7B, it is evident that the loading vector for PC1 of ankle flexion was most important for load differentiation from approximately $10 \%$ to $35 \%$ of the lifting cycle.

Returning to the mean waveforms for all load conditions (Figure 7A), it is evident that at $26 \%$ of the lifting cycle (most negative loading value of PC1), the curves for the $10 \%$ and $20 \%$ load conditions are similar, whereas the waveform corresponding to the mean of the $30 \%$ load condition is visually different. This agrees with the repeated measures ANOVA in that the 30\% load condition was significantly different than the $10 \%(\mathrm{p}<0.005)$ and $20 \%(\mathrm{p}<0.005)$ load conditions (Table 3).

Figure $7 \mathrm{C}$ shows the waveforms for the participants who scored high $\left(95^{\text {th }}\right.$ percentile) and low $\left(5^{\text {th }}\right.$ percentile $)$ for PC1 of ankle flexion. From Figure $7 \mathrm{C}$ it is evident that a magnitude 
difference accounted for $\mathrm{PC} 1$ of ankle flexion. In addition, this $\mathrm{PC}$ represents a slight time shift in ankle plantar flexion. Clearly, participants who scored low on this PC began with less ankle dorsiflexion and plantar flexed more quickly. Participants who scored high on this PC tended to begin with more ankle dorsiflexion and plantar flexed more slowly than those who scored low (Figure 7C).

The $30 \%$ load condition resulted in significantly lower PC scores $(-11.75 \pm 53.9)$ than both the $10 \%(9.01 \pm 42.6)(\mathrm{p}<0.005)$ and $20 \%(2.73 \pm 58.9)(\mathrm{p}<0.005)$ load conditions (Table 3$)$. Therefore, individuals used less ankle dorsiflexion at the beginning of the lift, plantar flexed more quickly, and ended with a greater amount of plantar flexion when lifting a heavy load, as compared to a light and medium load (Figure 7A). 

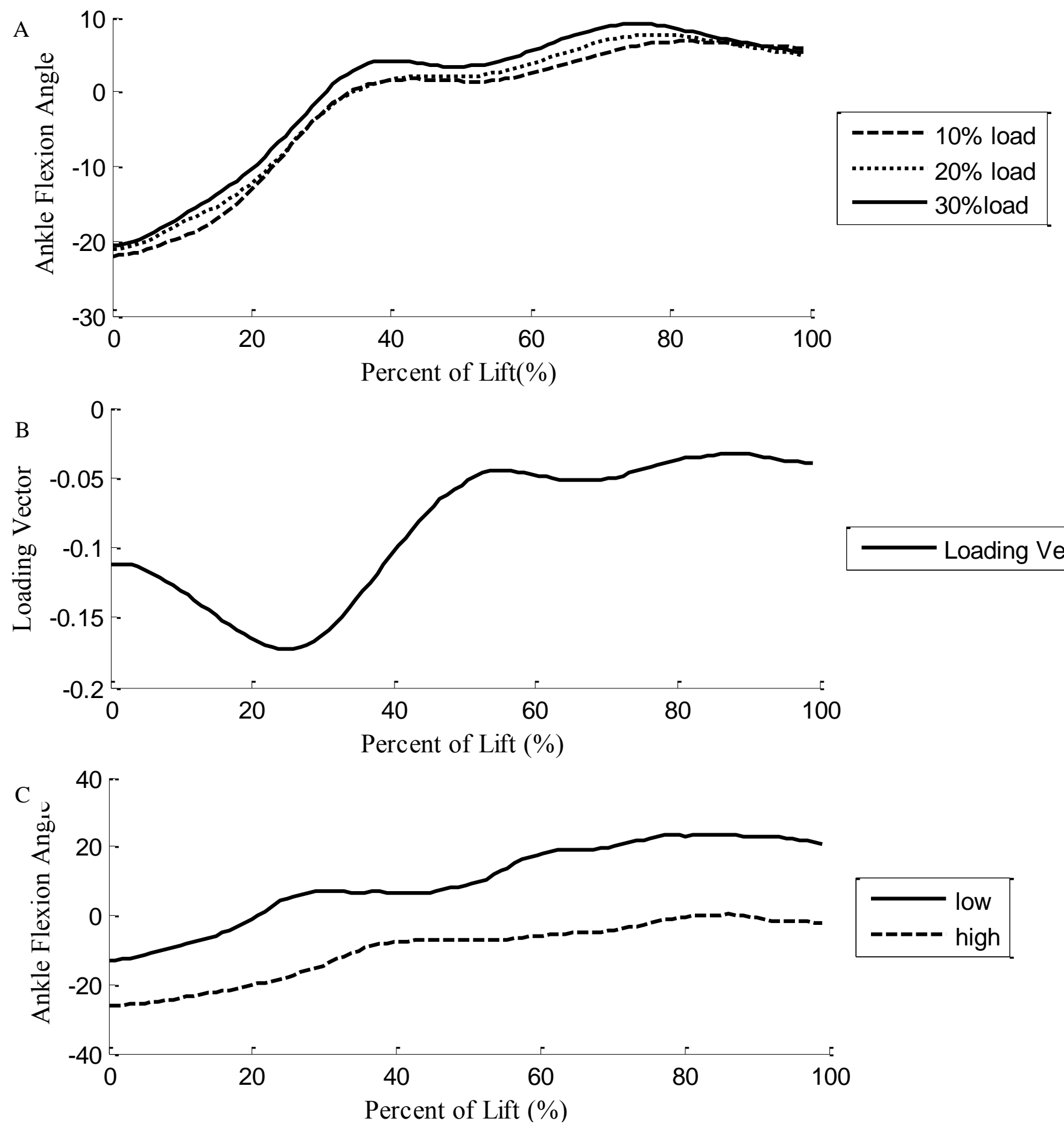

Figure 7. A) Mean ankle flexion angle during lift for 10\%, 20\%, and 30\% load conditions. Dorsiflexion is in the negative direction whereas plantar flexion is positive. B) Loading vector for ankle flexion principal component 1 (PC1). C) Ankle flexion waveforms for participants who scored high (95th percentile) and low (5th percentile) on PC1 of ankle flexion. Dorsiflexion is negative and plantar flexion is positive. 
Table 4. Mean maximum (degrees), minimum (degrees), range (degrees), and start position (degrees) for flexion/extension angles of the ankle, knee, hip, and lumbar, and thoracic spine. None of the discrete variables were significant across load conditions based on a repeated measures analysis of variance with a significance level of $\mathrm{p}<0.005)$.

\begin{tabular}{lccccccccc} 
Joint & Condition & \multicolumn{2}{c}{ Start } & \multicolumn{2}{c}{ Max } & \multicolumn{2}{c}{ Min } & \multicolumn{2}{c}{ Range } \\
\hline Ankle & & Mean & SD & Mean & SD & Mean & SD & Mean & SD \\
Flexion & $10 \%$ & -21.98 & 7.61 & 7.94 & 3.81 & -22.15 & 7.60 & 30.09 & 7.88 \\
& $20 \%$ & -21.12 & 8.25 & 9.45 & 5.11 & -21.30 & 8.21 & 30.75 & 8.08 \\
& $30 \%$ & -20.49 & 6.77 & 11.80 & 5.42 & -20.77 & 6.71 & 32.57 & 6.94 \\
\hline Knee & $10 \%$ & 93.56 & 23.30 & 94.46 & 23.30 & -3.15 & 6.90 & 97.61 & 25.02 \\
Flexion & $20 \%$ & 94.70 & 24.35 & 96.97 & 24.14 & -2.85 & 6.28 & 99.82 & 25.20 \\
& $30 \%$ & 98.08 & 21.64 & 101.49 & 20.82 & -2.84 & 6.32 & 104.33 & 22.16 \\
\hline Hip & $10 \%$ & -103.01 & 15.98 & -8.54 & 10.85 & -103.43 & 15.85 & 94.89 & 12.57 \\
Flexion & $20 \%$ & -103.78 & 14.96 & -7.13 & 13.11 & -104.68 & 14.88 & 97.55 & 13.00 \\
& $30 \%$ & -105.21 & 15.24 & -6.62 & 11.41 & -107.06 & 15.49 & 100.44 & 16.97 \\
\hline L Spine & $10 \%$ & -30.12 & 12.32 & -10.90 & 8.66 & -32.58 & 11.94 & 21.67 & 6.49 \\
Flexion & $20 \%$ & -28.85 & 12.62 & -11.77 & 9.48 & -31.90 & 12.04 & 20.13 & 6.98 \\
& $30 \%$ & -29.77 & 11.61 & -12.63 & 11.69 & -33.86 & 11.32 & 21.23 & 8.80 \\
\hline T Spine & $10 \%$ & 7.13 & 12.15 & 13.88 & 10.72 & -0.58 & 8.00 & 14.46 & 5.84 \\
Flexion & $20 \%$ & 9.27 & 13.66 & 16.09 & 13.08 & 0.38 & 9.22 & 15.70 & 6.83 \\
& $30 \%$ & 8.89 & 13.75 & 15.50 & 13.18 & -1.48 & 8.51 & 16.99 & 7.08 \\
\hline
\end{tabular}




\subsection{Knee Flexion}

There was no significant effect of sex for any of the 3 PCs retained for knee flexion. In terms of load, there was an overall significance for PC1 of knee flexion $(\mathrm{p}<0.005)$, as well as when comparing the $10 \%$ and $30 \%$ load conditions $(\mathrm{p}<0.005)$ (Table 3). The mean ensemble waveforms for each of the 3 load conditions are displayed in Figure 8A. When lifting a box from the floor to a table, individuals began the lift with a large knee flexion and rapidly extended their knees to place the box on the table. The mean starting knee flexion angle for the $10 \%, 20 \%$, and $30 \%$ load conditions were $93.6^{\circ} \pm 23.3^{\circ}, 94.5^{\circ} \pm 24.4^{\circ}$, and $98.1^{\circ} \pm 21.6^{\circ}$, respectively (Table 4 )

The loading vector for $\mathrm{PC} 1$ of knee flexion captured differences in range and rate of knee extension. The loading vector for this PC began with a negative value, and gradually became more meaningful up to its maximum loading vector at $22 \%$ of the lift (Figure $8 \mathrm{~B}$ ). From this point on, the loading vector increased rapidly towards zero (to approximately $60 \%$ of the lift) where it remained until the end of the lift (Figure 8B). As demonstrated by Figure 8C, individuals who scored high on this PC began with less knee flexion, extended their knee more rapidly, and ended with slightly more knee extension than those who scored low. Conversely, participants who scored low began with more knee flexion and ended with marginally less extension.

The mean PC score for PC1 of knee flexion for the 10\%, 20\%, and 30\% load condition was $25.67 \pm 116.1,-6.72 \pm 134.11$, and $-18.95 \pm 132.28$, respectively. The PC score for the $10 \%$ load condition was significantly higher than the PC score for the $30 \%$ load condition $(\mathrm{p}<0.005)$. Therefore, when lifting heavy loads, individuals begin with more knee flexion and have less knee extension velocity in comparison to lifting a light load (Figure 8C). 


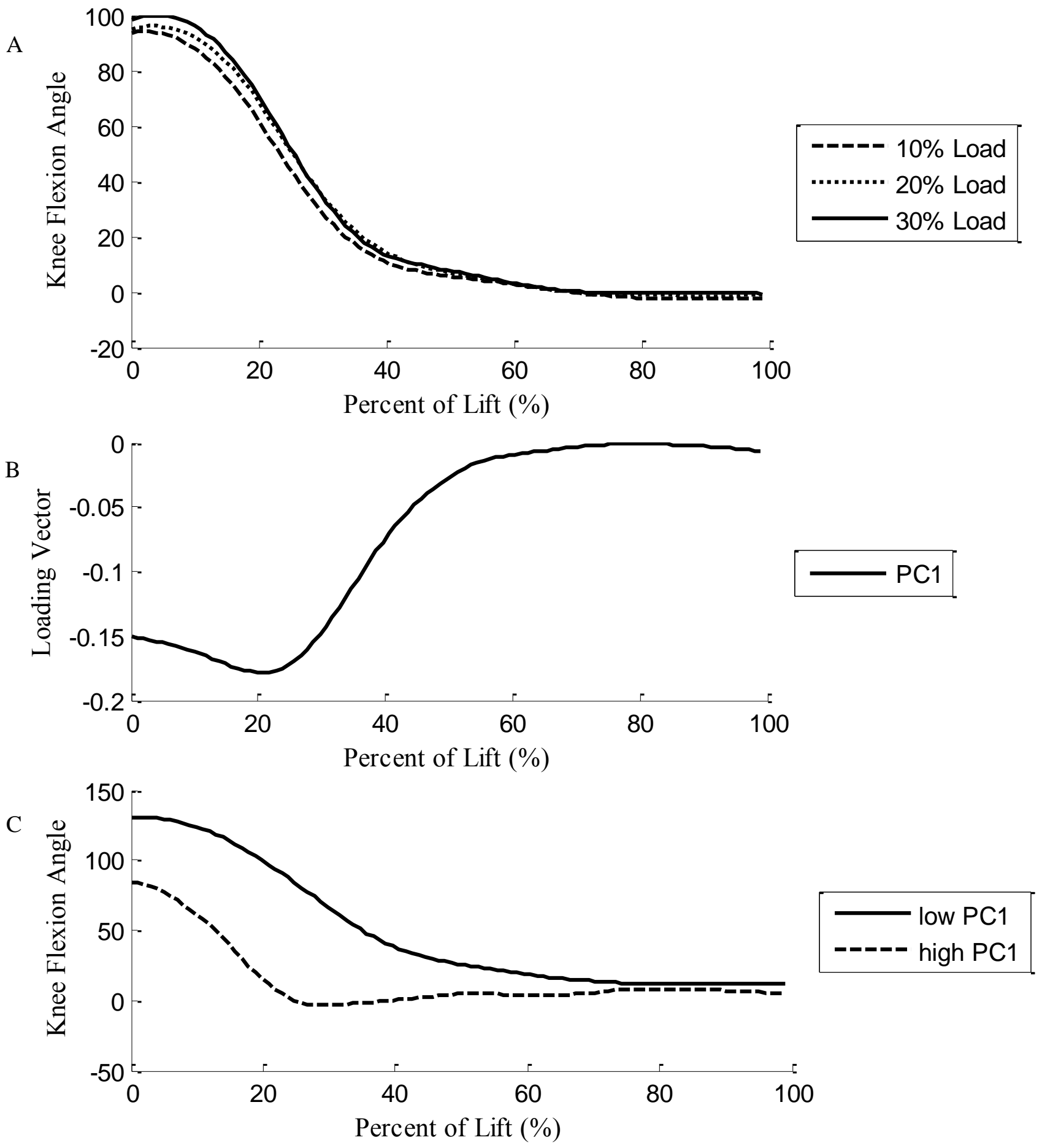

Figure 8. A) Mean knee flexion angle during lift for 10\%, 20\%, and 30\% load conditions. Knee flexion is positive and knee extension is negative. B) Loading vector for knee flexion principal component 1 (PC1). C) Knee flexion waveforms for participants who scored high (95th percentile) and low (5th percentile) on PC1. Knee flexion is positive, whereas extension is negative. 


\subsection{Knee Abduction}

Knee abduction is a term used in the literature to represent the knees being away from midline. Knee adduction therefore refers to the knees being towards midline. The mean ensemble waveforms for knee abduction for each of the 3 load conditions are displayed in Figure 9A. Similar to the previous angles discussed, participants began with a greater amount of knee abduction (knees out) for the $30 \%$ load condition $\left(12.3^{\circ} \pm 8.4^{\circ}\right)$ followed by the $20 \%\left(10.9^{\circ} \pm\right.$ $\left.10.0^{\circ}\right)$ and $10 \%\left(9.7^{\circ} \pm 7.9^{\circ}\right)$ load conditions (Table 4). From the start position, participants adducted their knees to neutral halfway through the lift cycle where they remained until the box was placed on the table.

The loading vector for PC1 of knee abduction is shown in Figure 9B. This loading vector followed a similar shape as the mean waveforms for the angle it described. It began with a large positive value, increased to explain the greatest amount of varianceat $15 \%$ of the lift and gradually had less weight following that point (Figure 9B). Examining the mean lifting profiles (Figure 9A) at $15 \%$ of the lift, it is evident that the $10 \%$ and $30 \%$ load conditions have a magnitude difference. However, there is also a difference in the timing of the lift. At the time of maximum loading vector (15\% of the lift), participants are well into knee adduction for the $10 \%$ load condition, where they are only beginning adduction for the $30 \%$ load condition. A graphical representation of participants who scored high and low for PC1 of knee abduction is shown in Figure 9C. Individuals who scored high began with their knees in abduction, and adducted their knees to neutral approximately midway through the lift (Figure 9C). Contrarily, those who scored low on this PC began with their knees in slight adduction and abducted their knees throughout the lift. 
Like the PC scores mentioned previously, there was a significant effect of load, but not sex for PC1 of knee abduction (Table 3). In terms of load, there was an overall effect of load $(\mathrm{p}<0.005)$, as well as a significant difference between the $10 \%$ and $30 \%$ load $(\mathrm{p}<0.005)$. The mean PC1 for the $10 \%$ load was $-9.44 \pm 41.7$, while the mean PC score for the $30 \%$ load was 9.22 \pm 43.8. Based on the results, it was evident that when individuals were lifting a heavy load they adopted more knee abduction at the beginning of the lift and adducted their knees to neutral midway through the lift. 


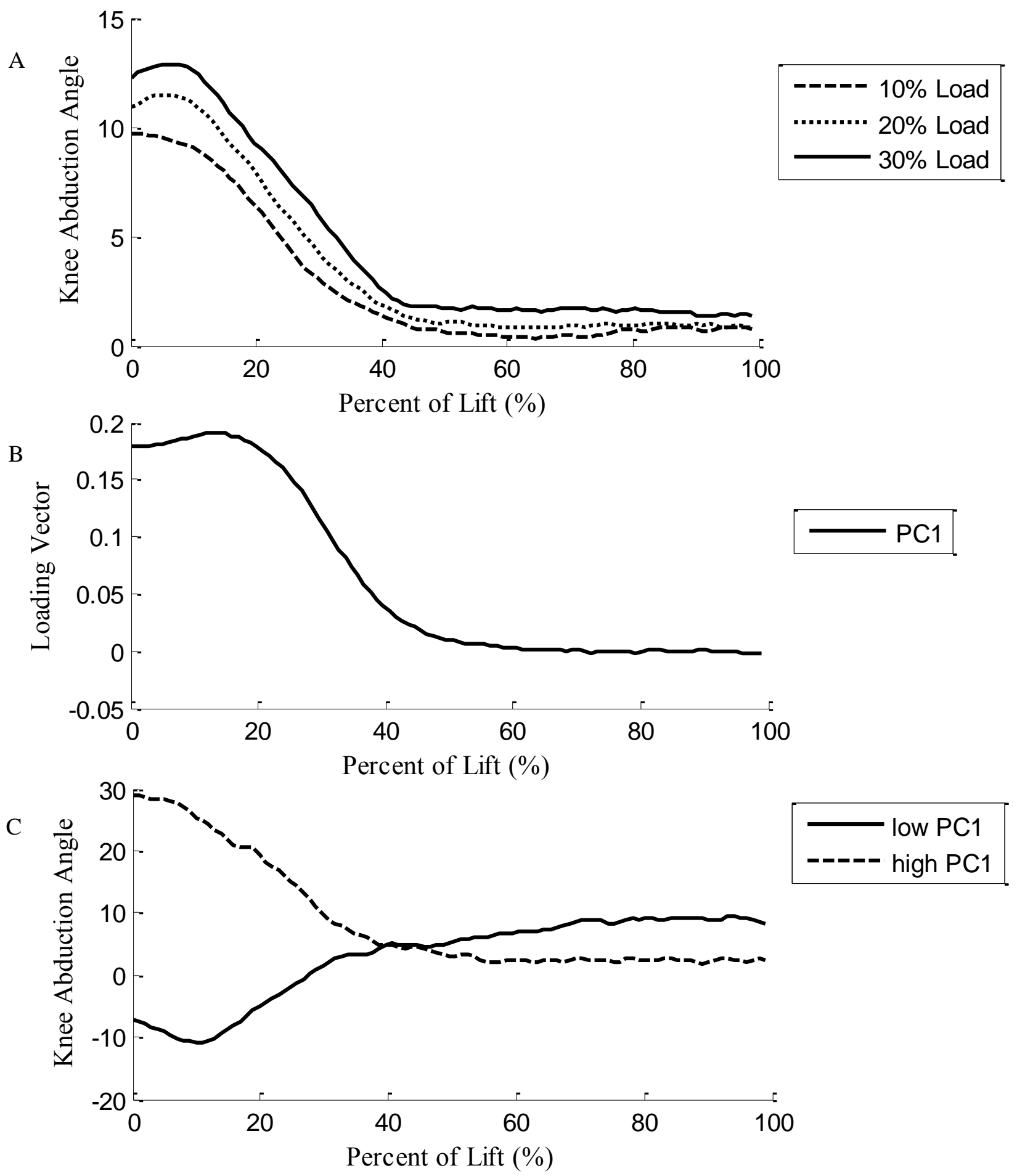

Figure 9. A) Mean knee abduction angle during lift for 10\%, 20\%, and 30\% load conditions. Knee abduction (knees away from midline) is positive, and knee adduction (knees towards midline) is negative B) Loading vector for knee abduction principal component 1 (PC1). C) Knee abduction waveforms for participants who scored high (95th percentile) and low (5th percentile) on PC1. Knee abduction is positive, whereas knee adduction is negative. 


\subsection{Hip Flexion}

Figure 10A demonstrates the mean lifting waveforms for the 10\%, 20\%, and $30 \%$ load conditions. Participants began the lift with a large hip flexion angle $\left(103.0^{\circ}, 103.8^{\circ}\right.$, and $105.2^{\circ}$ for the $10 \%, 20 \%$, and $30 \%$ load conditions, respectively) (Table 4), and rapidly extended their hips through to approximately $60 \%$ of the lift. From this point forward, participants flexed their hips slightly to place the box on the table, with the amount of flexion observed decreasing with increasing load condition (Figure 10A). Three PCs were required to explain 90\% of the variance in the hip flexion waveform, and all demonstrated a significant effect of load, but not sex. Each PC will be discussed in turn to explain the findings.

\subsubsection{Principal Component 1 (PC1)}

PC1 of hip flexion was considered a magnitude operator. The loading vector for PC1 is shown in Figure 10B. Visual analysis demonstrated that the loading vector was a negative value that remained quite consistent throughout the entire lift cycle, with the exception of a small decrease around $40 \%$ of the lift (Figure 10B). By comparing participants who scored high and low on PC1 (Figure 10C), it was evident that individuals who scored high on this PC completed the entire lift with less hip flexion than those who scored low.

For PC1 of hip flexion, a significant difference was only observed when comparing the $10 \%$ and $30 \%$ load conditions $(\mathrm{p}<0.005)$ (Table 3). The mean PC score for the $10 \%$ load conditions was $-19.31 \pm 118.8$, and the mean PC score for the $30 \%$ load conditions was $18.2 \pm$ 101.2. Since the PC scores for the $10 \%$ load condition was significantly lower than the $30 \%$ load condition, it was evident that individuals use less hip flexion when lifting a light load as 
compared to a heavy load. This finding was in agreement with the mean waveform data presented in Figure 10A.

\subsubsection{Principal Component 2 (PC2)}

PC2 of hip flexion demonstrated an overall effect on load $(\mathrm{p}<0.005)$ as well as a significant difference between the $10 \%$ load condition and both the $20 \%(\mathrm{p}<0.005)$ and $30 \%$ $(\mathrm{p}<0.005)$ load conditions. Figure 10D is a graphical representation of the PC2 loading vector. The loading vector began as a negative value and reached its maximal negative value at $27 \%$ of the lifting cycle. The loading vector then increased, intersected zero at $44 \%$ of the lift, and increased rapidly to $60 \%$ of the lift. From $60 \%$ to the end of the lift, the loading vector gradually increased (Figure 10D).

Figure 10E exhibits participants who scored high and low for PC2 of hip flexion. Examination of these curves demonstrates that this PC is a difference operator (range of motion). Individuals who scored high on PC2 began with a greater amount of hip flexion, and ended with a smaller amount of hip flexion than individuals who scored low (Figure 10E). This was especially evident in that the waveforms for participants who scored high and low cross at $44 \%$ of the lift, which corresponded to the zero point for the loading vector for this PC (Figure 10E).

The mean PC scores for the $10 \%, 20 \%$, and $30 \%$ load conditions were $-30.49 \pm 52.5,3.30$ \pm 58.0 , and $27.18 \pm 68.3$, respectively. Thus, individuals tended to use less hip flexion range when lifting a light load as compared to a medium and heavy load. 


\subsubsection{Principal Component 3 (PC3)}

Figure 10F illustrates the loading vector for PC3 of hip flexion. The loading vector began as a significant descriptor, decreased in power to $20 \%$ of the lift, and became a greater factor following this point to approximately $70 \%$ of the lift (Figure 10F). The minimum loading vector was seen at $0 \%$ of the lift and the maximum loading vector value occurred at $41 \%$ of the lift. The lifting waveforms for individuals who scored high and low on PC3 is depicted in Figure 10G. Individuals who scored high on this PC began with more hip flexion and extended their hips more rapidly than individuals who scored low on this PC.

In terms of load condition, there was only a significant difference between the $10 \%$ and $30 \%$ conditions $(\mathrm{p}<0.005)$ (Table 3$)$. The mean PC score for the $10 \%$ load was $11.56 \pm 30.7$, and the mean PC score for the $30 \%$ load was $-10.08 \pm 40.1$. Therefore, individuals tended to extend their hip with a greater velocity when lifting the $10 \%$ load, as compared to the $30 \%$ load. 

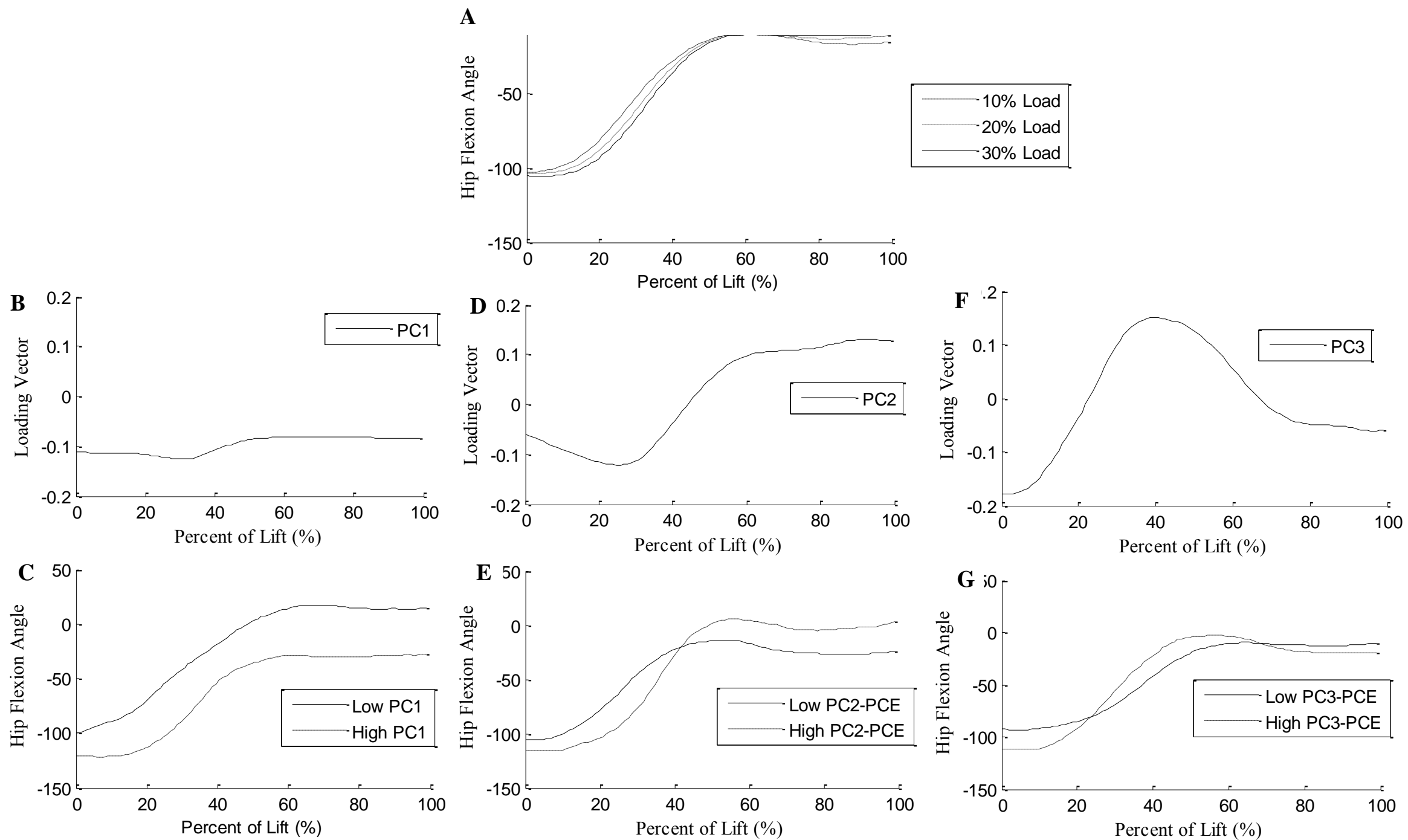

Figure 10. A) Mean hip flexion angle during the lift for 10\%, 20\%, and 30\% load conditions. Hip flexion is positive, and hip extension is negative B) Loading vector for hip flexion principal component 1 (PC1). C) Hip flexion waveforms for participants who scored high (95th percentile) and low (5th percentile) on PC1. Hip flexion is positive, whereas hip extension is negative D) Loading vector for hip flexion PC2. E) Hip flexion waveforms for participants who scored high and low on PC2 using principal component elimination (PCE). Hip flexion is positive and extension is negative F) Loading vector for hip flexion PC3. G) Hip flexion waveforms for participants who scored high and low on PC3 using PCE. Flexion is positive and extension is negative. 


\subsection{Lift Time}

There was no significant difference between males and females in terms of the time to complete the lift (Table 5). A $\mathrm{p}$ value of $\mathrm{p}=0.005$ was used to represent a significant difference between groups. There was, however, a significant effect of load on lift time. More specifically, as load increased, so did lift time (Table 6).

Table 5. Mean lift time (seconds) between males and females for 10\%, 20\%, and $30 \%$ load conditions. No significant differences in time were found between males and females based on an independent samples t-test $(\mathrm{p}<0.05)$

\begin{tabular}{ccc|cc}
\multicolumn{3}{c|}{ Male } & \multicolumn{2}{c}{ Female } \\
\hline Load Condition & Mean & SD & Mean & SD \\
\hline $10 \%$ & 2.45 & \pm 0.46 & 2.47 & \pm 0.49 \\
$20 \%$ & 2.66 & \pm 0.52 & 2.67 & \pm 0.47 \\
$30 \%$ & 2.75 & \pm 0.51 & 2.89 & \pm 0.48 \\
\hline
\end{tabular}

Table 6. Mean lift time (seconds) for all participants for 10\%, 20\%, and 30\% load conditions. There was a significant difference between load conditions as determined by an independent samples t-test $(\mathrm{p}<0.05)$.

\begin{tabular}{lcccc} 
Load Condition & $10 \%$ & $20 \%$ & $30 \%$ & p value \\
\hline Mean lift time & $2.46 \pm 0.47$ & $2.66 \pm 0.48$ & $2.83 \pm 0.49$ & $\boldsymbol{p}<\mathbf{0 . 0 0 5}$
\end{tabular}




\section{Chapter 5}

\section{General Discussion}

Differences in lifting technique between males and females during a freestyle symmetrical lift were analyzed using PCA of three-dimensional joint angles. Light (10\% maximum isometric back strength), medium (20\% maximum), and heavy (30\% maximum) load conditions were used to analyze differences with increasing load demands. Ankle, knee, hip, lumbar spine, and thoracic spine joint angles were assessed yielding 15 PC models (5 joints x 3 orientations). Using the $90 \%$ trace criteria, a total of 32 PCs were retained to explain the lifting patterns (See Appendix F). A p value of $\mathrm{p}=0.005$ was used to represent a significant difference between variables. This $\mathrm{p}$ value was chosen in order to reduce the number of variables while capturing important differences between lifting waveforms.

A repeated measures ANOVA revealed that there was no significant difference in lifting technique between males and females for any of the PCs retained. In other words, males and females exhibit similar lifting kinematics in the ankle, knee, hip, lumbar spine, and thoracic spine during a freestyle symmetric lift when weights were normalized to individual maximum isometric back strength. There was, however, a significant effect of load on lifting technique as well as lift time (which will be discussed later). For each joint angle, the minimum number of PCs retained to describe load differences varied from one to three. The PCs retained mainly explained magnitude, time shift, range, and rate of various aspects of the lifting profiles. A significant difference was observed for the PCs retained for the lower limb. No differences were observed for spinal kinematics.

The findings of the present study contradicted the study by Lindbeck and Kjellberg (2001). In their study, 12 females and 10 males were instructed to lift a handled box from the 
floor to a table using two set lifting patterns (squat and stoop) and rates (fast and slow). Lindbeck and Kjellberg (2001) concluded that males and females exhibit significantly different techniques during lifting tasks. The main differences that they observed were for trunk and knee angular range of motion.

There are a number of differences between the study by Lindbeck and Kjellberg (2001) and the current one. First, the study used discrete points on the lifting curve and assumed these points reflected the important characteristics of the technique for each individual. The parameters chosen do not analyze the entire lifting waveform, and may not accurately reflect sex-based differences in lifting technique. Second, the study used set loads for males (12.8kg) and females $(8.7 \mathrm{~kg})$. Therefore, the methodology did not take into account individual strength characteristics. Third, the lifting technique was very constrained for participants in terms of technique and timing, which led the researchers to state that the lifts appeared unnatural (Lindbeck \& Kjellberg, 2001). As a result, the researchers stated that the findings may not optimally describe lifting technique as workers are rarely confined to a certain speed and technique while lifting. Fourth, the biomechanical model used only one segment to describe the trunk. Therefore, any differences in trunk motion between men and women could not have revealed any sex differences in spinal curvature.

The limitations in the study by Lindbeck and Kjellberg (2001), were addressed in the present study and are reflected in the results. First, the present study used PCA in order to reduce the dimensionality of the data set and analyze the entire lifting waveform. Second, load was standardized to $10 \%, 20 \%$, and $30 \%$ of each individual's maximum isometric back strength. In this way, inherent differences between individuals could be taken into account. Third, participants were able to adopt a freestyle lift with no time constraints. Finally, custom made fins were placed at the levels of T12 and C7 in order to provide an accurate representation of spinal kinematics throughout the lift. 
Like the study by Lindbeck and Kjellberg (2001) the majority of previous studies on lifting technique have used discrete points on the lifting curve (i.e., ranges, peak values, and moments and torques) to represent the entire lifting waveform. Studies analyzing gait have used waveform-based analysis methods, such as principal component analysis (PCA) for quite some time (Chau, 2001; Deluzio et al., 1997; Deluzio et al., 1999; Deluzio \& Astephen, 2007; Ramsay \& Silverman, 1997). It was not until more recently, however, that lifting studies have adopted similar methods (Khalaf et al., 1999; Nguyen \& Reynolds, 2009; Sadler et al., 2011; Wrigley et al., 2005; Wrigley et al., 2006).

Wrigley et al. (2005) demonstrated that by reducing waveform trajectories into summary variables such as the maximum value, the sensitivity of subsequent hypothesis testing is critically compromised and clinically relevant group differences may be lost entirely. The difficulty in detecting differences from discrete variables was also described by Lindbeck and Kjellberg (2001) who stated that the variable selected for describing the technique may vary considerably within a particular protocol. It is often difficult to interpret the analysis of all parameters simultaneously in a way that is clinically relevant (Deluzio et al., 1997) and the parameters may not be able to be identified easily or repeatedly when abnormal waveform shapes are involved (Deluzio et al., 1997). Waveform-based analysis methods have the advantage of utilizing the entire movement waveform (gait, lifting, etc.) to detect subtle changes in the movement pattern not discernible when examining discrete points (Deluzio et al., 1999).

The findings of the present study agree with the work by Sadler and colleagues (2011) who examined sex-based differences in lifting technique under light load conditions using waveform based analysis. Sadler et al. (2011) used PCA to analyze lifting kinematics by having participants lift a box loaded with $0 \%$ and $10 \%$ of their individual maximum isometric back strength at a rate of 10 beats per minute for 3 minutes, resulting in 30 continuous lifts. The 
researchers concluded that under light load conditions, when load was standardized to individual strength characteristics, there was no difference in lifting technique between males and females.

Although the study by Sadler et al. (2011) used the curve shape analysis strategy of PCA to examine lifting kinematics, standardized the load to individual back strength, and adopted a freestyle lifting technique, there were limitations. First, in order to reduce the effects of fatigue, Sadler et al. (2011) used a relatively light load condition. As a result, the loads may have been too light to create any detectable differences in lifting technique between loads and sex. Second, participants were required to complete 30 continuous lifts in order to acquire an accurate representation of the lifting waveform. However, the large number of repetitions used in the methodology coupled with no rest time may have caused some fatigue and altered the results. A final limitation is that the protocol used by Sadler et al. (2011) constrained the time to complete the lift. Because of this, participants were not free to use their preferred speed of lifting, and may have caused them to change other aspects of their lifting technique.

The lifting protocol is an area of improvement from the study by Sadler et al. (2011) as well. Sadler et al. (2011) allowed a freestyle symmetric lift, but restricted participants to a single speed. In order to improve external validity, the present study allowed participants to use a freestyle lifting technique with no instructions on timing or rate of the lift. In this way, the lifting profiles are a more accurate representation of individual preference when it comes to lifting various loads. It is interesting to note that, although participants were free to choose their own lifting rate, there was no significant difference between males and females in the amount of time taken to complete the lift for any of the three load conditions. This finding demonstrates that when analyzing lifting technique, it may not be necessary to constrain the time of the lift.

When analyzing the specific effects of load on lifting technique, Sadler et al. (2011) found significant differences in lumbar spine flexion and hip rotation. In terms of lumbar spine flexion, they found that, while lifting a light load (10\% of maximum isometric back strength), 
individuals use a smaller range of lumbar flexion throughout the lift compared to a no load condition ( $0 \%$ of maximum isometric back strength). These findings were interpreted as participants adopting a more semi-squat lifting technique with light loads, and a stoop lifting technique with no load. The second significant difference between load conditions was peak differences in hip rotation (Sadler et al., 2011). When individuals lifted a loaded box, they had greater peak internal and external hip rotation compared to an unloaded box. The observation of greater hip rotation was interpreted as further evidence to support a semi-squat lifting technique while lifting light loads. The interpretation was based on the findings of McGill, Hughson, and Parks (2000) who stated that during bending tasks when spine flexion is minimized, more hip rotation is required.

The current findings agree with those from Sadler et al. (2011) in that participants adopt more of a squat lifting technique with increasing load. However, there is a discrepancy in the joint rotations that demonstrated a significant difference. Sadler et al. (2011) observed differences in lumbar spine flexion and hip rotation whereas the current study found differences between load conditions for PC1 of ankle flexion, PC1 of knee flexion, PC1 of knee abduction, as well as PC1, PC2, and PC3 of hip flexion. PC1 of ankle flexion was interpreted to represent a magnitude and time shift in the lifting waveform. There was a total effect of load as well as a significant difference between the $30 \%$ load and both the $10 \%$ and $20 \%$ load conditions. From this PC it was evident that, when lifting a heavy load, individuals began the lift with slightly less ankle dorsiflexion, plantar flexed more quickly, and ended with a greater degree of plantar flexion compared to a light a and medium load.

Differences in starting position were analyzed by Burgess-Limerick and Abernethy (1997). They found no significant difference in the amount of ankle dorsiflexion at the start of a lift for a light $(2.5 \mathrm{~kg})$ and heavy $(10.5 \mathrm{~kg})$ load condition. Burgess-Limerick and Abernethy (1997) also found no significant effect of load on plantar flexion velocity (Burgess-Limerick \& 
Abernethy, 1997). However, the study by Burgess-Limerick and Abernethy (1997) had participants lift set loads $(2.5 \mathrm{~kg}$ and $10.5 \mathrm{~kg})$, which were much less than the loads lifted in the present study. In the present study, the loads for the $10 \%$ condition averaged $7.18 \pm 2.7 \mathrm{~kg}$ for males and $5.32 \pm 1.1 \mathrm{~kg}$ for females. The mean loads for the $20 \%$ load condition were $14.35 \pm$ $5.1 \mathrm{~kg}$ for males and $10.71 \pm 2.1 \mathrm{~kg}$ for females. Finally, the average loads for the $30 \%$ load condition were $21.64 \pm 7.8 \mathrm{~kg}$ and $15.86 \pm 3.1 \mathrm{~kg}$ for males and females, respectively. Therefore, the amount of weight lifted by participants in the study by Burgess-Limerick and Abernethy (1997) might not have been different enough to produce similar results. In addition, individual strength characteristics were not taken into account. The greater amount of plantar flexion observed in the present study at the end of the heavy lift was likely an attempt to keep the box close to the participant's body. The participants might have been able to use more arm strength to place the light and medium load boxes on the table, but were unable to do so while lifting the heavy load.

PC1 of knee flexion captured differences in range and rate of the lifting waveform. There was a total effect of load on PC1, as well as a significant difference between the $30 \%$ and $10 \%$ maximum load conditions. Interpretation of PC1 of knee flexion revealed that, when lifting a heavy load, participants began with more knee flexion and had a smaller knee extension velocity compared to lifting a light load. This can be interpreted to mean that as load increased, individuals tended to adopt a more squat-like lifting technique. This finding agrees with the study by Burgess-Limerick and Abernethy (1997) in that knee flexion increased with load. The findings of the present study also demonstrated that as load increased, participants flexed their knees more slowly. This finding complements the findings of Garg and Saxena (1985) who found that the stoop lifting technique was faster than the squat lifting technique.

The results of knee abduction further support the findings that as participants lifted heavier loads they adopted more of a squat lifting technique. PC1 of knee abduction was 
interpreted to represent a magnitude and difference in timing of various aspects of the lift. There was an overall effect of load for PC1 of knee abduction as well as a significant difference between the $10 \%$ and $30 \%$ load conditions. While lifting the heavy load, participants began the lift with their knees in abduction and then adducted them to neutral approximately midway through the lift. For the light load, participants began with less abduction, or even slight adduction, and progressed to neutral halfway through the lifting cycle. This was likely due to the fact that, since participants were using more of a squat lifting technique for the heavy load conditions, it was necessary for them to abduct their knees in order to make room for the box. This is optimal when lifting using a squat lifting technique because the load can then be kept close to the body (Anderson \& Chaffin, 1986).

PC1 of hip flexion was interpreted as a magnitude operator. A significant difference was only observed when comparing the $10 \%$ and $30 \%$ load conditions. From this PC, participants used less hip flexion when lifting a light load, compared to a heavy load. PC2 of hip flexion was significantly different between the $10 \%$ and $20 \%$ load conditions, as well as the $10 \%$ and $30 \%$ load conditions. There was also an overall effect of load for this particular PC. PC2 of hip flexion was interpreted to represent a difference in range of motion. Participants who scored high on this PC began with a greater amount of hip flexion and ended with a smaller hip flexion angle. Participants who scored low on this PC began with less hip flexion and ended with more. Therefore, since the mean PC scores for the $10 \%$ load condition were significantly lower than both the $20 \%$ and $30 \%$ load condition, it was evident that participants tended to use less hip flexion range when lifting a light load, and more when lifting a heavy load. The magnitude and range differences observed agree with previous studies where researchers found that as load increased there was a significant increase in hip flexion (Burgess-Limerick \& Abernethy, 1997). The current study provided further support to demonstrate that participants used a squat lifting technique for heavier loads, and more of a stoop lifting technique for light loads. 
PC3 of hip flexion captured differences in the slope of the lifting waveform. There was an overall effect, and a difference between the $10 \%$ and $30 \%$ load conditions. It was evident that as load increased, the rate of hip extension decreased. The findings of the current study as well as previous studies demonstrated that individuals use a stoop lifting technique when lifting light loads and adopted a semi-squat or squat lifting technique as load increased (Burgess-Limerick \& Abernethy, 1997; Giat \& Pike, 1992; Sadler et al., 2011). Therefore, the effect of load on rate of hip extension also agreed with previous studies that found the squat lifting technique to be slower than the stoop lifting technique (Garg \& Saxena, 1985).

It is important to note the differences in starting position for each of the PCs mentioned. The starting position in the current study corresponds to the point when the participant places their hands on the handles. For each angle observed, the participant positioned themselves differently for various loads. As load increased participants decreased the amount of ankle dorsiflexion. Whereas, knee, hip and thoracic spine flexion increased with greater loads. Therefore, in agreement with previous research (Burgess-Limerick \& Abernethy, 1997; Sadler et al., 2011), participants tended to adopt more of a squat lifting technique with increasing load.

In the present study the amount of time to complete the lift was different between load conditions. The findings of the present study demonstrated that as load increased, so too did the time required to complete the lift. This result agreed with the Garg and Saxena (1985) study where they stated that the average duration of both flexion (time taken to bend from normal standing to grasp a box) and extension (time to return to normal standing) increased significantly as load increased from $2.5 \mathrm{~kg}$ to $10.5 \mathrm{~kg}$.

Although this study improved upon the methodology from past research, limitations were present. The main limitation is present in the sample size and characteristics. The small sample size that included eleven males and fourteen females, might have resulted in additional variables being significant if there were a greater number of participants. In addition, there was no 
significant difference between males and females in both height and weight. Therefore, the finding that males and females adopt a similar lifting technique may be due to the similarities between groups. However, there was a significant difference in maximum isometric back strength between males and females. This demonstrates the different strength characteristics between sexes and further supports the need for standardized loads when analyzing lifting technique. Second, in order to increase external validity and to get an accurate representation of natural lifting technique, participants were allowed to perform a freestyle symmetric lift with no constraints on lift time or rate. This may have increased the variability in the waveforms and caused no differences to be observed between sexes. However, since PCA was used, this should not have affected the results. Also, since there was no significant difference between males and females in terms of the amount of time taken to complete the lift, it is unlikely that the selfselected rate had a major impact on the results. 


\subsection{Conclusion}

The objective of this study was to determine if there is a sex-based difference in lifting technique across increasing load conditions using principal components analysis. The hypotheses were that 1) males and females would use a similar lifting technique under light load conditions, and 2) differences in lifting technique between sexes would become apparent with increasing load. The first hypothesis was proven since male and female participants used a similar lifting technique for the light load condition (10\% of maximum isometric back strength). The second hypothesis was observed in that, lifting technique of the ankles, knees, and hips changed as a result of load. However, there was no significant difference in lifting technique between sexes.

The current study demonstrates that when load was standardized to individual back strength characteristics, males and females adopted a similar lifting technique regardless of the load being lifted. In addition, this study supports the body of knowledge that suggested individuals adopt a more squat-like lifting technique with increasing load. Another major implication of the findings related to research methodology. First, when comparing sex-based differences in movement patterns, load should be standardized to individual back strength rather than by sex. Second, findings from studies examining one sex might be used as an accurate representation of lifting technique for the other sex once actual load factors are taken into account. Future research should focus on using the standardized load mass protocol on other situations that involve lifting technique, such as asymmetric lifting or lifting with and without a step. 


\section{References}

Adams, M. A., \& Hutton, W. C. (1981). The relevance of torsion to the mechanical derangement of the lumbar spine. Spine, 6(3), 241-248.

Albaladejo, C., Kovacs, F.M., Royuela, A., Pino, R., \& Zamora, J. (2010). The efficacy of short education program and a short physiotherapy program for treating low back pain in primary care. Spine, 35(5), 483-496.

Allard, P., Cappozzo, A., Lundberg, A., \& Vaughan, C. (Eds.). (1997). Three dimensional analysis of human locomotion Toronto: John Wiley \& Sons.

Anderson, J.A. (1986). Epidemiological aspects of back pain. Journal of the Society of Occupational Medicine, 36, 90-94.

Anderson, C. \& Chaffin, D. (1986). A biomechanical evaluation of five lifting techniques. Applied Ergonomics, 17(1), 2-8.

Andrade, S.C., \& de Araujo, A.G.R. (2008). Back school for patients with non-specific chronic low-back pain: benefits from the association of an exercise program with patient's education. Reumatologica Portuguesa, 33, 443-450.

Andriacchi, T.P., \& Strickland, A.B (1983). Gait analysis as a tool to assess joint kinetics. In: Proceedings of NATO. Advanced Study Institute for Biomechanics of Normal and Pathological Articulating Joints. Lisbon, Portugal, 83-103

Astephen, J. L., Deluzio, K. J., Caldwell, G., Dunbar, M. J., \& Hubley-Kozey, C. L. (2008). Gait and neuromuscular pattern changes are associated with differences in knee osteoarthritis severity levels. Journal of Biomechanics, 41, 868-876. 
Badley, E. M., Rasooly, I., \& Webster, G. K. (1994). Relative importance of musculoskeletal disorders as a cause of chronic health-problems, disability, and health-care utilization findings from the 1990 Ontario Health Survey. Journal of Rheumatology, 21(3), 505-514.

Bazrgari, B., Shirazi-Adl, A., \& Arjmand, N. (2007). Analysis of squat and stoop dynamic lifting: Muscle forces and internal spinal loads. European Spine Journal, 16, 687-699.

Beck, T. J., Ruff, C. B., \& Scott, W. W. (1992). Sex differences in geometry of the femoral neck with aging: A structural analysis of bone mineral data. Calcified Tissue International, 50(1), 24-29.

Bejjani, F., Gross, C., \& Pugh, J. (1984). Model for static lifting: Relationship of loads on the spine and the knee. Journal of Biomechanics, 17, 281-286.

Bernard, B. (1997). Musculoskeletal disorders and workplace factors: A critical review of epidemiologic evidence for work-related musculoskeletal disorders of the neck, upper extremity, and low back. Cincinnati, OH: National Institute for Occupational Health and Safety.

Boussenna, M., Corlett, E., \& Pheasant, S. (1982). The relations between discomfort and postural loading at the joints. Ergonomics, 25(4), 315-322.

Brinkmann, P., Biggemann, M., \& Hilweg, D. (1988). Fatigue fracture of human lumbar vertebrae. Clinical Biomechanics, 3, 21-23.

Brooks, P. M. (2006). The burden of musculoskeletal disease - a global perspective. Clinical Rheumatology, 25(6), 778-781. 
Brown, M. D., Holmes, D. C., \& Heiner, A. D. (2002). Intraoperative measurement of lumbar spine motion segment stiffness. Spine, 27(9), 954-958.

Burgess-Limerick. (2003). Squat, stoop, or something in between? International Journal of Industrial Ergonomics, 31(3), 143-148.

Burgess-Limerick, \& Abernethy, B. (1997). Toward a quantitative definition of manual lifting postures. Human Factors, 39(1), 141-148.

Burgess-Limerick, Abernethy, B., \& Neal, R. J. (1995). Self-selected manual lifting technique: Functional consequences of the interjoint coordination. Human Factors, 37(2), 395-411.

Burgess-Limerick, \& Abernethy, B. (1997). Qualitatively different modes of manual lifting. International Journal of Industrial Ergonomics, 19(5), 413-417.

Chau, T. (2001). A review of analytical techniques for gait data. part 1: Fuzzy, statistical and fractal methods. Gait and Posture, 13(1), 49-66.

Cheng, W. C., Yang, R. S., \& Huey-Jen, H. S. (2001). Effects of gender and age differences on the distribution of bone content in the third lumbar vertebra. Spine, 26(8), 964-968.

Cheng, A.S., \& Chan, E.P. (2009). The effect of individual job coaching and use of health threat in a job-specific occupational health education program on prevention of work-related musculoskeletal back injury. Journal of Occupational and Environmental Medicine, 51(12), $1431-1421$. 
Choi, B. C. K., Tennassee, L. M., \& Eijkemans, G. J. M. (2001). Developing regional workplace health and hazard surveillance in the Americas. Pan American Journal of Public Health, 10, $376-381$.

Coyte, P. C., Asche, C. V., Croxford, R., \& Chan, B. (1998). The economic cost of musculoskeletal disorders in Canada. Arthritis Care and Research, 11(5), 315-325.

Dagenais, S., Caro, J., \& Haldeman, S. (2008). A systematic review of low back pain cost of illness studies in the United States and internationally. The Spine Journal, 8, 8-20.

Damkot, D. K., Pope, M. H., Lord, J., \& Frymoyer, J. W. (1984). The relationship between work history, work environment, and low back pain in men. Spine, 9, 395-399.

Davis, K. G., \& Heaney, C. A. (2000). The relationship between psychosocial work characteristics and low back pain: Underlying methodological issues. Clinical Biomechanics, 15(6), 389-406.

de Looze, M. P., Dolan, P., Kingma, I., \& Baten, C. T. M. (1998). Does an asymmetric straddlelegged lifting movement reduce the low back load? Human Movement Science, 17, 243-259.

de Looze, M. P., Kingma, I., Thunnissen, W., Van Wijk, M. J., \& Toussaint, H. M. (1994). The evaluation of a practical biomechanical model estimating lumbar moments in occupational activities. Ergonomics, 37, 1495-1502.

Deluzio, K.J. \& Astephen, J. L. (2007). Biomechanical features of gait waveform data associated with knee osteoarthritis: An application of principal component analysis. Gait and Posture, 25, 86-93. 
Deluzio, K.J., Wyss, U. P., Costigan, P. A., Sorbie, C., \& Zee, B. (1999). Gait assessment in unicompartmental knee arthroplasty patients: Principal component modelling of gait waveforms and clinical status. Human Movement Science, 18(5), 701-711.

Deluzio, K.J., Wyss, U. P., Zee, B., Costigan, P. A., \& Serbie, C. (1997). Principal component models of knee kinematics and kinetics: Normal vs. pathological gait patterns. Human Movement Science, 16(2-3), 201-217.

Ebbesen, E. N., Thomsen, J. S., \& Beck-Nielsen, H. (1999). Age- and gender-related differences in vertebral bone mass, density, and strength. Journal of Bone Mineral Research, 14(8), 1394-1403.

Fahrni, W. H., \& Trueman, G. E. (1965). Comparative radiological study of the spines of primitive population with north americans and north europeans. Journal of Bone Joint Surgery, 47, 522-555.

Feuerstein, M., Marcus, S. C., \& Huang, G. D. (2004). National trends in non-operative care for nonspecific back pain. The Spine Journal, 4(1), 56-63.

Freburger, J. K., Holmes, G. M., Agans, R. P., Jackman, A. M., Darter, J. D., \& Wallace, A. S. (2009). The rising prevalence of chronic low back pain. Archives of Internal Medicine, $169(3), 251-258$.

Gagnon, M., \& Smyth, G. (1992). Biomechanical exploration on dynamic modes of lifting. Ergonomics, 35(3), 329-345.

Garg, A., \& Herrin, G. D. (1979). Stoop or squat, a biomechanical and metabolical evaluation. American Institute for Industrial Engineers, 11, 293-302. 
Garg, A., \& Saxena, U. (1985). Physiological stresses in warehouse operations with special reference to lifting technique and gender: A case study. American Industrial Hygiene Association Journal, 46, 53-59.

Genaidy, A., \& Karwowski, W. (1993). The effects of neutral posture deviations on perceived joint discomfort ratings in sitting and standing postures. Ergonomics, 36(7), 785-792.

Giat, Y., \& Pike, N. (1992). Mechanical and electromyographic comparison between the stoop and the squat lifting methods. Journal of Safety Research, 23, 95-105.

Graham, R. B., Sadler, E. M., \& Stevenson, J. M. (2011). Does the personal lift-assist device affect the local dynamic stability of the spine during lifting? Journal of Biomechanics, 44(3), 461-466.

Hagen, K. B., \& Harms-Ringdahl, K. (1994). Ratings of perceived thigh and back exertion in forest workers during repetitive lifting using squat and stoop techniques. Spine, 19, 25112517.

Henry, Y. M., \& Eastell, R. (2000). Ethnic and gender differences in bone mineral density and bone turnover in young adults: Effect of bone size. Osteoporosis International, 11(6), 512517.

Hoy, D., March, L., Brooks, P., Woolf, A., Blyth, F., \& Vos, T. (2010). Measuring the global burden of low back pain. Journal of Clinical Rheumatology, 24, 155-165.

Hsiang, S. M., Brogmus, G. E., \& Courtney, T. K. (1997). Low back pain (LBP) and lifting technique - A review. International Journal of Industrial Ergonomics, 19(1), 59-74. 
Hsiang, S. M., \& Mcgorry, R. W. (1997). Three different lifting strategies for controlling the motion patterns of the external load. Ergonomics, 40(9), 928-939.

Hukuda, S., \& Kojima, Y. (2002). Sex discrepancy in the canal/body ratio of the cervical spine implicating the prevalence of cervical myelopathy in men. Spine, 27(3), 250-253.

Jager, M., Luttman, A., \& Laurig, W. (1991). Lumbar load during one-handed bricklaying. International Journal of Industrial Ergonomics, 8, 261-277.

Jones, M. C., \& Rice, J. A. (1992). Displaying the important features of large collections of similar curves. The American Statistician, 46(2), pp. 140-145.

Katz, J. N. (2006). Lumbar disc disorders and low-back pain: Socioeconomic factors and consequences. Journal of Bone Joint Surgery, 88, 21-24.

Kessler, R. C., Davis, R. B., Foster, D. F., Van Rompay, M. I., Walters, E. E., Wilkey, S. A., et al. (2001). Long-term trends in the use of complementary and alternative medical therapies in the united states. Annals of Internal Medicine, 135(4), 262.

Keyserling, W., Sudarsan, S., Martin, B., \& Haig, A. 1. (2005). Effects of low back disability status on lower back discomfort during sustained and cyclical trunk flexion. Ergonomics, 48(3), 219-233.

Khalaf, K. A., Parnianpour, M., Sparto, P. J., \& Barin, K. (1999). Feature extraction and quantification of the variability of dynamic performance profiles due to the different sagittal lift characteristics. Rehabilitation Engineering, Institute of Electrical and Electronics Engineers (IEEE) Transactions on, 7(3), 278-288. 
Kirkwood, R. N., Culham, E. G., \& Costigan, P. (1999). Radiographic and non-invasive determination of the hip joint center location: Effect on hip joint moments. Clinical Biomechanics, 14(4), 227-235.

Kjellberg, K., Lindbeck, L., \& Hagberg, M. (1998). Method and performance: Two elements of work technique. Ergonomics, 41(6), 798-816.

Kumar, S. (1984). The physiological cost of three different methods of lifting in sagittal and lateral planes. Ergonomics, 27(4), 425-433.

Kumar, S. (1990). Cumulative load as a risk factor for low back pain. Spine, 15(12), 1311-1316.

Kumar, S. (1994). Lumbosacral compression in maximal lifting efforts in sagittal plane with varying mechanical disadvantage in isometric and isokinetic modes. Ergonomics, 37(12), 1975-1983.

Kumar, S. (2001). Theories of musculoskeletal injury causation. Ergonomics, 44(1), 17-47.

LeBlanc, K. E., \& LeBlanc, L. L. (2010). Musculoskeletal disorders. Primary Care: Clinics in Office Practice, 37(2), 389-406.

Lemasters, G. K., Atterbury, M. R., Booth-Jones, A. D., Bhattacharya, A., Ollila-Glenn, N., \& Forrester, C. (1998). Prevalence of work related musculoskeletal disorders in active union carpenters. Occupational and Environmental Medicine, 55, 421-427.

Lindbeck, L., \& Arborelius, U. P. (1991). Inertial effects from single body segments in dynamic analysis of lifting. Ergonomics, 34(4), 421-433. 
Lindbeck, L., \& Kjellberg, K. (2001). Gender differences in lifting technique. Ergonomics, 44(2), 202-214.

Linton, S. J. (2000). A review of psychological risk factors in back and neck pain. Spine, 25(9), $1148-1156$.

Lotz, C., Agnew, M., Godwin, A., \& Stevenson, J. (2009). The effect of an on-body personal lift assist device (PLAD) on fatigue during a repetitive lifting task. Journal of Electromyography and Kinesiology, 19, 331-340.

Marras, W. (2008). Working back: A systems view. Hoboken, N.J.: Wiley-Interscience.

Marras, W., Davis, K., \& Jorgensen, M. (2002). Spine loading as a function of gender. Spine, $127,2514-1520$.

Marras, W., Davis, K., \& Jorgensen, M. (2003). Gender influences on spine loads during complex lifting. The Spine Journal, 3, 93-99.

Marras (2008). The working back: a systems view. John Wiley and Sons. New Jersey

Martimo, K.P., Verbeek, J., Karppinen, J., Furlan, A.D., Takala, E.P., Kuijer, P.P., Jauhiainen, M., \& Viikari-Juntura, E. (2008). Effect of training and lifting equipment for preventing back pain in lifting and handling: Systematic review. British Medical Journal, 336, 429-431.

Martin, B. I., Deyo, R. A., \& Mirza, S. K. (2008). Expenditures and health status among adults with back and neck problems. Journal of the American Medical Association, 299(6), 656664. 
Masharawi, Y., Rothschild, B., Dar, G., Pleg, S., Robinson, D., \& Breen, E. (2004). Facet orientation in the thoracolumbar spine: Three-dimensional anatomic and biomechanical analysis. Spine, 29, 1755-1763.

McGill, S. M. (2002). Low back disorders: Evidence-based prevention and rehabilitation (2nd ed.). Champaign, IL: Human Kinetics.

McGill, S. M., \& Norman, R. W. (1986). Partitioning of the L4-L5 dynamic moment into disc, ligamentous, and muscular components during lifting. Spine, 11, 666-678.

McGill, S. M., Hughson, R. L., \& Parks, K. (2000). Changes in lumbar lordosis modify the role of the extensor muscles. Clinical Biomechanics, 15(10), 777-780.

Nelson, D. I., Concha-Barrientos, M., Driscoll, T., Steenland, K., Fingerhut, M., \& Punnett, L. (2005). The global burden of selected occupational diseases and injury risks: Methodology and summary. American Journal of Industrial Medicine, 48(6), 400-418.

Ng, J. K., Richardson, C. A., \& Kippers, V. (1998). Relationship between muscle fiber composition and functional capacity of back muscles in healthy subjects and patients with back pain. Journal of Orthopedic Sports Physical Therapy, 27(6), 389-402.

Nguyen, T. C., \& Reynolds, K. J. (2009). Principal component analysis of lifting kinematics and kinetics in pregnant subjects. International Conference on Biomedical Engineering (ICBME), 23, 1888-1891.

Nordin, M., \& Frankel, V. H. (2001). Basic biomechanics of the musculoskeletal system (3rd ed.). Philadelphia, PA: Lippincott Williams \& Williams. 
Norman, R. W., Wells, R., Neumann, P., Frank, P., Shannon, H., \& Kerr, M. (1998). A comparison of peak vs cumulative physical work exposure risk factors for the reporting of low back pain in the automotive industry. Clinical Biomechanics, 13, 561-573.

Norton, B. J., Sahrmann, S. A., \& Van Dillen, F. L. (2004). Differences in measurements of lumbar curvature related to gender and low back pain. Journal of Orthopedic Sports Physical Therapy, 34(9), 524-534.

Papageorgiou, A. C., Croft, P. R., Thomas, E., Ferry, S., Jayson IV, M., \& Silman, A. J. (1996). Influence of previous pain experience on the episode incidence of low back pain: Results from the South Manchester back pain study. Pain, 66(2-3), 181-185.

Potvin, J. R., McGill, S. M., \& Norman, R. W. (1991). Trunk muscle and lumbar ligament contributions to dynamic lifts with varying degrees of trunk flexion. Clinical Biomechanics, $16,1099-1107$.

Potvin, J. R., Norman, R. W., \& McGill, S. M. (1991). Reduction in anterior shear forces on the L disc by the lumbar musculature. Clinical Biomechanics, 6, 88-96.

Punnett, L., Fine, L., Keyserling, W., Herrin, G., \& Chaffin, D. (1991). Back disorders and nonneutral trunk postures of automobile assembly workers. Scandinavian Journal of Work and Environmental Health, 17, 337-346.

Punnett, L., Pruss-Ustun, A., Nelson, D., Fingerhut, M., Leigh, J., \& Tak, S. (2005). Estimating the global burden of low back pain attributable to combined occupational exposures. American Journal of Industrial Medicine, 48, 459-469. 
Punnett, L., \& Wegman, D. H. (2004). Work-related musculoskeletal disorders: The epidemiologic evidence and the debate. Journal of Electromyography and Kinesiology, 14(1), 13-23.

Ramsay, J. O., \& Silverman, B. W. (1997). Functional data analysis. New York: Springer.

Reid, S., Graham, R.B., \& Costigan, P. (2010). Differentiation of young and older adult stair climbing gait using principal component analysis. Gait and Posture, 31, 197-203.

Rezcallah, A. T., Xu, R., \& Ebraheim, N. A. (2001). Axial computed tomography of the pedicle in the lower cervical spine. American Journal of Orthopedics, 31(1), 59-61.

Ryan, S., \& Fried, L. (1997). The impact of kyphosis on daily functioning. Journal of the American Geriatrics Society, 45(12), 1479-1486.

Sadler, E.M. (2010). Principal component analyses of joint angle curves to examine lifting technique (Thesis). Queen's University, Kingston, Ontario, Canada.

Sadler, E. M., Graham, R. B., \& Stevenson, J. M. (2011). Gender difference and lifting technique under light load conditions: A principal component analysis. Theoretical Issues in Ergonomics Science, 1-16.

Siegmund, G. P., Sanderson, D. J., \& Myers, B. S. (2003). Awareness affects the response of human subjects exposed to a single whiplash-like perturbation. Spine, 28(7), 671-679.

Sizer, P. S., \& James, C. R. (2008). Considerations of sex differences in musculoskeletal anatomy. In J. J. Robert-McComb, R. L. Norman \& M. Zumwalt (Eds.), (pp. 25-55). Totowa, N.J.: Humana Press. 
Smith, F. W., \& Smith, P. A. (2002). Musculoskeletal differences between males and females. Sports Medicine and Arthroscopy Review, 10, 98-100.

Statistics Canada (2011). Labour force survey estimates (LFS), by sex, age group, annual. Canadian Socio-Economic Information Management System (CANSIM) database.

Stevenson, J., Greenhorn, D., Bryant, J., Deakin, J., \& Smith, J. T. (1996). Gender differences in performance of a selection test using the incremental lifting machine. Applied Ergonomics, $27,45-52$.

Stevenson, J., Bryant, T., Greenhorn, D., Smith, T., Deakin, J., \& Surgenor, B. (1990). The effect of lifting protocol on comparisons with isoinertial lifting performance. Ergonomics, 33(12), $1455-1469$.

Straker, L. (2003). Evidence to support using squat, semi-squat and stoop techniques to lift low lying objects. International Ergonomics, 31, 149-160.

Toussaint, H. M., van Barr, C. E., van Langen, P. P., de Looze, M. P., \& van Dieen, J. H. (1992). Coordination of the leg muscles in the backlift and leglift. Journal of Biomechanics, 25, $1279-1289$.

Trimble, M. H., Bishop, M. D., \& Buckley, B. D. (2002). The relationship between clinical measurements of lower extremity posture and tibial translation. Clinical Biomechanics, 17(4), 286-290.

Truumees, E., Demetropoulos, C. K., \& Yang, K. H. (2003). Failure of the human cervical endplates: A cadaveric experimental model. Spine, 28(19), 2204-2208. 
van Dieen, J. H., Creemers, M., Draisma, I., Toussaint, H. M., \& Kingma, I. (1994). Repetitive lifting and spinal shrinkage, effects of age and lifting technique. Clinical Biomechanics, 9, $367-374$.

van Dieen, J. H., Hoozemans, M. J. M., \& Toussaint, H. M. (1999). Stoop and squat: A review of biomechanical studies on lifting technique. Clinical Biomechanics, 14, 685-696.

van Tulder, M., Koes, B., \& Bombardier, C. (2002). Low back pain. Baillière's Best Practice and Research in Clinical Rheumatology, 16(5), 761-775.

Walker, B. (2000). The prevalence of low back pain: A systematic review of the literature from 1966 to 1998. Journal of Spinal Disorders, 13(3), 205-217.

Wang, S. C., Brede, C., \& Lange, D. (2004). Gender differences in hip anatomy: Possible implications for injury tolerance in frontal collisions. Annual Proceedings from the Association for the Advancement of Automotive Medicine, 48, 287-301.

Welbergen, E., Kemper, H. C. G., Knibbe, J. J., Toussaint, H. M., \& Clijssen, L. (1991). Efficiency and effectiveness of stoop and squat lifting at different speeds. Ergonomics, 34, 613-624.

White, A., \& Panjabi, M. (1990). Clinical biomechanics of the spine (2nd ed.). Philadelphia, NY: J.B. Lippincott Company.

Wilke, H. J., Neef, P., Caimi, M., Hoogland, T., \& Claes, L. E. (1999). New in vivo measurements of pressures in the intervertebral disc in daily life. Spine, 24(8), 755-762. 
Winters, J. M., \& Woo, S. L. (1990). Multiple muscle systems - biomechanics and movement organization. Verlag, NY: Springer.

Woolf, A. D., \& Pfleger, B. (2003). Burden of major musculoskeletal conditions. Bulletin of the World Health Organization, 81, 646-656.

Wrigley, A., Albert, W., Deluzio, K., \& Stevenson, J. (2005). Differentiating lifting technique between those who develop low back pain and those who do not. Clinical Biomechanics, 20, 254-263.

Wrigley, A., Albert, W., Deluzio, K., \& Stevenson, J. (2006). Principal component analysis of lifting waveforms. Clinical Biomechanics, 21, 567-578.

Xu, R., Burgar, A., Ebraheim, N. A., \& Yeasting, R. (1999). The quantitative anatomy of the laminas of the spine. Spine, 24(2), 107-113.

Xu, Y., Bach, E., \& Ørhede, E. (1997). Work environment and low back pain: The influence of occupational activities. Occupational and Environmental Medicine, 54(10), pp. 741-745.

Yoganandan, N., \& Knowles, S. A. 1. M., D.J. (2003). Anatomic study of the morphology of human cervical facet joint. Spine, 28(20), 2317-2323.

Zatsiorsky, V. M., 1932-. (1998). Kinematics of human motion. Champaign, IL: Human Kinetics.

Zatsiorsky. V. (Ed.). (2002). Kinetics of human motion. Champaign, IL: Human Kinetics. 
Appendix A

Ethics Approval 
May 25, 2011

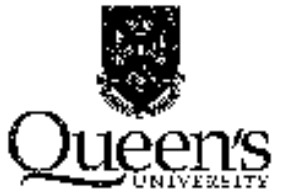

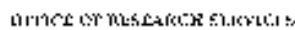

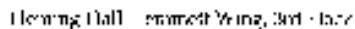

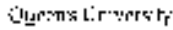

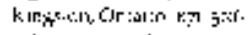

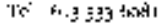

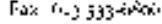

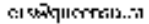

v':

Dr. Juarı Flever

School of Riztsiology ani Fres:llh Slıtlies

$2 \mathrm{~K}$ Dixision Sitreet

Quein's Universily'

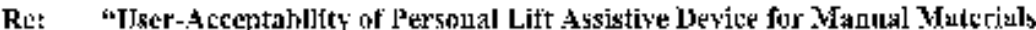

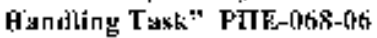

Dear' Dr. Ślcerctson,

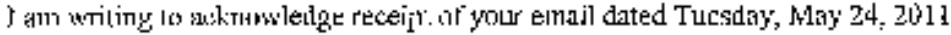
which includs the following:

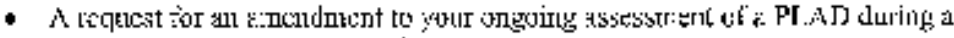
manus] mecer'als lianding tnsk

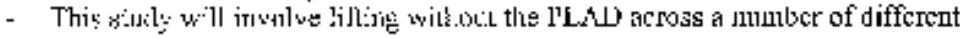
conditjons

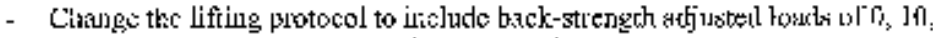
$20,30,40$ a $150 \%$ maxism back strcosh

- Coange llo lifirig problıtol hr irclude symmetric and asyametric lifs

- Aililitistr all Plillip shemprald to the sandy

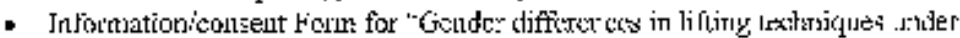

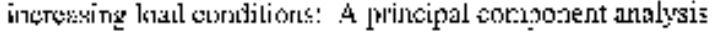

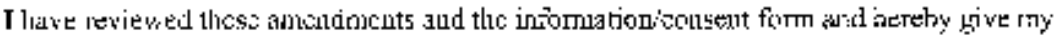

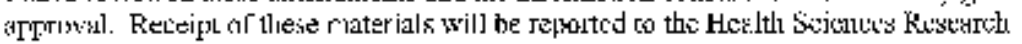
Etlois hoand.

Yolrs silucersiy,

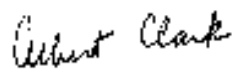

Alber: Clark, i'l.D.

Chair

Kustarish Ei.iis Buata

AFCikr

thirtk Research

think Queen's 
Appendix B

Ethics Letter of Information and Consent 


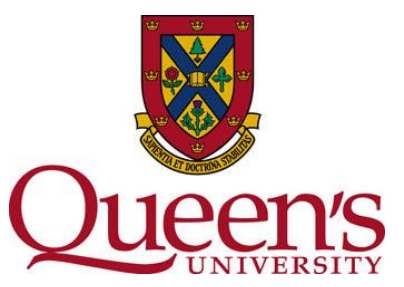

SCHOOL OF KINESIOLOGY AND HEALTH STUDIES (SKHS)

Physical Education Centre

Ethics Consent for

Kingston, Ontario, Canada K7L 3N6

\section{Research Study entitled:}

\section{Gender differences in lifting technique under increasing load conditions: A principal component analysis}

Dear Participant,

You are invited to participate in a research study to examine the differences in lifting technique between males and females. This Ethics Consent letter will present key information so you can read about the study and decide whether you wish to become a participant. If you wish to participate, you will be asked to sign this letter and leave a copy of the last page with the researcher.

\section{Aims and Purposes of the Study:}

The goals of this research are: a) to determine if males and females exhibit different lifting technique while lifting various loads, and b) if so, are these differences more pronounced when lifting heavier loads.

\section{Methodology:}

Personal Information: For your personal safety, we wish to exclude you from the study if you are currently suffering from shoulder, knee or low back pain.

Getting Ready for Testing: Prior to testing, you will be asked to complete the Physical Activity Readiness Questionnaire (PAR-Q). The PAR-Q helps determine if there is any health risk associated with your participation. In addition, we will need to measure your body weight and height to determine the load you will lift and how high to position the table. The testing task will require dynamic lifting; therefore, we would like you to complete a series of stretching exercises for the back, shoulder girdle and legs. This warm-up will require approximately 5 minutes.

Instrumentation: We will use a two-camera system called the Optotrak 3020 that captures 3dimensional coordinates of body landmarks in space. In order to track the body landmarks it is necessary to attach markers to the body part of interest. Some markers will be attached with Velcro or straps, while others will be digitized points from your body called virtual markers. The body landmarks we will be using are the foot, lower leg, upper leg, sacrum (S1), lower lumbar spine (L1) and thoracic spine (T1). Once the markers are in place, we will ask you to lift a 
wooden box with handles from floor to desktop height while standing on a force platform. We also have switch on the box to indicate the timing of the lift cycle.

Testing Protocol: In a single testing session, you will complete the same protocol 8 times: once for each of six load conditions, and once for each of the four lifting conditions. The five load conditions are: a) $0 \%$ body weight, b) $10 \%$ body weight, c) $20 \%$ body weight, d) $30 \%$ body weight, e) $40 \%$ body weight, and f) $50 \%$ body weight. The lifting conditions are a) symmetric nostep, b) symmetric with step, c) asymmetric no-step, and d) asymmetric with step. The order of the load conditions as well as the lifting styles will be randomized. All eight tasks use the same protocol. You will lift the handled-box from floor to a target on a shelf at 50\% your body height (approximately the belly button) either placed directly in front of you (symmetric) or slightly to the left of your body (asymmetric). Then you will return the box to the floor. This protocol will be repeated 5 separate times for each condition. For the no-step conditions, you will be asked to maintain a stationary position with your feet, and lift as naturally as possible. For the step conditions, you will be asked to take a step in order to lift the box from the floor and place it on the table. Each lifting task will require $\sim 2$ minutes and the whole testing protocol, including time for instrumentation, calibration and rest time should take approximately one hour and thirty minutes.

\section{$\underline{\text { Risks and Benefits of Participation: }}$}

Research has shown that manual materials handling tasks have higher risks of musculoskeletal injuries, especially low back pain. To protect you as much as possible from this risk, we will encourage you to quit at any point if you are experiencing more pain or discomfort than you would anticipate with this task. If you feel extended soreness or pain after completion of your participation, please go to a medical centre for assistance or contact your preferred health care professional.

Another possible problem for individuals with sensitive skin resulting is skin irritation from adhesive tape. Normally this irritation disappears shortly after the tape is removed. If it does not disappear within a couple of hours, please go to a medical centre for assistance.

In terms of the benefits of the study, there are no direct personal benefits expected. However, your involvement will aid in better understanding the lifting strategies of males and females with hopes of developing safe lifting practices.

\section{Confidentiality:}

All information obtained during the course of this study is strictly confidential and your anonymity will be protected at all times. Your identity will not be recorded; rather a code number will be assigned to your data. All data recorded in computer files will be locked and only the principal researcher and research assistants will be granted access. In all cases of publication, only summary data are used and this is done in such a way that no individual can be identified. 


\section{Voluntary Nature of the Study:}

As a participant, you are a volunteer who may withdraw from the study at any time without coercion or penalty. You may withdraw after hearing about the details of the study or you may also withdraw at any point during the study with no penalty.

\section{Contacts:}

If at any time you have further questions, problems or adverse events, you can contact:

Dr. Joan Stevenson (School of Kinesiology and Health Studies)

(613) 533-6288

Dr. Jean Cote (Director, School of Kinesiology and Health Studies)

(613) 533-6601

If you have any questions regarding your rights as a research participant, you can contact:

Dr. Albert Clark (Research Ethics Board, Chair)

(613) 533-6081

\section{What Does My Signature Mean?}

I am being asked to sign below. My signature indicates that:

- I have read the letter of information

- I am aware that the purpose of the study is to assess lifting technique between males and females

- I realize I can withdraw at any time without penalty or coercion

- I can contact any of the people identified in this letter if I have questions, concerns, or complaints

- I realize that my data will be kept confidential.

- By signing this consent form, I do not waive my legal rights nor release the investigator(s) and sponsors from their legal and professional responsibilities.

\section{Participant's Copy (Please sign and keep this Ethics Consent letter for your records).}

Signature of Participant

Witness
Date

Date 


\section{Researcher's Copy Ethics Consent}

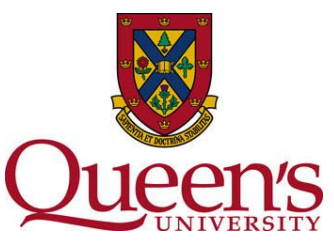

\section{Gender differences in lifting technique under increasing load conditions: A principal component analysis}

This page is for the researchers to verify that you are willing to participate in the above study. By signing this page you are declaring the following:

- You were given a verbal presentation about the above-mentioned research study

- You are aware that the purpose of the study is to assess lifting technique between males and females

- You were given an Ethics Consent letter of information to read and keep

- You realize that you can withdraw at any time without penalty or coercion

- You know that you can contact any of the people identified in the Ethics Consent letter if you have questions, concerns, or complaints

- You realize that your data will be kept confidential

- By signing this consent form, you realize that you do not waive your legal rights nor release the investigator(s) and sponsors from their legal and professional responsibilities.

$\underline{\text { (Please sign and return this page ONLY to the researchers) }}$

Print your Name

Date

Signature of Participant

Witness

Date 


\section{Appendix C Vertebral Column Anatomy and Function}

When examining lifting technique and low back disorder it is important to understand the anatomy of particular joints involved in the task. A brief review of the anatomy of the back is presented.

\section{The Vertebral Column}

The vertebral column has two main purposes: 1) it supports the trunk and, 2) protects the spinal cord. The vertebral column contains 33 vertebrae, twenty-four of which are movable and are divided among the cervical (7), thoracic (12), and lumbar (5) regions. The remaining 9 vertebrae make up the sacrum (5 fused vertebrae) and coccyx (4 fused vertebrae) (Goss, 1969; Norris, 2008). In the sagittal plane the vertebral column contains several spinal curves, which correspond with the different regions mentioned. From the dens (projection from axis or C2) to the middle of the second thoracic vertebra is a ventral convex (lordosis) curve known as the cervical curve, which is the least pronounced of the curves (Norris, 2008). The thoracic curve is concave which begins at the middle of the second thoracic vertebra and ends at the middle of the twelfth thoracic vertebra. The lumbar curve is a ventral convex curve that begins at the middle of the last thoracic vertebra and ends at the junction between the fifth lumbar vertebrae and sacrum. The lumbar curve is the most pronounced, and is usually greater in females than males (Goss, 1969). The final curve is known as the pelvic curve, which begins at the sacrovertebral articulation and ends at the point of the coccyx. The pelvic curve is directed caudally and ventrally (Goss, 1969). The vertebral column also has a slight lateral curvature, which is usually 
directed towards the right side, due to the majority of people being right hand dominant (Goss, 1969). A typical vertebra consists of two essential parts: a ventral segment, known as the vertebral body, and the dorsal aspect, known as the vertebral arch (Goss, 1969; Kalichman \& Hunter, 2007; Marras, 2008).

The vertebral body is the largest part of the vertebra. It is fairly cylindrical in shape, and its width increases from the second cervical to the first thoracic vertebra (Goss, 1969). The vertebral body is designed to resist large compressive forces and deliver nutrients to the intervertebral discs through the nutrient foramina (Adams \& Hutton, 1981; Goss, 1969). The outer portion of the vertebral body is composed of dense cortical bone, which provides strength and a high resistance to bending and torsion (Marras, 2008). Inside the vertebral body is a spongy matrix of cancellous bone, which is less dense and more elastic than cortical bone. The cancellous bone is composed of bundles of short parallel strands of bone fused together. This type of bone, along with its arrangement, allows helps the bone to maintain its shape despite compressive forces (Marras, 2008).

The vertebral arch encloses the vertebral foramen, which serves as a protective channel for the spinal cord. The vertebral arch consists of a pair of pedicles and a pair of laminae, which together support seven processes. The pedicles are two thick processes that project dorsally, one on either side of the posterior surface of the vertebral body (Ebraheim, Hassan, Lee, \& Xu, 2004). They join a pair of fused laminae, which are two broad plates directed dorsally and medially from the pedicles. The laminae fuse in the middle line to complete the dorsal part of the vertebral arch, creating the vertebral foramen (Ebraheim et al., 2004; Goss, 1969). The vertebral foramen is a triangular opening that protects the spinal cord (Adams \& Hutton, 1981). Projecting laterally at either side from the point where the lamina joins the pedicle are the transverse processes that serve as an attachment for muscles and ligaments (Goss, 1969). The spinous process is directed 
dorsally from the junction of the laminae, and also serves as an attachment for muscles and ligaments (Goss, 1969). The spinous processes are the bones that you can often see on an individual's back and, along with the transverse processes, form a guidance system for the back that keeps the bones aligned and serve to prevent the spine from excessive twisting (Marras, 2008). They also provide some load support under various conditions (Marras, 2008). Similar to the vertebral body, the posterior spinal elements also possess a hard cortical bone exterior, coupled with a cancellous bony core (Bogduk, 2005; Goss, 1969).

\section{Lumbar Vertebral Column}

\subsection{Lumbar Vertebrae}

The lumbar vertebral column is composed of five vertebrae (Adams \& Dolan, 1995; Ebraheim et al., 2004; Goss, 1969). The lumbar vertebrae are the largest segments of the movable part of the vertebral column (Goss, 1969). They are different from the other vertebrae in that they do not contain a foramen in the transverse process, and by the absence of facets for the ribs, on the sides of the body (Goss, 1969). The body is large, wider transversely and slightly thicker ventrally (Goss, 1969). The vertebral foramen is once again triangular, larger than in the thoracic vertebrae, but smaller than in the cervical region (Goss, 1969). The spinous process is thick, broad, and quadrilateral, ending in a rough uneven border (Goss, 1969). The fifth lumbar vertebra has a body that is much deeper ventrally than dorsally, which is due to the lumbo-sacral articulation. The laminae are broad, short, and strong. Inferior and superior articular processes extend from each lamina, and there is a smooth area of bone on the medial and lateral surfaces of the superior and inferior articular processes, respectively (Bogduk, 2005). These smooth areas are known as articular facets (Bogduk, 2005; Goss, 1969). 


\subsection{Articulations}

When two lumbar vertebrae are placed together, three articulations (or joints) are made (Ebraheim et al., 2004; Hollinshead, 1969; Norris, 2008). The articulations include the intervertebral disc anteriorly and a pair of the facet, or zygapophyseal, joints posteriorly (Ebraheim et al., 2004; Hickey \& Hukins, 1980). These joints are given further support from various ligaments. The intervertebral discs are located between adjacent vertebral bodies and bind the vertebrae together while permitting the series of alternate discs and bones to function as a flexible and resilient supporting column for the body (Hickey \& Hukins, 1980). According to Marras (2008), the intervertebral discs serve three main purposes. First they serve as shock absorbers, second they transmit load, and third they permit motion between the vertebrae (Marras, 2008). The motions they allow are flexion, extension, and lateral bending motions (Ebraheim et al., 2004).

Invertebral discs are avascular and consist of a nucleus pulposus centre, encircled by the annulus fibrosus. The nucleus pulposus is composed of a mucoid material, which contains $70 \%$ to 90\% water (Adams \& Dolan, 1995; Ebraheim et al., 2004). The water content in the nucleus pulposus gradually decreases with age, which results in disc degeneration (Ebraheim et al., 2004; Norris, 2008). Because of its high water content, coupled with collagen fibrils, and a highly hydrated gel known as glycosaminoglycans, the nucleus pulposus behaves like a pressurized fluid that is virtually incompressible (Adams \& Dolan, 1995; Ebraheim et al., 2004; Hickey \& Hukins, 1980).

The annulus fibrosus contains layers of obliquely-oriented fibrous tissue arranged in concentric rings (approximately 20), called lamellae that overlap each other and are arranged like those in an onion (Ebraheim et al., 2004; Norris, 2008). The annulus fibrosus consists mainly of collagen fibers (15\%), tendon (30\%), and a reasonable portion of glycosaminoglycan (Hickey \& 
Hukins, 1980). It looks like a laminated structure surrounding the nucleus pulposus (Ebraheim et al., 2004). Collagen fibers in individual lamellae of the annulus fibrosus are parallel and tilted with respect to the spine (Hickey \& Hukins, 1980). The tilt angle of approximately 65 degrees alternates in successive lamellae (Hickey \& Hukins, 1980). The posterior portion of the annulus fibrosus is thinner as compared with its anterior portion (Ebraheim et al., 2004). The peripheral fibers of the annulus fibrosus insert into the cartilaginous end plates and anterior and posterior longitudinal ligaments. In the posterior portion of the disc, the fibers run in a more vertical direction.

The facet joint, also known as the zygapophyseal joint, are true synovial joints that consists of the posterolateral articulation between vertebral levels (Kalichman \& Hunter, 2007). Each joint consists of a superior and an inferior articular process. The superior articular process is a large, posteriorly, and medially facing concave surface from the inferior vertebral level. The inferior articular process is an anteriorly and laterally facing process from the superior vertebral level (Kalichman \& Hunter, 2007). The morphology of each joint is somewhere between a C and a J shape (Bogduk, 2005) (Figure 12). 

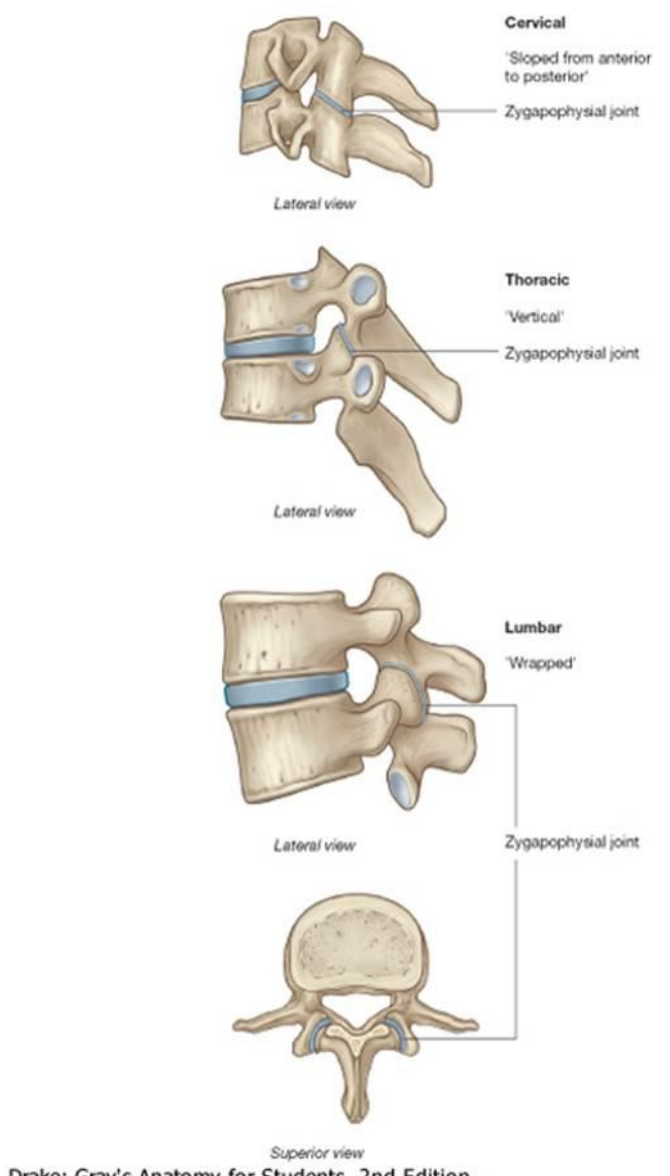

Drake: Gray's Anatomy for Students, 2nd Edition.

Copyright (c) 2009 by Churchill Livingstone, an imprint of Elsevier, Inc. All rights reserved.

Figure 11. The orientation of facet joints in the cervical, thoracic, and lumbar vertebrae (Drake, 2010)

Lumbar facet joints contain hyaline cartilage, synovial membrane, fibrous capsule, and a small joint space (Kalichman \& Hunter, 2007). Lumbar facet joints also contain menisci that serve to compensate for the incongruence of articular surfaces and to fill in empty spaces (Kalichman \& Hunter, 2007; Kos, Hert, \& Sevcik, 2002). Panjabi et al. (1993) reported that the menisci as well as the facet area increase in size from L1-L2 to L5-S1. The orientation of facet joints can be important in providing stability to the spine and controlling its motion under various loads (Kalichman \& Hunter, 2007). The lumbar facets are sagitally oriented with those of T12 to 
L2 oriented closer to the midsagittal plane of the vertebral body (mean range, 26-34 ), and the facets of L3 to L5 oriented away from that plane (mean range, 40-56 ${ }^{\circ}$ (Masharawi et al., 2004). The oblique orientation of the facet joints aids in the resistance to intervertebral shear forces, compressive forces, and intervertebral torsion (Adams \& Hutton, 1981). The sagittal orientation of the facet joints allows for greater range of flexion and limits axial rotation in the lumbar spine. The facet joints are important for load transmission, mostly by providing posterior load-bearing structures, and for stabilizing flexion and extension motions (Kalichman \& Hunter, 2007). In addition, facet joints are important because they resist axial rotation and forward sliding of the lumbar vertebrae (Kalichman \& Hunter, 2007).

\subsection{Ligaments}

A number of ligaments are critical to the stabilization of the vertebral column as one unit (Ebraheim et al., 2004). The main ligaments are the anterior and posterior longitudinal ligaments, ligamentum flavum, supraspinous ligament, and interspinous ligament (Ebraheim et al., 2004). The anterior longitudinal ligament is a strong band that attaches to the whole anterior aspect of the vertebral bodies and intervertebral discs from the skull down to the upper part of the sacrum (Ebraheim et al., 2004; Marras, 2008). The antero-medial aspect of the anterior longitudinal ligament is the thickest portion. The superficial fibers are the longest and extend over 3 to 4 vertebrae, whereas the deepest fibers extend over 2 vertebrae and are firmly attached to the inferior margin of one vertebrae and the superior margin of the next (Ebraheim et al., 2004). The main function of the anterior longitudinal ligament is to limit extension of the spinal column (Norris, 2008).

The posterior longitudinal ligament is the main ligament that opposes the anterior longitudinal ligament. It attaches to the posterior aspect of the vertebral bodies and discs, from 
the occipital bone to the sacrum (Ebraheim et al., 2004; Vleeming, Mooney, Snijders, Dorman, \& Stoeckart, 1999). In the cervical region, it is broad and uniform, whereas in the thoracic and lumbar regions it is narrow over the middle of the vertebrae and broad over the discs (Ebraheim et al., 2004). In the intervertebral foramen, the posterior longitudinal ligament extends laterally and fuses with the lateral extensions of the anterior longitudinal ligament (Ebraheim et al., 2004). As with the anterior longitudinal ligament, the superficial fibers of the posterior longitudinal ligament extend over three to four vertebrae, and the deep fibers attach two adjacent vertebrae (Ebraheim et al., 2004). The role of the posterior longitudinal ligament is to stabilize the spinal column during flexion (Adams \& Dolan, 1995; Ebraheim et al., 2004; Norris, 2008) .

The ligamentum flavum is a short, thick ligament present between the lamina of adjacent vertebrae (Ebraheim et al., 2004; Yahia, Garzon, Strukowski, \& Rivard, 1990). It is composed mainly of elastic fibers (80\%) which run in a vertical direction (Ebraheim et al., 2004; Yahia et al., 1990). The ligamentum flavum extends from the lower portion of the anterior surface of the upper laminae to the upper portion of the posterior surface of the lower laminae (Ebraheim et al., 2004). As a result, it covers the entire interlaminar space (Ramsey, 1966). Laterally, the ligamentum flavum fuses with the capsule of the facet joint. It has two layers: one superficial and one deeper. The function of the ligamentum flavum is to resist trunk flexion (Gillespie \& Dickey, 2004).

The interspinous and supraspinous ligaments connect the spinous processes with each other. The interspinous ligament is thin and extends from the lower border of one spinous process to the upper border of the next (Ebraheim et al., 2004). The supraspinous ligament runs over the spinous processes from the occipital bone to the sacrum, and is stronger than the interspinous ligament (Ebraheim et al., 2004; Vleeming \& Pool-Goudzwaard, 1995; Vleeming et al., 1999). The interspinous and supraspinous ligaments act to resist flexion movements. They provide more 
resistance with greater degrees of flexion (Adams \& Dolan, 1995). The intertransverse ligaments are located between the transverse processes.

Ligaments provide support for the spine. However, this support is passive, meaning that they only provide support when they are under tension, which usually occurs when the spine is in a non-neutral or deviated posture (Marras, 2008; Vleeming et al., 1999). It is important to note that, although the ligaments of the vertebral column have been described as discrete structures, in reality they are merged at their edges and act functionally as a single unit (Norris, 2008; Vleeming et al., 1999). Studies have also shown that ligaments are capable of providing feedback to the musculoskeletal system and can actually initiate muscle activities if they are excessively stretched (Marras, 2008; Solomonow, 2004).

\subsection{Muscles of the Low Back}

There are over thirty muscles that support and load the vertebral column (Marras, 2008). The muscles surrounding the lumbar spine can be distinguished and separated into three main groups based on their location. The posterior muscles of the lumbar spine can be further separated into superficial, intermediate, and deep layers. The superficial layer is the thoracolumbar fascia which will be discussed in further detail later. The intermediate layer consists of the serratus posterior inferior, which originates from the spinous processes of the cervicothoracic and thoracolumbar spine and inserts into the ribs (Vleeming et al., 1999). The deep layer consists of the erector spinae muscles, which are vertically oriented bundles of muscle that originate from the iliosacrolumbar region up to the cervical region (Ebraheim et al., 2004). The three columns of erector spinae muscles under the thoracolumbar fascia are: iliocostalis, longissimus, and spinalis, which are oriented laterally, centrally, and medially, respectively (Ebraheim et al., 2004). The iliocostalis is further divided into iliocostalis lumborum, ilicostalis thoracis, and iliocostalis 
cervicis based on their distribution. It originates from the posterior iliac crest and has insertion points on the ribs and the transverse processes of the lower cervical spine (Ebraheim et al., 2004; White \& Panjabi, 1990).

The largest muscle of the erector spinae is the longissimus, and it too is divided into three units known as: longissimus thoracis, longissimus cervicis, and longissimus capitis (Ebraheim et al., 2004). The longissimus originates from the posterior surface of the sacrum and has insertion points on the transverse processes of the thoracic and cervical spine, as well as the mastoid process (Ebraheim et al., 2004). The smallest of the erector spinae muscles is the spinalis. Similar to the others, the spinals has three divisions. The spinalis thoracic, spinalis cervicis, and spinalis capitis originate from the spinous processes of the upper lumbar spine and attach to the spinous processes on the vertebrae that are superior (Ebraheim et al., 2004). There are several short muscles under the erector spinae called semispinalis, multifidus, and rotatores. They are obliquely oriented between the spinous processes and the transverse processes of the spine (Ebraheim et al., 2004). The functions of the posterior muscles of the lumbar spine include extension, lateral bending, and rotation (Norris, 2008).

The antero-lateral muslces of the lumbar spine include the psoas major and quadratus lumborum (Ebraheim et al., 2004). The psoas major originates from the anterolateral aspect of the vertebral bodies and inserts onto the lesser trochanter of the femur (Ebraheim et al., 2004). The quadratus lumborum originates from the L5 transverse process and iliac crest and inserts onto the transverse processes above the twelfth rib (Ebraheim et al., 2004). Flexion and spinal rotation are the main functions of the anterior and lateral lumbar spine muscles (Norris, 2008).

Since the muscles surrounding the lumbar spine are located both anteriorly and posteriorly, they provide a great deal of stability and movement. Muscles provide active support to the lumbar vertebral column, meaning that the muscles generate force and are activated in response to external loads, such as lifting. However, the muscles of the lumbar spine are at a 
mechanical disadvantage compared to the external loads due to the short lever arm relative to the spine (Marras, 2008). As a result, the muscles surrounding the lumbar vertebral column are capable of producing extremely large forces on the spine.

\subsection{Fascia}

Other important support mechanisms for the vertebral column are the fascia. A fascia is a sheet of fibrous connective tissue that envelops, separates, or binds soft tissue such as muscles and organs (Marras, 2008). The fascia that lies over the musculature of the lumbar spine is known as the thoracolumbar fascia; it provides support and transmits force along the vertebral column (Marras, 2008). The thoracolumbar fascia can be separated into three layers that envelop the muscles (Marras, 2008). First is a thin anterior layer that is attached to the quadratus lumborum. The intermediate layer is posterior to the quadratus lumborum and is attached to the transverse processes of the lumbar vertebrae. The posterior thoracolumbar fascia surrounds the back muscles. It originates at the spinous processes and wraps around the muscles of the vertebral column and attaches to the thoracolumbar fascia along the lateral portion of the iliocostalis lumborum (Marras, 2008).

\subsection{Nerves of the Lumbar Spine}

The spinal cord lies within the vertebral canal and is separated from the vertebrae and its

connecting ligaments by a layer of fatty connective tissue and special coverings known as the meninges (Hollinshead, 1969). The spinal nerves branch from the spinal cord and make their exit between adjacent vertebrae in the lumbosacral region, usually through the intervertebral foramina 
(Ebraheim et al., 2004; Hollinshead, 1969). There are 11 pairs of spinal nerves in the lumbar region. These include 5 lumbar, 5 sacral, and 1 coccygeal. The spinal nerves further divide into a small dorsal ramus and a large ventral ramus once it has exited from the intervertebral foramina (Ebraheim et al., 2004). The dorsal ramus runs posteriorly and supplies the spinal ligaments, as well as the muscles and skin of the back. The ventral rami are longer and run in an infero-lateral direction in the lumbar region to form the lumbar and sacral plexuses (Ebraheim et al., 2004). The plexuses give rise to nerves supplying the muscles, joints, and skin of the upper and lower abdomen (Ebraheim et al., 2004).

The lumbar plexus is a combination of the ventral rami of the L1, L2, L3, and L4 nerves (Ebraheim et al., 2004). It runs in the infero-lateral direction, passes posterior to the psoas major muscle and anterior to the quadratus lumborum muscle. It supplies part of the lower extremity and the lower abdominal wall. The largest branch from the lumbar plexus is the femoral nerve, which passes under the inguinal ligament and supplies the flexor muscles of the hip and the extensor muscles of the knee (Ebraheim et al., 2004). The ventral rami of L4 to S4 nerves are contained within the sacral plexus. The sacral plexus supplies the buttocks, perineum, and lower extremities. The sciatic nerve is the largest branch from the sacral plexus, which supplies the entire leg and foot (Ebraheim et al., 2004).

\subsection{Blood Supply}

Blood delivery is important to maintain the health of biologic tissues, and is involved in the perception of pain (Marras, 2008). The lumbar spine blood supply is provided by four pairs of lumbar arteries, which arise from the back of the aorta in front of L1 to L4, as well as a fifth pair that arises from the median sacral artery (Ratcliffe, 1980). At the intervertebral foramen, the 
lumbar artery enters the spinal canal where it is then termed the spinal artery (Ratcliffe, 1980). The spinal artery divides into an ascending and descending branch, deep to the posterior longitudinal ligament (Ratcliffe, 1980). The branches then anastomose with the spinal arteries above and below to form a longitudinal series of arteries (Ebraheim et al., 2004). The blood supply to the vertebrae, spinal cord and cauda equina is provided by branches from the spinal artery (Ebraheim et al., 2004). The main blood supply of the spinal cord is provided by a single anterior spinal artery, along with posterior spinal arteries and several radicular arteries (Ebraheim et al., 2004; Ratcliffe, 1980).

The anterior internal vertebral venous plexus and posterior internal vertebral venous plexus are veins that surround the spinal cord in the epidural space. The anterior internal venous plexus is composed of two longitudinal veins located between the pedicles and the posterior longitudinal ligament (Ebraheim et al., 2004). Similar to the arteries, the veins anastomose with each other and drain blood from the vertebral body (Ebraheim et al., 2004). The blood from the internal venous plexus is drained through the intervertebral foramen into the segmental veins and into the inferior vena cava (Bogduk, 2005; Ebraheim et al., 2004). 


\section{References}

Adams, M. A., \& Dolan, P. (1995). Recent advances in lumbar spinal mechanics and their clinical significance. Clinical Biomechanics, 10(1), 3-19.

Adams, M. A., \& Hutton, W. C. (1981). The relevance of torsion to the mechanical derangement of the lumbar spine. Spine, 6(3), 241-248.

Bogduk, N. (2005). Clinical anatomy of the lumbar spine and sacrum / (4th ed. ed.). Edinburgh; New York: Elsevier/Churchill Livingstone.

Drake, R. L., Vogl, W., \& Mitchell, A. W. M. (2010). Gray's anatomy for students / (2nd ed. ed.). Edinburgh: Churchill Livingstone.

Ebraheim, N. A., Hassan, A., Lee, M., \& Xu, R. (2004). Functional anatomy of the lumbar spine. Seminars in Pain Medicine, 2(3), 131-137.

Gillespie, K., \& Dickey, J. (2004). Biomechanical role of lumbar spine ligaments in flexion and extension: Determination using a parallel linkage robot and a porcine model. Spine, 29(11), $1208-1216$.

Goss, C. M. (Ed.). (1969). Gray's anatomy (28th ed.). Philadelphia: Lea \& Febiger.

Hickey, D., \& Hukins, D. (1980). Relation between the structure of the annulus fibrosus and the function and failure of the intervertebral dics. Spine, 5, 100-116.

Hollinshead, W. H. (1969). Functional anatomy of the limbs and back (2nd ed.). Philadelphia, PA: W.B. Saunders Company. 
Kalichman, L., \& Hunter, D. J. (2007). Lumbar facet joint osteoarthritis: A review. Seminars in Arthritis and Rheumatism, 37(2), 69-80.

Kos, J., Hert, J., \& Sevcik, P. (2002). Meniscoids of the intervertebral joints. Acta Chirurgiae Orthopaedicae et Traumatologiae Cechoclovaka, 69, 149-157.

Marras, W. (2008). Working back : A systems view /. Hoboken, N.J.: Wiley-Interscience.

Masharawi, Y., Rothschild, B., Dar, G., Pleg, S., Robinson, D., \& Breen, E. (2004). Facet orientation in the thoracolumbar spine: Three-dimensional anatomic and biomechanical analysis. Spine, 29, 1755-1763.

Norris, C. M. (Ed.). (2008). Back stability (2nd ed.). Champaign, IL: Human Kinetics.

Panjabi, M., Oxland, T., Takata, K., Goel, V., Duranceau, J., \& Krag, M. (1993). Articular facets of the human spine: Quantitative three-dimensional anatomy. Spine, 18, 1298-1310.

Ramsey, R. H. (1966). The anatomy of the ligamenta flava. Clinical Orthopedics, 44, 129-140.

Ratcliffe, J. (1980). The arterial anatomy of the adult human vertebral body: A microarteriographic study. Journal of Anatomy, 131, 57-79.

Solomonow, M. (2004). Ligaments: A source of work-related musculoskeletal disorders. Journal of Electromyography and Kinesiology, 14, 49-60.

Vleeming, A., Mooney, V., Snijders, C. J., Dorman, T. A., \& Stoeckart, R. (1999). Movement, stability and low back pain. London, UK: Churchill Livingstone. 
Vleeming, A., \& Pool-Goudzwaard, A. L. (1995). The posterior layer of the thoracolumbar fascia. Spine, 20, 753-758.

White, A., \& Panjabi, M. (1990). Clinical biomechanics of the spine (2nd ed.). Philadelphia, NY: J.B. Lippincott Company.

Yahia, L., Garzon, S., Strukowski, H., \& Rivard, C. (1990). Ultrastructure of the human interspinous ligament and ligamentum flavum: A preliminary study. Spine, 15(4), 262-268. 


\section{Appendix D \\ Principal Component Analysis}

The main reason for using principal components analysis (PCA) is to summarize the most important information in the data (Deluzio, Wyss, Zee, Costigan, \& Serbie, 1997; Deluzio, Wyss, Costigan, Sorbie, \& Zee, 1999; Deluzio \& Astephen, 2007; Khalaf, Parnianpour, Sparto, \& Barin, 1999; Nguyen \& Reynolds, 2009; Sadler, Graham, \& Stevenson, 2011; Wrigley, Albert, Deluzio, \& Stevenson, 2005; Wrigley, Albert, Deluzio, \& Stevenson, 2006). PCA is a quantitative method for achieving both data reduction and separating useful parameters from redundant ones (Nguyen \& Reynolds, 2009). This is accomplished by representing the observations and the variables simultaneously in a limited number of optimal principal components (PCs) or features (Deluzio \& Astephen, 2007). The features are optimal in that they explain a maximal amount of variance in the original data set (Deluzio \& Astephen, 2007).

The process involves creating matrices of waveforms, such that every participant's

waveform of data are entered as a row vector $\boldsymbol{X} \boldsymbol{x} \boldsymbol{p}$ representing $n$-observations (waveform) and $p$-variables (normalized time points) (Wrigley et al., 2006). The matrices are as follows:

$$
\left[\begin{array}{cccc}
x_{1,1} & x_{1,2} & \ldots & x_{1, p} \\
x_{2,1} & x_{2,2} & \ldots & x_{2, p} \\
\vdots & \vdots & \ddots & \vdots \\
x_{n, 1} & x_{n, 2} & \cdots & x_{n, p}
\end{array}\right]
$$

PCA consists of an orthogonal transformation that converts $p$ variables $\boldsymbol{X}=\boldsymbol{x}_{\mathbf{1}}, \boldsymbol{x}_{2}, \ldots \boldsymbol{x}_{\boldsymbol{p}}$ into $z$ new uncorrelated principal components $\boldsymbol{Z}=\boldsymbol{z}_{\mathbf{1}}, \boldsymbol{z}_{\mathbf{2}}, \ldots \boldsymbol{z}_{\boldsymbol{p}}$ (Deluzio \& Astephen, 2007). The principal components are mutually uncorrelated in the sample and are arranged in decreasing 
order in their sample (Deluzio et al., 1997; Deluzio \& Astephen, 2007). That is, the first principal component explains the largest amount of variance, and subsequent PCs describe less variance in the original data set (Khalaf et al., 1999). The principal component model is $\boldsymbol{Z}=\boldsymbol{U}^{\boldsymbol{t}} \boldsymbol{X}$ where the columns of $\boldsymbol{U}=\boldsymbol{u}_{1}, \boldsymbol{u}_{2}, \ldots \boldsymbol{u}_{p}$ are called principal component loading vectors, and are the eigenvectors of the covariance matrix of $\boldsymbol{X}$ (Deluzio \& Astephen, 2007). Since the eigenvector matrix is orthonormal, the original dataset can be reconstructed by inverting the principal component model $\boldsymbol{X}=\boldsymbol{U Z}$ (Deluzio \& Astephen, 2007).

The principal component loading vectors, $\boldsymbol{u}_{\boldsymbol{i}}$, are an orthogonal basis set for the waveform that represent specific features of the data (Deluzio \& Astephen, 2007). The principal component score, $\boldsymbol{z}_{\boldsymbol{i}}$, correspond to the contribution of the principal components to each individual waveform (Deluzio \& Astephen, 2007). Therefore, each individual participant's original waveform is transformed into a set of PC scores that measure the degree to which the shape of their waveform corresponds to each feature.

The advantage of PCA is that if the majority of the variation is explained by the first few principal components, resulting in $k<p$, the remaining PCs can be dropped and data reduction is accomplished (Deluzio et al., 1997). From previous research it has been deemed that a $90 \%$ trace criteria is adequate to describe the majority of the variation in the data, and capture the primary modes of variation (Deluzio \& Astephen, 2007).

Interpretation of PCA is achieved through visual inspection of the PC loading vector, as well as waveforms of participants that scored low $\left(5^{\text {th }}\right.$ percentile) and high $\left(95^{\text {th }}\right.$ percentile $)$ on each PC (Astephen, Deluzio, Caldwell, Dunbar, \& Hubley-Kozey, 2008; Deluzio \& Astephen, 2007; Jones \& Rice, 1992; Reid, Graham, \& Costigan, 2010; Sadler et al., 2011). According to Wrigley et al. (2006), the pattern of variation can often be described by one of three common operators. The first is a magnitude operator which describes variation in the waveform amplitudes over the entire lift time or within a specific region. The second is a difference operator which 
describes a change from either having a relatively low to high waveform amplitude. Finally, the third is a phase shift operator which captures a change in the relative timing of waveform events. 


\section{References}

Astephen, J. L., Deluzio, K. J., Caldwell, G., Dunbar, M. J., \& Hubley-Kozey, C. L. (2008). Gait and neuromuscular pattern changes are associated with differences in knee osteoarthritis severity levels. Journal of Biomechanics, 41, 868-876.

Deluzio, K.J., \& Astephen, J. L. (2007). Biomechanical features of gait waveform data associated with knee osteoarthritis: An application of principal component analysis. Gait and Posture, $25,86-93$

Deluzio, K.J., Wyss, U. P., Costigan, P. A., Sorbie, C., \& Zee, B. (1999). Gait assessment in unicompartmental knee arthroplasty patients: Principal component modelling of gait waveforms and clinical status. Human Movement Science, 18(5), 701-711.

Deluzio, K.J., Wyss, U. P., Zee, B., Costigan, P. A., \& Serbie, C. (1997). Principal component models of knee kinematics and kinetics: Normal vs. pathological gait patterns. Human Movement Science, 16(2-3), 201-217.

Jones, M. C., \& Rice, J. A. (1992). Displaying the important features of large collections of similar curves. The American Statistician, 46(2), pp. 140-145.

Khalaf, K. A., Parnianpour, M., Sparto, P. J., \& Barin, K. (1999). Feature extraction and quantification of the variability of dynamic performance profiles due to the different sagittal lift characteristics. Rehabilitation Engineering, Institute of Electrical and Electronics Engineers IEEE Transactions on, 7(3), 278-288. 
Nguyen, T. C., \& Reynolds, K. J. (2009). Principal component analysis of lifting kinematics and kinetics in pregnant subjects. International Conference on Biomedical Engineering (ICBME), 23, 1888-1891.

Reid, S., Graham, R., \& Costigan, P. (2010). Differentiation of young and older adult stair climbing gait using principal component analysis. Gait and Posture, 31, 197-203.

Sadler, E. M., Graham, R. B., \& Stevenson, J. M. (2011). Gender difference and lifting technique under light load conditions: A principal component analysis. Theoretical Issues in Ergonomics Science, , 1-16.

Wrigley, A., Albert, W., Deluzio, K., \& Stevenson, J. (2005). Differentiating lifting technique between those who develop low back pain and those who do not. Clinical Biomechanics, 20, 254-263.

Wrigley, A., Albert, W., Deluzio, K., \& Stevenson, J. (2006). Principal component analysis of lifting waveforms. Clinical Biomechanics, 21, 567-578. 


\section{Appendix E \\ Principal Component Elimination}

Principal component analysis (PCA) is an effective method to achieve data reduction and analysis of waveforms (Deluzio, Wyss, Zee, Costigan, \& Serbie, 1997; Khalaf, Parnianpour, Sparto, \& Barin, 1999; Nguyen \& Reynolds, 2009). The interpretation of principal components (PCs) is achieved through visual inspection of waveforms of participants who score high and low (Astephen, Deluzio, Caldwell, Dunbar, \& Hubley-Kozey, 2008; Deluzio \& Astephen, 2007; Jones \& Rice, 1992; Reid, Graham, \& Costigan, 2010; Sadler, Graham, \& Stevenson, 2011). This method is both difficult and subjective. Since the amount of variance explained decreases with each subsequent PC, the interpretation of PCs becomes increasingly difficult. This is due to the fact that the raw waveforms used for visual inspection contain features associated with the preceding PC (Sadler et al., 2010). In order to enhance visual inspection of PCs, features associated with the preceding PC should be removed from the raw waveforms of the individuals who score in the $5^{\text {th }}$ and $95^{\text {th }}$ percentile.

Principal component elimination (PCE) is a method developed by Sadler, (2010) to remove features associated with preceding PCs from the raw waveforms in order to enhance interpretability. To help interpret a specific PC $\left(P C_{n}\right)$, with $k$ PCs in the model, the individual's $P C_{n-1}$ to $P C_{n-(k-1)}$ are eliminated (Sadler, 2010). A flow chart describing the process is

presented in Figure 12. First, the individuals who score high $\left(95^{\text {th }}\right.$ percentile $)\left(x_{H}\right)$ and low $\left(5^{\text {th }}\right.$ percentile) $\left(x_{L}\right)$ on $P C_{n}$ are identified. Second, PC1 through to the preceding PC in question $\left(P C_{n-(k-1)}\right)$ for these individuals are multiplied by the corresponding loading vector. This creating a waveform $\left(W_{P C n}\right)$ that represents the preceding PC's contribution to the individual's $\left(x_{H}\right.$ and $\left.x_{L}\right)$ original waveform $\left(W_{o}\right)$ (Sadler, 2010). This waveform $\left(W_{P C n}\right)$ is then subtracted 
Identify participants who scored High and Low on the PC to be analyzed
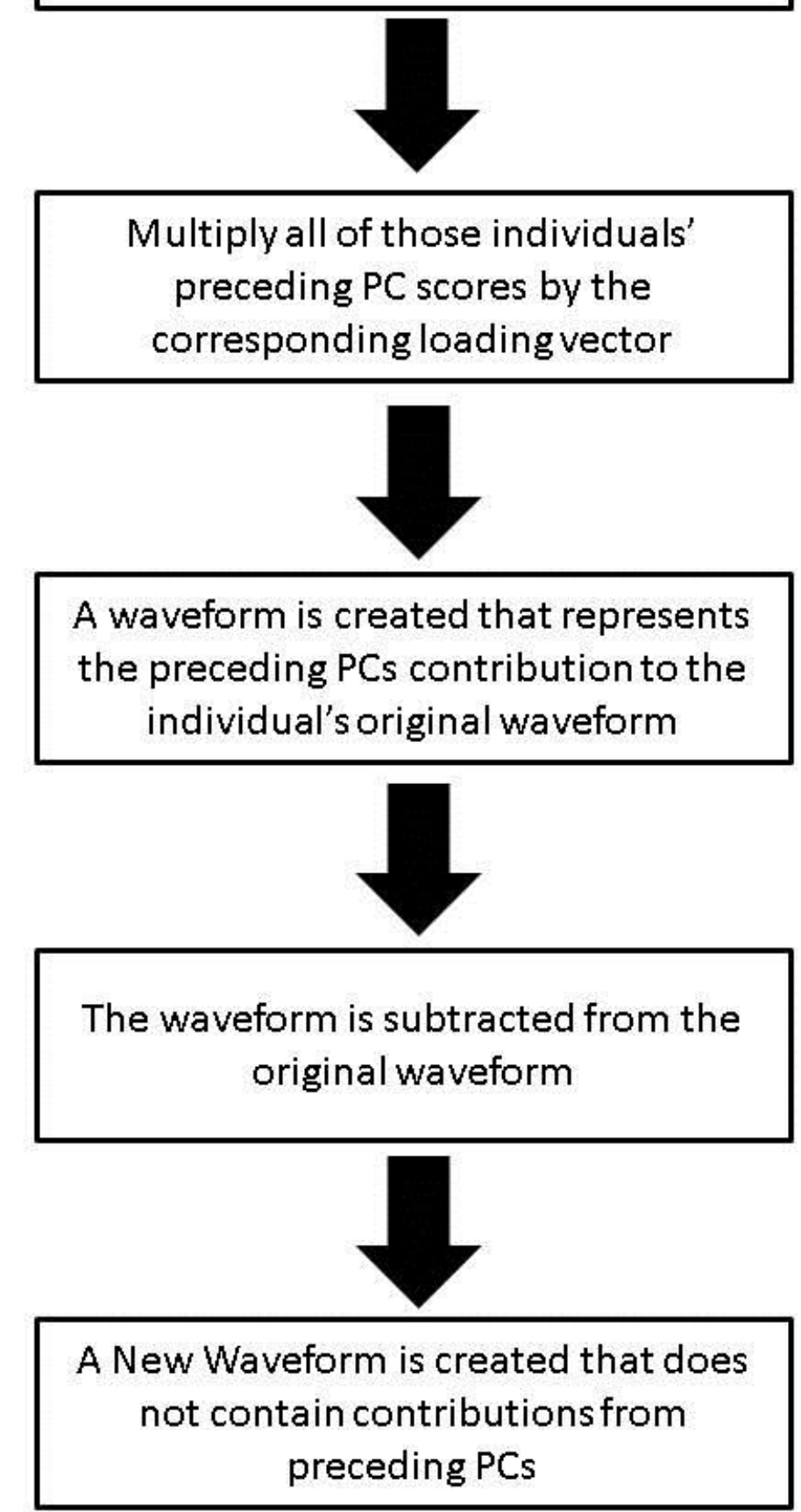

Figure 12. Flow chart describing principal component elimination (PCE). 
from the original waveform $\left(W_{o}\right)$, resulting in a new waveform $\left(W_{2}\right)$ that no longer has information of the preceding PCs.

If 2 PCs are retained for a waveform in accordance with the $90 \%$ trace criterion, the contribution of PC1 to the raw waveforms needs to be eliminated. Therefore, $W_{P C n}$ is subtracted from $W_{o}$, resulting in a new waveform $\left(W_{2}\right)$ (equation $1 \mathrm{a}$ and $1 \mathrm{~b}$ ). The new waveform is no longer contains contributions from PC1 and can be used as the new waveform for $x_{H}$ and $x_{L}$.

$$
\begin{aligned}
& W_{o_{x_{H}}}-W_{P C 1_{x_{H}}}=W_{2_{H}} \\
& W_{o_{x_{L}}}-W_{P C 1_{x_{L}}}=W_{2_{L}}
\end{aligned}
$$

For models with more than 2 PCs retained, the process is the same but with multiple iterations. Therefore, all of the preceding PC scores of those who score high and low are multiplied by their respective PC loading vectors.

As an example from the present study, hip flexion demonstrated a significant difference for three PCs. Therefore, in order to interpret PC2, PC1 was eliminated; and for PC3, PC1 and PC2 needed to be eliminated. Cases that scored high and low for PC2 were 73 (participant 23 for 30\% max load condition) and 3 (participant 3 for 10\% max load condition), respectively. Multiplication of $P C 1_{73}$ and $P C 1_{3}$ each by $P C 1$ loading vector results in equation $2 \mathrm{a}$ and $2 \mathrm{~b}$. The PCE altered waveforms to aid in the interpretability of PC2 are $W_{2_{73}}$ and $W_{2_{3}}$ (equation $3 \mathrm{a}$ and $3 b)$.

$$
\begin{aligned}
& P C 1_{73} \times P C 1_{\text {loading vector }}=W_{P C 1_{73}} \\
& P C 1_{3} \times P C 1_{\text {loading vector }}=W_{P C 1_{3}} \\
& W_{O_{73}}-W_{P C 1_{73}}=W_{2_{73}}
\end{aligned}
$$




$$
W_{o_{3}}-W_{P C 1_{3}}=W_{2_{3}}
$$

For PC3, both PC1 and PC2 must be eliminated from the raw data. Cases that scored high and low on PC3 were identified as 25 and 4 , respectively. $P C 1_{25}$ score and $P C 1_{4}$ were each multiplied by PC1 loading vector resulting in $W_{P C 1_{25}}$ and $W_{P C 1_{4}}$, respectively. Next, $P C 2_{25}$ score and $\mathrm{PC}_{4}$ were each multiplied by $\mathrm{PC} 2$ loading vector yielding $W_{P C 2_{25}}$ and $W_{P C 2_{4}}$, respectively. Using an iterative version of equation $2 \mathrm{a}$ and $2 \mathrm{~b}$, the new adjusted waveforms for PC3 interpretation were created resulting in equations $4 \mathrm{a}$ and $4 \mathrm{~b}$ :

$$
\begin{aligned}
& W_{o_{25}}-W_{P C 1_{25}}-W_{P C 2_{25}}=W_{3_{25}} \\
& W_{o_{4}}-W_{P C 1_{4}}-W_{P C 2_{4}}=W_{3_{4}}
\end{aligned}
$$




\section{References}

Astephen, J. L., Deluzio, K. J., Caldwell, G., Dunbar, M. J., \& Hubley-Kozey, C. L. (2008). Gait and neuromuscular pattern changes are associated with differences in knee osteoarthritis severity levels. Journal of Biomechanics, 41, 868-876.

Deluzio, K.J., \& Astephen, J. L. (2007). Biomechanical features of gait waveform data associated with knee osteoarthritis: An application of principal component analysis. Gait and Posture, $25,86-93$

Deluzio, K.J., Wyss, U. P., Zee, B., Costigan, P. A., \& Serbie, C. (1997). Principal component models of knee kinematics and kinetics: Normal vs. pathological gait patterns. Human Movement Science, 16(2-3), 201-217.

Jones, M. C., \& Rice, J. A. (1992). Displaying the important features of large collections of similar curves. The American Statistician, 46(2), pp. 140-145.

Khalaf, K. A., Parnianpour, M., Sparto, P. J., \& Barin, K. (1999). Determination of the effect of lift characteristics on dynamic performance profiles during manual materials handling tasks. Ergonomics, 42(1), 126-145.

Nguyen, T. C., \& Reynolds, K. J. (2009). Principal component analysis of lifting kinematics and kinetics in pregnant subjects. International Conference on Biomedical Engineering (ICBME), 23, 1888-1891.

Reid, S., Graham, R., \& Costigan, P. (2010). Differentiation of young and older adult stair climbing gait using principal component analysis. Gait and Posture, 31, 197-203. 
Sadler, E.M. (2010). Principal component analyses of joint angle curves to examine lifting technique. (Thesis). Queen's University, Kingston, Ontario, Canada.

Sadler, E. M., Graham, R. B., \& Stevenson, J. M. (2011). Gender difference and lifting technique under light load conditions: A principal component analysis. Theoretical Issues in Ergonomics Science, , 1-16. 


\begin{abstract}
Appendix F
Principal Components Retained According to the 90\% Trace Criterion
\end{abstract}


Table 7. Means, standard deviations, and p values for males and females for all principal components (PCs) retained according to the $90 \%$ trace criterion. A p value of $\mathrm{p}=0.005$ represented a significant difference

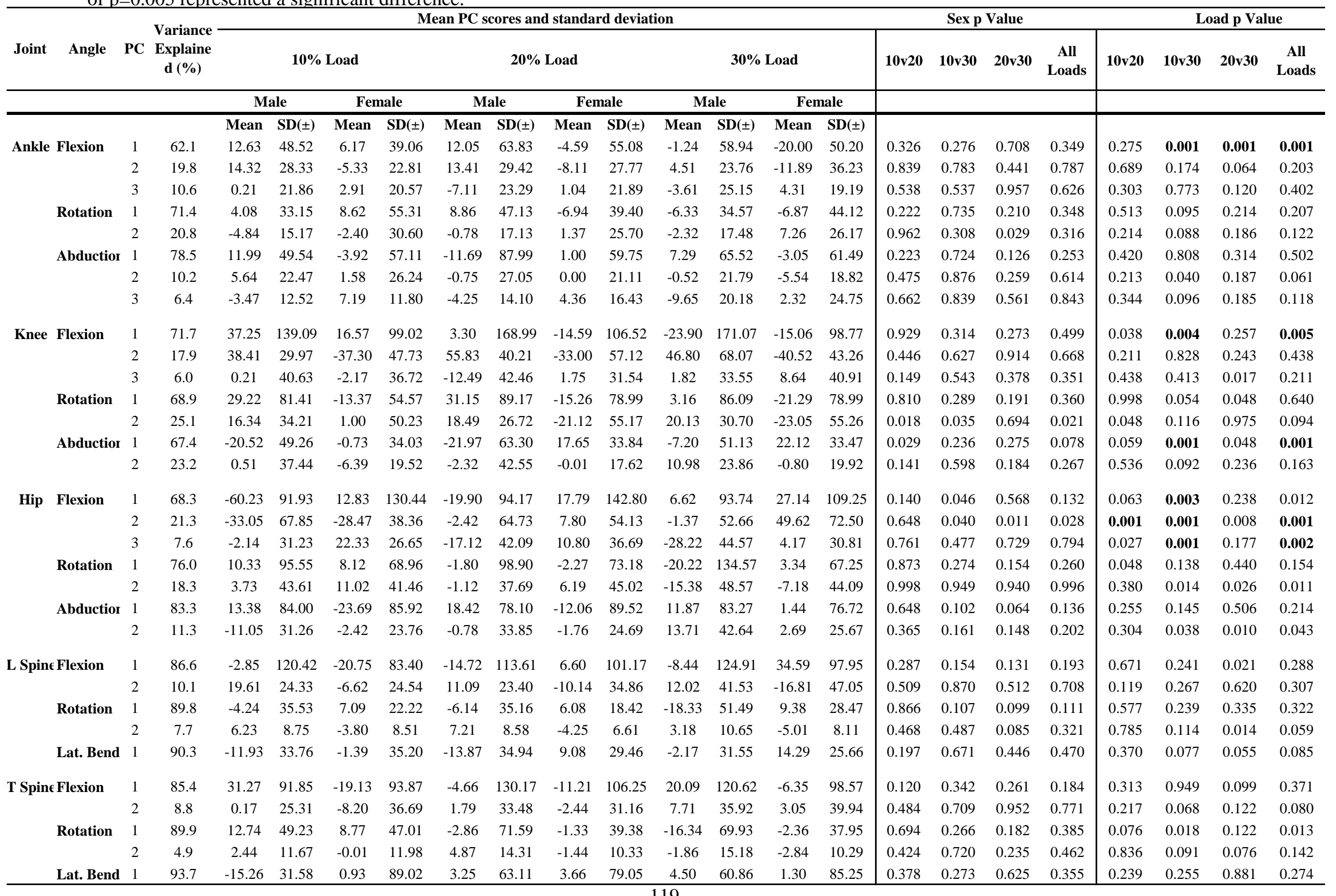




\section{Appendix G}

\section{Raw Curve Data}



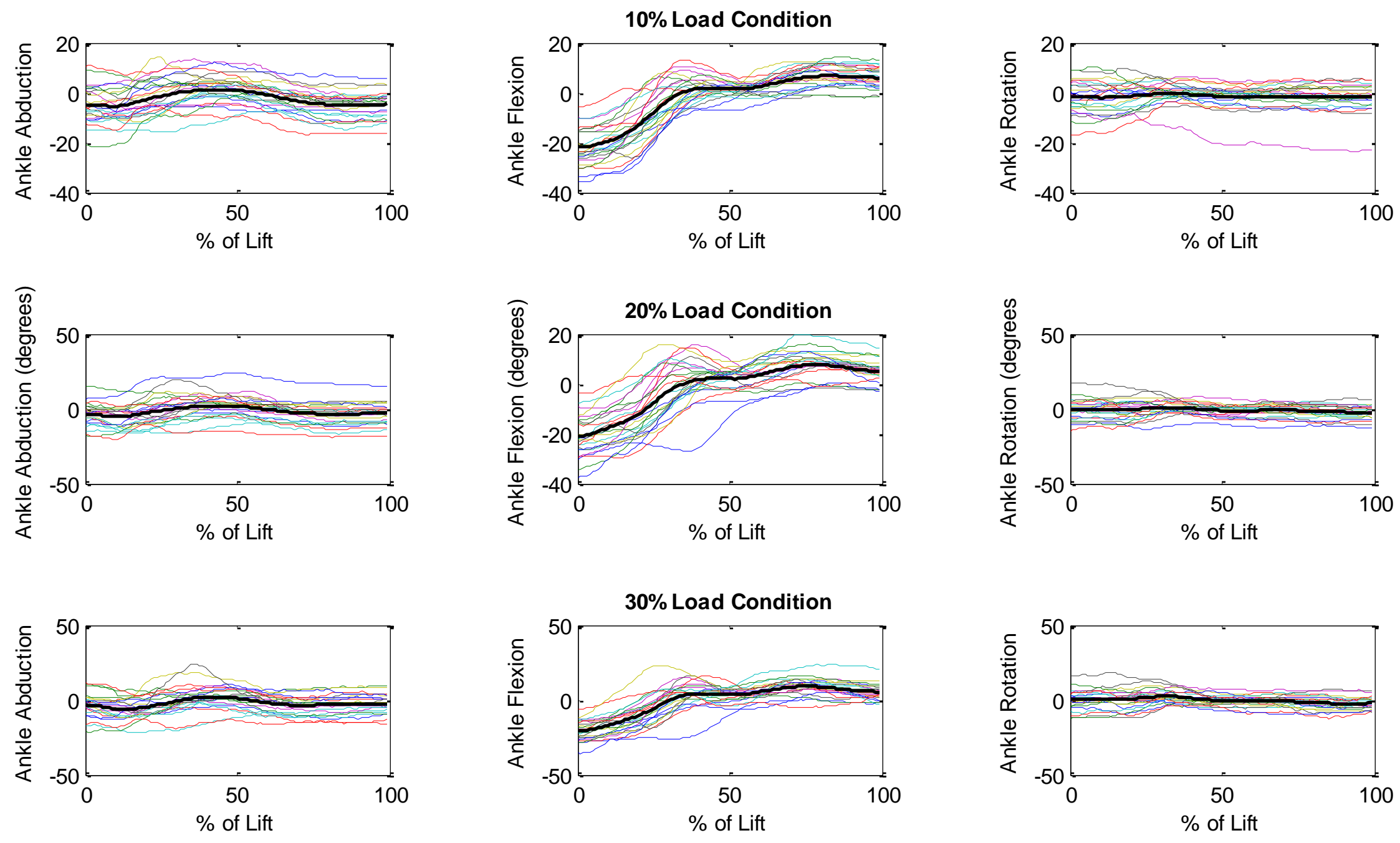

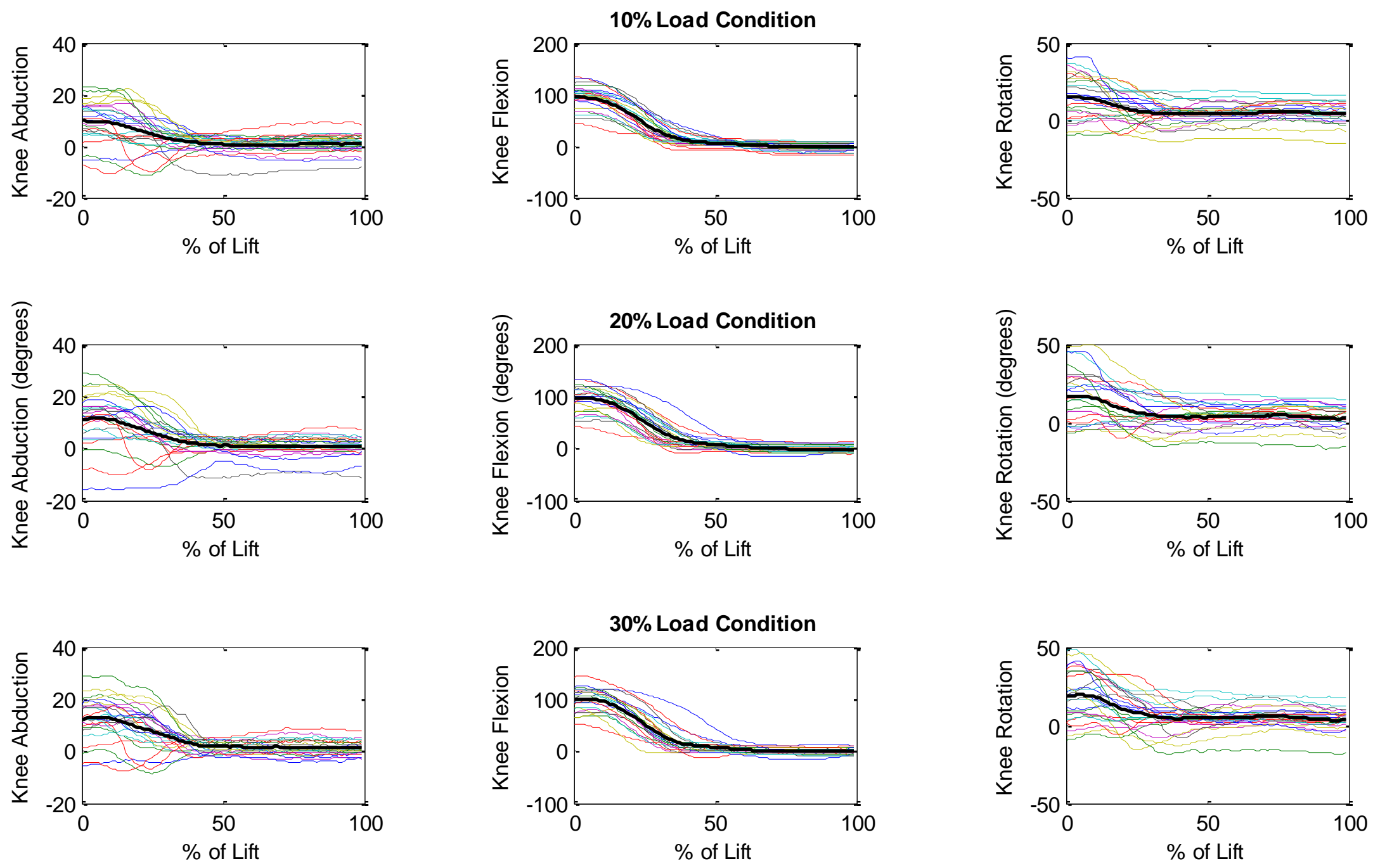

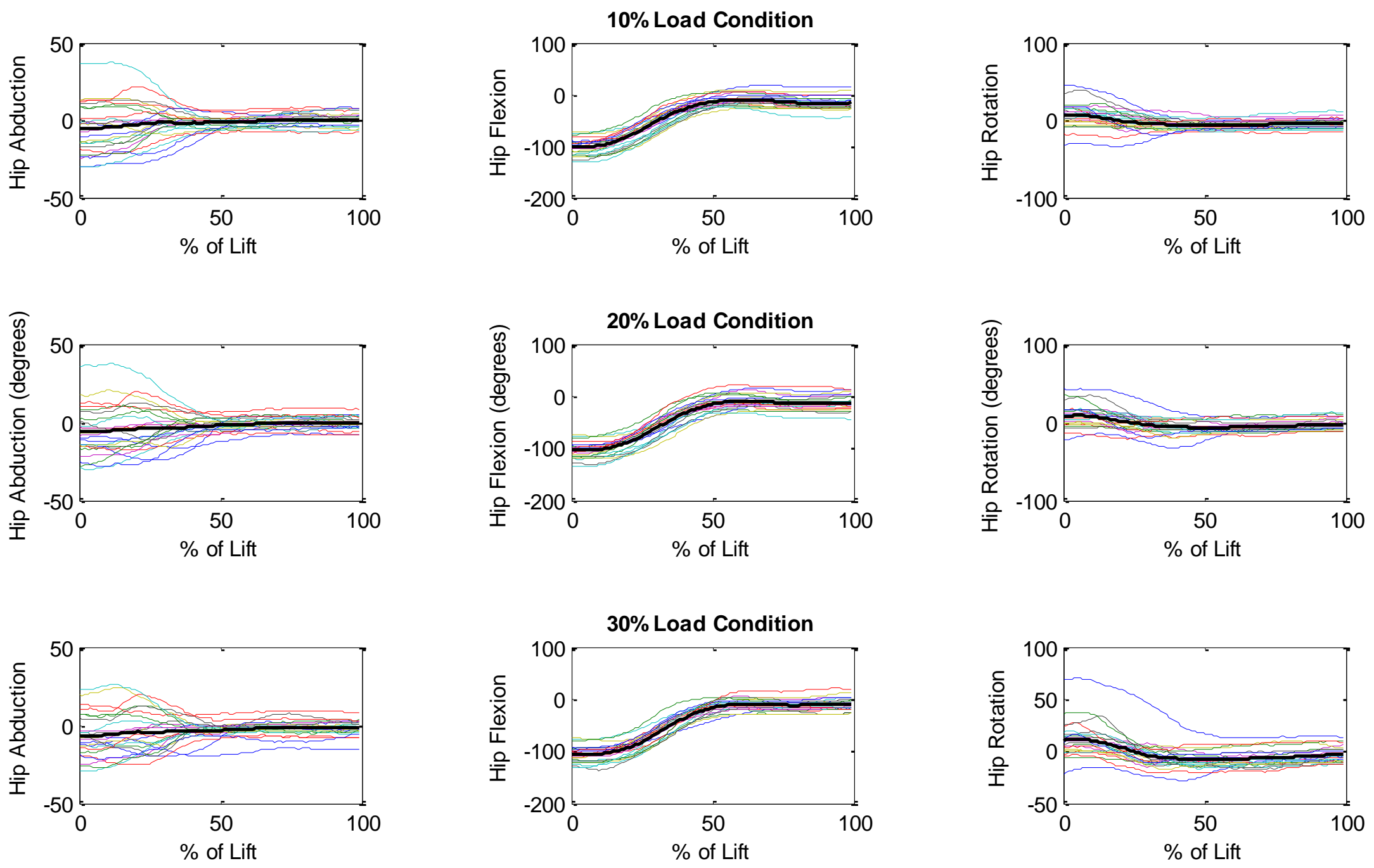

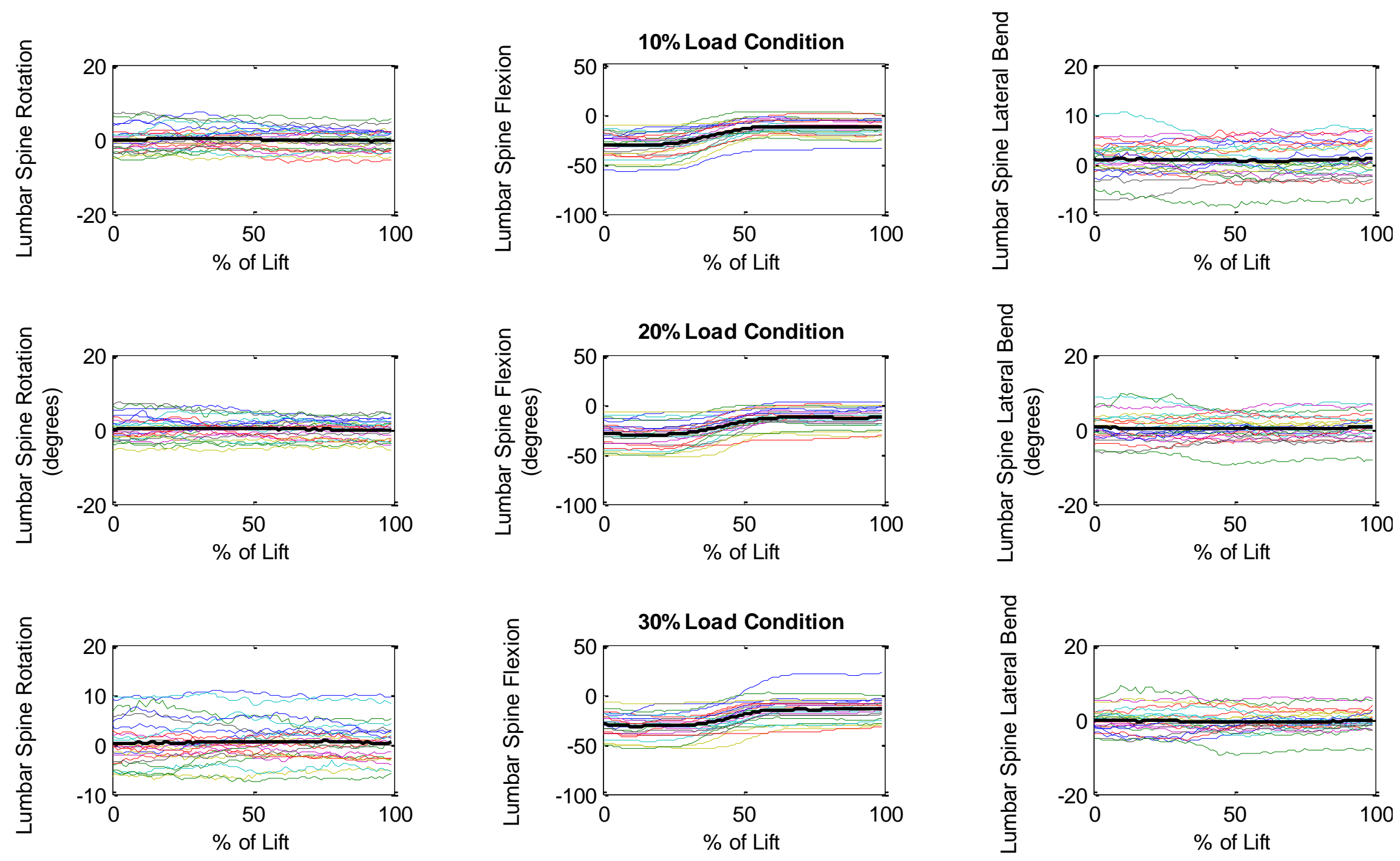

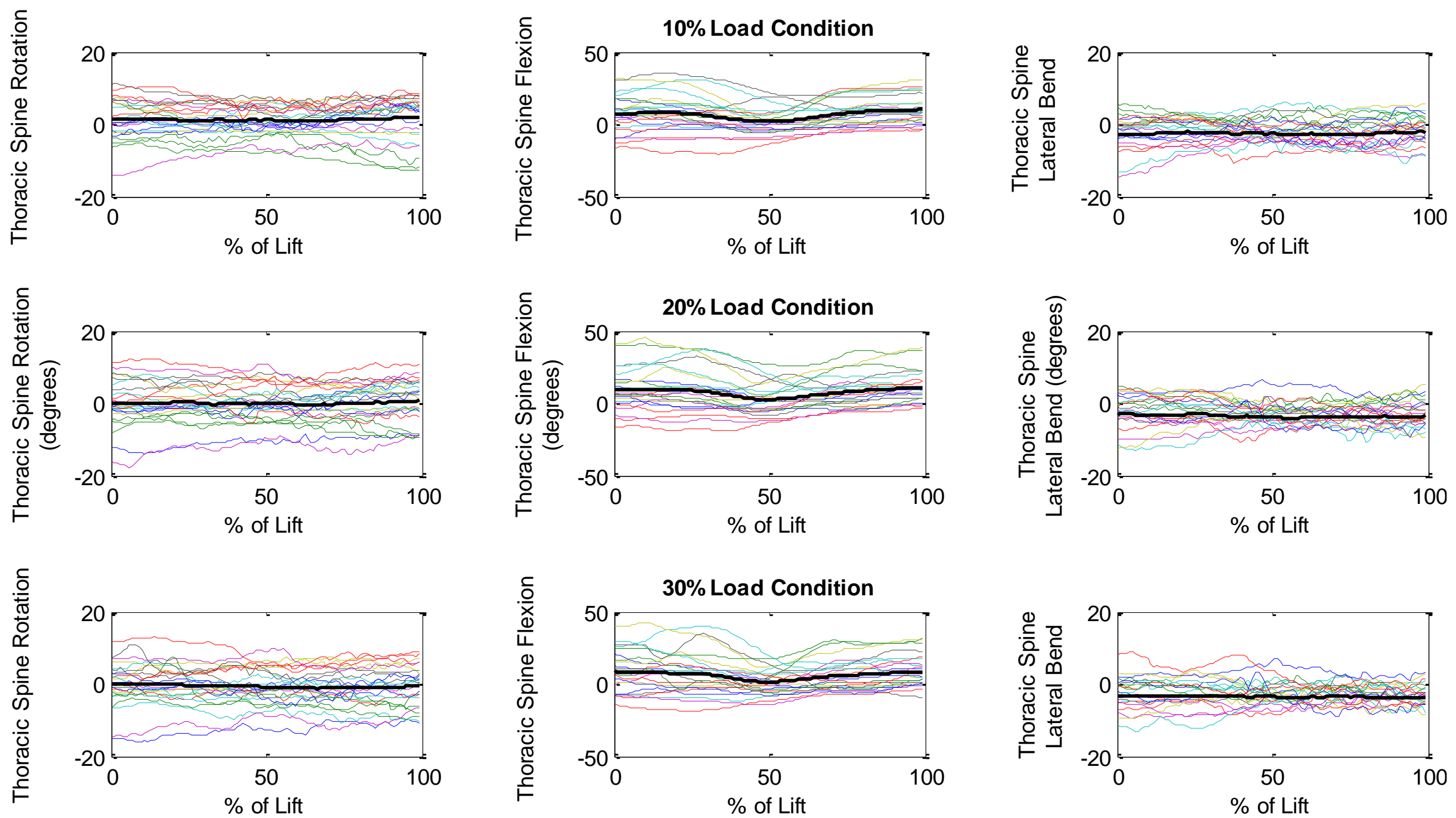